\title{
Developing a Modern Approach to Account for Steric Effects in Hammett-type Correlations
}

Celine B. Santiago, Anat Milo, and Matthew S. Sigman*

corresponding email: sigman@chem.utah.edu

\section{Supporting Information}

\section{Table of Contents}

$\begin{array}{ll}\text { Chemical Synthesis } & \\ \text { a) General considerations } & \text { S2 } \\ \text { b) General procedure and characterization data } & \text { S3 } \\ \text { DFT Calculations } & \text { S6 } \\ \text { Mathematical Modeling } & \text { S14 } \\ \text { Cartesian Coordinates of Geometry Optimized Structures } & \text { S25 } \\ \text { References } & \text { S91 } \\ \text { NMR Spectra } & \text { S92 }\end{array}$




\section{Chemical Synthesis}

General considerations

Dimethylformamide (DMF) was stored over activated $3 \AA$ molecular sieves. Ground $3 \AA$ MS were activated by heating at $200{ }^{\circ} \mathrm{C}$ for 20 min under $N_{2}$. Unless otherwise noted, all chemicals were purchased from Aldrich, Acros, TCl, or Combi and used without further purification. $\mathrm{Pd}\left(\mathrm{CH}_{3} \mathrm{CN}\right)_{2}(\mathrm{OTs})_{2}$ and ligand $L$ were synthesized according to literature procedure. ${ }^{1}{ }^{1} \mathrm{H}$ NMR spectra were obtained at 400 $\mathrm{MHz}$ or $500 \mathrm{MHz}$ using Varian spectrometers. ${ }^{1} \mathrm{H}$ chemical shifts are reported in ppm and referenced to $\mathrm{CDCl}_{3}$ at $7.26 \mathrm{ppm} .{ }^{13} \mathrm{C}$ NMR spectra were obtained at $75 \mathrm{MHz}, 100 \mathrm{MHz}$ or $125 \mathrm{MHz}$ using Varian spectrometers. ${ }^{13} \mathrm{C}$ chemical shifts are reported in ppm and referenced to $\mathrm{CDCl} 3$ at $77.16 \mathrm{ppm}$. Thin-layer chromatography (TLC) was performed with EMD silica gel 60 F254 plates, eluted with indicated solvents, visualized by a $254 \mathrm{~nm}$ UV lamp, and stained with phosphomolybdic acid. Flash chromatography was performed using EM reagent silica 60 (230-400 mesh). Infrared (IR) spectra were recorded using a Thermo Nicolet FT-IR. High resolution mass spectrometry (HRMS) data were obtained on a Waters LCP Premier XE instrument by ESI/TOF. GC separations were performed using Hewlett Packard HP 6890 series GC system fitted with an Agilent HP-5 column. Supercritical fluid chromatography (SFC) was performed at $40^{\circ} \mathrm{C}$, using a Thar instrument fitted with a chiral stationary phase as indicated. 
General procedure A: asymmetric Heck reaction

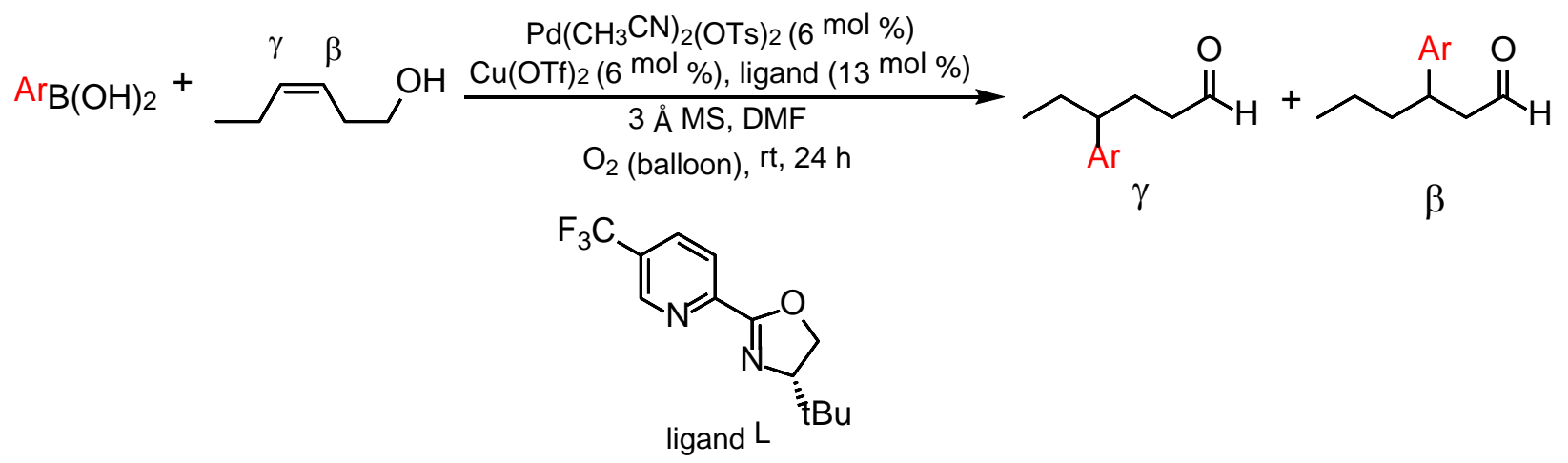

To a dry $100 \mathrm{~mL}$ Schlenk flask equipped with a stir bar, $\mathrm{Pd}\left(\mathrm{CH}_{3} \mathrm{CN}\right)_{2}(\mathrm{OTs})_{2}(15.9 \mathrm{mg}, 0.0300$ mmol, $6.00 \mathrm{~mol} \%), \mathrm{Cu}(\mathrm{OTf})_{2}$ (10.9 mg, $0.0300 \mathrm{mmol}, 6.00 \mathrm{~mol} \%$ ), ligand 1 (17.7 mg, $\left.0.0650 \mathrm{mmol}\right), 3 \AA$ MS (75 mg, $150 \mathrm{mg} / \mathrm{mmol})$, and DMF (8 mL) were added. A three-way adapter fitted with a balloon of $\mathrm{O}_{2}$ was added. The resulting mixture was stirred for $10 \mathrm{~min}$., before adding alkenyl alcohol $(0.5 \mathrm{mmol})$ in DMF (2 mL) and boronic acid (1.5 mmol, 3 equiv) with a syringe. After stirring for $24 \mathrm{~h}$ at room temperature, the mixture was transferred to a separatory funnel using diethyl ether $(100 \mathrm{~mL})$ and water $(50 \mathrm{~mL})$. The product was extracted into diethyl ether $(2 \times 50 \mathrm{~mL})$, washed with water $(1 \times 50 \mathrm{~mL})$ and brine $(1 \times 50 \mathrm{~mL})$, and dried over magnesium sulfate. The organic extracts were concentrated under reduced pressure, and the resulting residue was purified by silica gel flash chromatography, using 2-10\% EtOAc in hexane containing $0.1 \%$ triethylamine, to yield the aldehyde product.

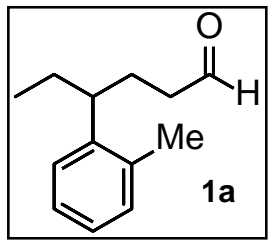

Compound 1a was prepared according to the general procedure A. The product 1a was isolated along with regioisomeric product (28:1). TLC (10\% EtOAc in hexane). Analytical data were found to match that of the literature. ${ }^{2}$

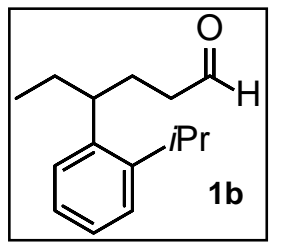

Compound $1 \mathrm{~b}$ was prepared according to the general procedure $\mathrm{A}$. The product $1 \mathrm{~b}$ was isolated in $21 \%$ yield along with regioisomeric product (24:1). TLC (10\% EtOAc in hexane). $\mathrm{R}_{f}=0.24 .[\alpha]^{20}{ }_{\mathrm{D}}=+4^{\circ}\left(\mathrm{c}=0.242, \mathrm{CHCl}_{3}\right) \cdot{ }^{1} \mathrm{HNMR}\left(500 \mathrm{MHz}, \mathrm{CDCl}_{3}\right) \delta=$ $9.67(\mathrm{t}, \mathrm{J}=1.5 \mathrm{~Hz}, 1 \mathrm{H}), 7.27(\mathrm{~m}, 1 \mathrm{H}), 7.18(\mathrm{~m}, 3 \mathrm{H}), 3.23(\mathrm{~m}, 1 \mathrm{H}), 2.93(\mathrm{~m}, 1 \mathrm{H}), 2.28(\mathrm{~m}, 2 \mathrm{H}), 2.06(\mathrm{~m}$, $1 \mathrm{H}), 1.86(\mathrm{~m}, 1 \mathrm{H}), 1.71(\mathrm{~m}, 1 \mathrm{H}), 1.62(\mathrm{~m}, 1 \mathrm{H}), 1.25(\mathrm{~m}, 7 \mathrm{H}), 0.82(\mathrm{t}, \mathrm{J}=7.5 \mathrm{~Hz}, 3 \mathrm{H}) .{ }^{13} \mathrm{C}-\mathrm{NMR}(125$ $\left.\mathrm{MHz}, \mathrm{CDCl}_{3}\right) \delta=202.7,147.3,141.2,126.4,126.2,125.4,42.4,30.2,28.7,28.2,24.5,24.3,12.4 . \mathrm{IR}$ 
(neat): $2960,2928,2871,1723,1488,1447,756 \mathrm{~cm}^{-1}$. HRMS (ESI-TOF) $\mathrm{m} / \mathrm{z}$ calcd for $\mathrm{C}_{15} \mathrm{H}_{22} \mathrm{ONa}$ $(\mathrm{M}+\mathrm{Na})^{+}: 241.1568$, found 241.1566 .

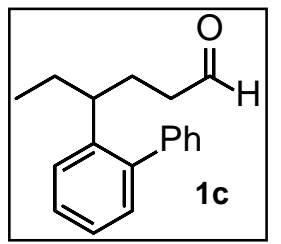

Compound $1 \mathrm{c}$ was prepared according to the general procedure $\mathrm{A}$. The product $1 \mathrm{c}$ was isolated in $62 \%$ yield along with regioisomeric product $(28: 1)$. TLC (10\% EtOAc in hexane). $\mathrm{R}_{f}=0.32 .[\alpha]_{\mathrm{D}}^{20}=+2^{\circ}\left(\mathrm{c}=0.222, \mathrm{CHCl}_{3}\right) .{ }^{1} \mathrm{H} \mathrm{NMR}\left(500 \mathrm{MHz}, \mathrm{CDCl}_{3}\right) \delta=$ $9.66(t, J=1.5 \mathrm{~Hz}, 1 \mathrm{H}), 7.33-7.43(\mathrm{~d}, 4 \mathrm{H}), 7.19-7.30(\mathrm{~d}, 5 \mathrm{H}), 2.70(\mathrm{~m}, 1 \mathrm{H}), 2.15(\mathrm{~m}, 2 \mathrm{H}), 1.90(\mathrm{~m}, 1 \mathrm{H})$, $1.77(\mathrm{~m}, 1 \mathrm{H}), 1.63(\mathrm{~m}, 2 \mathrm{H}), 0.77(\mathrm{t}, \mathrm{J}=7.5 \mathrm{~Hz}, 3 \mathrm{H}) .{ }^{13} \mathrm{C} \operatorname{NMR}\left(125 \mathrm{MHz}, \mathrm{CDCl}_{3}\right) \delta=202.6,143.2$, $142.3,142.1,130.1,129.8,129.6,128.2,128.1,127.0,125.9,125.8,125.7,42.3,41.2,30.1,29.2,12.4$. IR (neat): 2959, 2928, 2872, 2360, 2342, 1723, 1684, 1478, 1457, 1448, 1437, 755, 703, $668 \mathrm{~cm}^{-1}$. HRMS $(\mathrm{M}+\mathrm{Na})^{+}$calcd. 275.1412 obsvd. 275.1396. HRMS (ESI-TOF) $\mathrm{m} / \mathrm{z}$ calcd for $\mathrm{C}_{18} \mathrm{H}_{20} \mathrm{ONa}(\mathrm{M}+\mathrm{Na})^{+}$: 275.1412 , found 275.1396 .

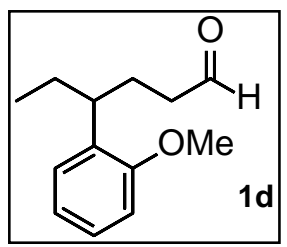

Compound $1 \mathrm{~d}$ was prepared according to the general procedure $A$. The product $1 \mathrm{~d}$ was isolated in $66 \%$ yield along with regioisomeric product (3.6:1). TLC (10\% EtOAc in hexane). $\mathrm{R}_{f}=0.39 \cdot[\alpha]_{\mathrm{D}}^{20}=+4^{\circ}\left(\mathrm{c}=0.208, \mathrm{CHCl}_{3}\right) \cdot{ }^{1} \mathrm{H} \mathrm{NMR}\left(500 \mathrm{MHz}, \mathrm{CDCl}_{3}\right) \delta=$ $9.65(t, J=2.0 \mathrm{~Hz}, 1 \mathrm{H}), 6.85-7.21(\mathrm{~m}, 6 \mathrm{H}), 3.78(\mathrm{~m}, 5 \mathrm{H}), 3.03(\mathrm{~m}, 1 \mathrm{H}), 2.67(\mathrm{~m}, 1 \mathrm{H}), 2.26(\mathrm{~m}, 2 \mathrm{H})$, $2.02(\mathrm{~m}, 1 \mathrm{H}), 1.83(\mathrm{~m}, 1 \mathrm{H}), 1.63(\mathrm{~m}, 3 \mathrm{H}), 1.22(\mathrm{~m}, 1 \mathrm{H}), 0.88(\mathrm{~m}, \mathrm{~J}=7.5 \mathrm{~Hz}, 1 \mathrm{H}), 0.81(\mathrm{t}, \mathrm{J}=7.5 \mathrm{~Hz}, 3$ H). ${ }^{13} \mathrm{C}$ NMR $\left(125 \mathrm{MHz}, \mathrm{CDCl}_{3}\right) \delta=203.2,158.0,131.7,127.7,127.2,120.9,120.6,110.8,55.6,42.3$, 28.5, 27.9, 12.3. IR (neat): 2957, 2933, 2360, 2342, 1718, 1506, 1491, 1464, 1457, 1240, $753 \mathrm{~cm}^{-1}$. HRMS (ESI-TOF) $\mathrm{m} / \mathrm{z}$ calcd for $\mathrm{C}_{13} \mathrm{H}_{18} \mathrm{ONa}(\mathrm{M}+\mathrm{Na})^{+}:$: 229.1204, found 229.1208.

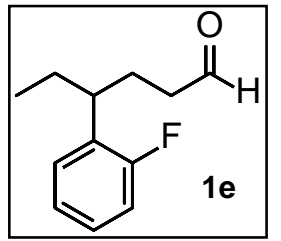

Compound $1 \mathrm{e}$ was prepared according to the general procedure A. The product $1 \mathrm{e}$ was isolated in $28 \%$ yield along with regioisomeric product $(30: 1)$. TLC (10\% EtOAc in hexane). $\mathrm{R}_{f}=0.30[\alpha]^{20}{ }_{\mathrm{D}}=-3^{\circ}\left(\mathrm{c}=0.228, \mathrm{CHCl}_{3}\right) .{ }^{1} \mathrm{H} \mathrm{NMR}\left(500 \mathrm{MHz}, \mathrm{CDCl}_{3}\right) \delta=$ $9.68(\mathrm{t}, \mathrm{J}=1.5 \mathrm{~Hz}, 1 \mathrm{H}), 7.15(\mathrm{~m}, 3 \mathrm{H}), 7.01(\mathrm{~m}, 1 \mathrm{H}), 2.86(\mathrm{~m}, 1 \mathrm{H}), 2.31(\mathrm{~m}, 2 \mathrm{H}), 2.05(\mathrm{~m}, 1 \mathrm{H}), 1.86(\mathrm{~m}$, $1 \mathrm{H}), 1.68(\mathrm{~m}, 2 \mathrm{H}), 0.83(\mathrm{~m}, \mathrm{~J}=6.5 \mathrm{~Hz}, 3 \mathrm{H}) .{ }^{13} \mathrm{C}$ NMR $\left(125 \mathrm{MHz}, \mathrm{CDCl}_{3}\right) \delta=202.5,128.7,127.9,127.8$, $124.5,115.7,115.5,42.3,39.9,28.7,27.7,12.3$. IR (neat): 2963, 2935, 2361, 2341, 2158, 1978, 1718, 
$1701,1490,1456,751,668 \mathrm{~cm}^{-1}$. HRMS (ESI-TOF) $\mathrm{m} / \mathrm{z}$ calcd for $\mathrm{C}_{12} \mathrm{H}_{15} \mathrm{FONa}(\mathrm{M}+\mathrm{Na})^{+}:$217.1005, found 217.1013.

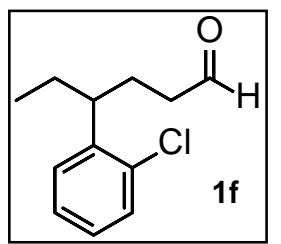

Compound $1 \mathrm{f}$ was prepared according to the general procedure A. The product $1 \mathrm{f}$ was isolated in 48\% yield along with regioisomeric product (100:1). TLC (10\% EtOAc in hexane). $\mathrm{R}_{f}=0.32 .[\alpha]^{20}=+5^{\circ}\left(\mathrm{c}=0.258, \mathrm{CHCl}_{3}\right) .{ }^{1} \mathrm{H} \mathrm{NMR}\left(500 \mathrm{MHz}, \mathrm{CDCl}_{3}\right) \delta=$ $9.68(\mathrm{~m}, \mathrm{~J}=1.5 \mathrm{~Hz}, 1 \mathrm{H}), 7.34\left(\mathrm{dd}, \mathrm{J}_{1}=6.0 \mathrm{~Hz}, J_{2}=1.5 \mathrm{~Hz}, 1 \mathrm{H}\right), 7.23(\mathrm{~m}, 1 \mathrm{H}), 7.18\left(\mathrm{dd}, \mathrm{J}_{1}=7.5 \mathrm{~Hz}, J_{2}\right.$ $=1.5 \mathrm{~Hz}, 1 \mathrm{H}), 7.13\left(\mathrm{dd}, \mathrm{J}_{1}=7.5 \mathrm{~Hz}, J_{2}=1.5 \mathrm{~Hz}, 1 \mathrm{H}\right), 3.19(\mathrm{~m}, 1 \mathrm{H}), 2.37(\mathrm{~m}, 1 \mathrm{H}), 2.24(\mathrm{~m}, 1 \mathrm{H}), 2.06$ $(\mathrm{m}, 1 \mathrm{H}), 1.82(\mathrm{~m}, 1 \mathrm{H}), 1.72(\mathrm{~m}, 1 \mathrm{H}), 1.62(\mathrm{~m}, 1 \mathrm{H}), 0.81(\mathrm{t}, \mathrm{J}=7.5 \mathrm{~Hz}, 3 \mathrm{H},) .{ }^{13} \mathrm{C}$ NMR $(125 \mathrm{MHz}$, $\left.\mathrm{CDCl}_{3}\right) \delta=202.4,141.9,135.0,129.8,127.8,127.5,127.4,42.0,30.0,29.2,28.2,12.0$. IR (neat): 2962, 2932, 2360, 2341, 1724, 1700, 1684, 1653, 1559, 1540, 1507, 1747, 1457, 1437, 755, $668 \mathrm{~cm}^{-1}$. HRMS (ESI-TOF) $\mathrm{m} / \mathrm{z}$ calcd for $\mathrm{C}_{12} \mathrm{H}_{15} \mathrm{ClONa}(\mathrm{M}+\mathrm{Na})^{+}:$233.0709, found 233.0711.

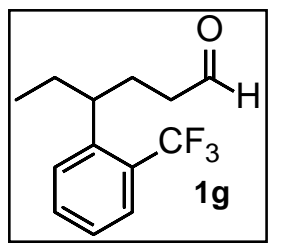

Compound $1 \mathrm{~g}$ was prepared according to the general procedure A. The product $1 \mathrm{~g}$ was isolated in 14\% yield along with regioisomeric product (65:1). TLC (10\% EtOAc in hexane $) \cdot \mathrm{R}_{f}=0.30 \cdot[\alpha]^{20}=+2^{\circ}\left(\mathrm{c}=0.194, \mathrm{CHCl}_{3}\right) \cdot{ }^{1} \mathrm{H} \mathrm{NMR}\left(500 \mathrm{MHz}, \mathrm{CDCl}_{3}\right) \delta=$ $9.66(\mathrm{~s}, 1 \mathrm{H}), 7.63(\mathrm{~m}, 1 \mathrm{H}), 7.52(\mathrm{~m}, 1 \mathrm{H}), 7.38(\mathrm{~m}, 1 \mathrm{H}), 7.27(\mathrm{~m}, 1 \mathrm{H}), 2.98(\mathrm{~m}, 1 \mathrm{H}), 2.39(\mathrm{~m}, 1 \mathrm{H}), 2.03-$ $2.20(\mathrm{~m}, 2 \mathrm{H}), 1.86(\mathrm{~m}, 1 \mathrm{H}), 1.72(\mathrm{~m}, 1 \mathrm{H}), 1.62(\mathrm{~m}, 1 \mathrm{H}), 0.79(\mathrm{t}, \mathrm{J}=7.5 \mathrm{~Hz}, 3 \mathrm{H}) .{ }^{13} \mathrm{C} \mathrm{NMR}(125 \mathrm{MHz}$, $\left.\mathrm{CDCl}_{3}\right) \delta=202.3,144.4,132.4,127.5,126.3,125.8,123.6,42.2,41.2,31.9,31.5,29.0,12.0 . \quad$ IR (neat): 2966, 2933, 1717, 1559, 1507, 1313, $1121 \mathrm{~cm}^{-1}$. HRMS (ESI-TOF) $\mathrm{m} / \mathrm{z}$ calcd for $\mathrm{C}_{13} \mathrm{H}_{15} \mathrm{~F}_{3} \mathrm{ONa}(\mathrm{M}+\mathrm{Na})^{+}$: 267.0973, found 267.0966.

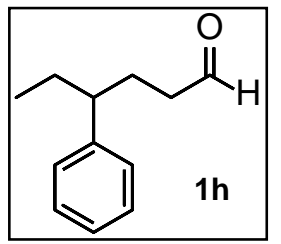

Compound $1 \mathrm{~h}$ was prepared according to the general procedure A. The product $1 \mathrm{~h}$ was isolated along with regioisomeric product (4.5:1). Analytical data were found to match that of the literature. ${ }^{2}$ 


\section{DFT Calculations}

Benzoic acids were geometrically optimized, with an ultrafine integration grid and ideal gas phase approximation, starting from a planar configuration of carbonyl to the benzene ring using Gaussian 09 software. ${ }^{3}$ DFT calculations of ground state structures were performed using M06-2x functional and a triple zeta potential basis set (jun-cc-pvtz), which was chosen based on the evaluation of the M06-2x functional for organic molecules with triple zeta quality basis sets resulting mostly to quantitative correlations. ${ }^{4}$ IR frequencies and intensities, NBO charges and torsional angles were obtained from the geometry optimized structures. Subsequently, Sterimol values of the substituent were calculated for the geometry optimized structures using Molecular Modeling Pro $\otimes^{5}$ Hammett values were taken from the compilation made by Hansch, Leo, and Taft. ${ }^{6}$

\section{Compilation of Parameters of Various Substituted Benzoic Acids}

Table S1. Sterimol and Hammett values of para-substituted benzoic acids.

\begin{tabular}{|c|c|c|c|c|}
\hline \multirow{2}{*}{$\begin{array}{l}\text { para } \\
\mathbf{R} \\
\end{array}$} & \multicolumn{3}{|c|}{ Sterimol } & \multirow{2}{*}{$\begin{array}{c}\text { Hammett } \\
\sigma_{, p}\end{array}$} \\
\hline & $B_{1, p}$ & $\mathbf{B}_{5, \mathrm{p}}$ & $\mathbf{L}_{p}$ & \\
\hline $4-\mathrm{H}$ & 1.00 & 1.00 & 2.09 & 0.00 \\
\hline $4-\mathrm{NMe}_{2}$ & 1.55 & 3.30 & 4.33 & -0.83 \\
\hline $4-\mathrm{NEt}_{2}$ & 2.58 & 3.80 & 5.29 & -0.72 \\
\hline 4- $\mathrm{NH}_{2}$ & 1.55 & 2.10 & 2.98 & -0.66 \\
\hline 4-Oi-Pr & 1.52 & 4.41 & 5.35 & -0.45 \\
\hline 4-OBn & 1.52 & 7.08 & 4.24 & -0.41 \\
\hline $4-\mathrm{OH}$ & 1.52 & 2.07 & 2.83 & -0.37 \\
\hline 4-OMe & 1.52 & 3.22 & 4.25 & -0.27 \\
\hline 4-OSiMe 3 & 1.52 & 4.90 & 5.95 & -0.27 \\
\hline 4-OEt & 1.52 & 3.22 & 5.25 & -0.24 \\
\hline 4-cyclopropyl & 1.77 & 3.42 & 4.32 & -0.21 \\
\hline $4-t-\mathrm{Bu}$ & 2.91 & 3.35 & 4.34 & -0.20 \\
\hline 4-Me & 1.70 & 2.20 & 3.07 & -0.17 \\
\hline $4-E t$ & 1.73 & 3.33 & 4.34 & -0.15 \\
\hline 4-i-Pr & 2.08 & 3.34 & 4.32 & -0.15 \\
\hline 4-cyclohexyl & 2.12 & 4.65 & 5.27 & -0.15 \\
\hline 4-cyclobutyl & 2.00 & 3.92 & 5.16 & -0.14 \\
\hline 4-cyclopentyl & 2.10 & 4.21 & 5.29 & -0.14 \\
\hline $4-B n$ & 1.72 & 5.95 & 5.13 & -0.09 \\
\hline $4-\mathrm{SiMe}_{3}$ & 3.10 & 3.66 & 4.79 & -0.07 \\
\hline 4-OPh & 1.52 & 5.43 & 5.43 & -0.03 \\
\hline 4-Ph & 1.77 & 3.16 & 6.36 & -0.01 \\
\hline
\end{tabular}




\begin{tabular}{l|lll|l|}
$4-\mathrm{F}$ & 1.47 & 1.47 & 2.80 & 0.06 \\
$4-\mathrm{N}_{3}$ & 1.60 & 1.60 & 4.11 & 0.08 \\
$4-\mathrm{Si}(\mathrm{OMe})_{3}$ & 2.69 & 4.20 & 5.40 & 0.13 \\
$4-\mathrm{Cl}$ & 1.77 & 1.77 & 3.47 & 0.23 \\
$4-\mathrm{Br}$ & 1.92 & 1.92 & 3.77 & 0.23 \\
$4-\mathrm{F}_{5} \mathrm{Ph}$ & 1.78 & 3.88 & 7.14 & 0.27 \\
$4-\mathrm{OAc}$ & 1.52 & 4.45 & 4.80 & 0.31 \\
$4-\mathrm{CHO}$ & 1.70 & 2.53 & 3.68 & 0.42 \\
$4-\mathrm{COPh}$ & 1.77 & 5.63 & 6.24 & 0.43 \\
$4-\mathrm{CO}_{2} \mathrm{Ph}$ & 1.70 & 5.28 & 8.12 & 0.44 \\
$4-\mathrm{CO}_{2} \mathrm{Me}$ & 1.78 & 3.60 & 5.08 & 0.45 \\
$4-\mathrm{CO}_{2} \mathrm{H}$ & 1.70 & 2.73 & 4.21 & 0.45 \\
$4-\mathrm{CCl}_{3}$ & 2.58 & 3.43 & 3.98 & 0.46 \\
$4-\mathrm{COMe}$ & 1.71 & 3.28 & 4.47 & 0.50 \\
$4-\mathrm{CF}_{3}$ & 2.08 & 2.71 & 3.58 & 0.54 \\
$4-\mathrm{C}\left(\mathrm{CF}_{3}\right)_{3}$ & 3.50 & 3.93 & 4.98 & 0.55 \\
$4-\mathrm{CN}$ & 1.78 & 1.78 & 4.18 & 0.66 \\
$4-\mathrm{SO}_{2} \mathrm{Ph}$ & 2.11 & 6.55 & 5.89 & 0.68 \\
$4-\mathrm{SO}_{2} \mathrm{Me}$ & 2.09 & 3.61 & 4.34 & 0.72 \\
$4-\mathrm{NO}_{2}$ & 1.55 & 2.58 & 3.61 & 0.78 \\
$4-\mathrm{COCF}_{3}$ & 1.86 & 3.76 & 5.00 & 0.80 \\
$4-\mathrm{NO}$ & 1.55 & 2.52 & 3.60 & 0.91 \\
$4-\mathrm{SO}_{2} \mathrm{~F}$ & 1.99 & 3.01 & 3.81 & 0.91 \\
$4-\mathrm{SO}_{2} \mathrm{CF}_{3}$ & 1.99 & 4.33 & 4.81 & 0.96 \\
$4-\mathrm{SO}_{2} \mathrm{Cl}$ & 2.00 & 3.84 & 3.81 & 1.11
\end{tabular}

Table S2. Infrared frequencies and intensities and carboxylic moiety NBO charges of para-substituted benzoic acids.

\begin{tabular}{|c|c|c|c|c|c|c|c|c|}
\hline \multirow{2}{*}{$\begin{array}{l}\text { para } \\
\mathbf{R}\end{array}$} & \multicolumn{4}{|c|}{ Infrared Vibrations } & \multicolumn{4}{|c|}{ NBO6 } \\
\hline & $\mathbf{v}_{\mathrm{COH}, \mathrm{p}}$ & $\mathbf{I}_{\mathrm{COH}, \mathrm{p}}$ & $\mathbf{v}_{\mathrm{C}=\mathrm{O}, \mathrm{p}}$ & $I_{C=0, p}$ & $\mathrm{NBO}_{\mathrm{c}, \mathrm{p}}$ & $\mathrm{NBO}_{=0, p}$ & $\mathrm{NBO}_{\mathrm{o}, \mathrm{p}}$ & $\mathrm{NBO}_{\mathrm{H}, \mathrm{p}}$ \\
\hline $4-\mathrm{H}$ & 1394.60 & 163.25 & 1847.85 & 390.68 & 0.80516 & -0.60208 & -0.70358 & 0.50087 \\
\hline 4-NMe ${ }_{2}$ & 396.43 & 313.71 & 1830.85 & 86.47 & 0.80471 & -0.62021 & -0.71045 & .49757 \\
\hline $4-\mathrm{NEt}_{2}$ & 1393.59 & 348.50 & 1828.93 & 502.08 & 0.80432 & -0.62180 & -0.71121 & 0.49726 \\
\hline $4-\mathrm{NH}_{2}$ & 1395.35 & 257.80 & 1834.97 & 453.68 & 0.80524 & -0.61626 & -0.70910 & 0.49865 \\
\hline 4-Oi-Pr & 1397.25 & 234.56 & 1837.65 & 435.72 & 0.80600 & -0.61295 & -0.70708 & 9950 \\
\hline 4-OBn & 1394.90 & 287.63 & 1839.44 & 414.29 & 0.80365 & -0.61160 & 7713 & 0.49929 \\
\hline $4-\mathrm{OH}$ & 1396.61 & 221.99 & & 22.67 & & & 602 & 016 \\
\hline & 1396.68 & & 1839.37 & 424.99 & 0.80480 & & 667 & 976 \\
\hline 4-OSiMe 3 & 1395.55 & 308.83 & 1837.85 & 428.26 & 0.80608 & -0.61247 & -0.70693 & 0.49958 \\
\hline 4-OEt & 1396.29 & 305.80 & 1838.90 & 431.63 & 0.80308 & -0.61298 & -0.70612 & 0.49940 \\
\hline 4-cycloprop) & 1394.35 & 196.10 & 1841.52 & 435.32 & 0.80433 & -0.60756 & -0.70549 & 0.49994 \\
\hline $4-t-B u$ & & 189.95 & 1844.67 & 435.29 & & & -0.70473 & 0.49999 \\
\hline 4-Me & 1395.02 & 178.02 & 1844.66 & 419.34 & 0.78588 & -0.60618 & -0.70599 & 0.50033 \\
\hline
\end{tabular}




\begin{tabular}{|c|c|c|c|c|c|c|c|c|}
\hline Et & 394.43 & 78.71 & 1843.43 & 425.97 & 0.80555 & -0.60560 & -0.70482 & \\
\hline$-i-\mathrm{Pr}$ & 395.08 & 176.22 & 1844.24 & 425.69 & 80588 & -0.60546 & -0.70491 & 019 \\
\hline -cyclohexyl & 393.74 & 145.83 & 1843.22 & 436.77 & 0.80592 & -0.60595 & 506 & 0010 \\
\hline -cyclobutyl & 395.35 & 62.58 & 842.99 & 37.23 & & & & \\
\hline$-\mathrm{cyc}$ & 395.12 & 88.60 & 1843.27 & 432.98 & 80591 & 0634 & 521 & \\
\hline 4-Bn & 395.43 & 85.02 & 1844.78 & 452.94 & & & & \\
\hline 4-SiMe ${ }_{3}$ & 394.24 & 169.33 & 1846.52 & 424.72 & 98 & & 362 & 047 \\
\hline 4-OPh & 395.54 & 305.76 & 1840.98 & 422.55 & .80416 & 916 & 45 & 50034 \\
\hline $\mathrm{Ph}$ & 394.96 & 228.79 & 1844.84 & 464.67 & & & & \\
\hline$-F$ & 396.19 & 204.97 & 1847.75 & 398.96 & 0.80562 & 0242 & 414 & 193 \\
\hline $4-N_{3}$ & 1396.87 & 305.63 & 1843.38 & 424.41 & 16 & & & 0.50170 \\
\hline 4-Si(C & 02 & 153.96 & 1847.74 & 410.30 & 68 & & -0.7 & \\
\hline$-\mathrm{Cl}$ & 395.38 & 186.22 & 1849.16 & 414.28 & 0.80492 & 26 & 85 & .50238 \\
\hline$-B r$ & .33 & 182.92 & 1849.58 & 419.46 & & & & \\
\hline$-F_{5} \mathrm{Ph}$ & 397.09 & 221.65 & 1850.24 & 430.32 & 0.80306 & 615 & 212 & 268 \\
\hline$-O A C$ & 395.43 & 217.52 & 1848.50 & 432.51 & 39 & & -0 & 285 \\
\hline $4-\mathrm{Cr}$ & & & & 218.69 & & & & \\
\hline $4-\mathrm{CC}$ & 397.17 & 145.45 & 1850.01 & 379.17 & & & & 248 \\
\hline $4-\mathrm{CO}$ & 1396.77 & 108.39 & 1848.47 & 666.94 & 272 & & -0.7 & 0296 \\
\hline $4-\mathrm{CO}$ & 1397.72 & 92.93 & 1851.64 & 297.43 & 0.80291 & & -0.7 & 0254 \\
\hline $4-\mathrm{CO}_{2} \mathrm{H}$ & 1388.15 & 314.30 & 1849.62 & 787.31 & 184 & 454 & 128 & 316 \\
\hline $4-\mathrm{CCl}_{3}$ & 1398.33 & 181.26 & 1852.88 & 408.71 & 0.80401 & 432 & -0.70087 & 0.50312 \\
\hline $4-\mathrm{CO}$ & .86 & 58.79 & 185 & 341.23 & & & & \\
\hline $4-\mathrm{CF}_{3}$ & 1401.11 & 45.48 & 1854.66 & 375.38 & 0.78303 & 324 & -0.7 & 0390 \\
\hline $4-\mathrm{C}\left(\mathrm{CF}_{3}\right)_{3}$ & 1402.66 & 170.16 & 1855.75 & 390.57 & 0.80384 & & -0.6 & \\
\hline $4-\mathrm{CN}$ & 1397.32 & 181.63 & 1855.52 & 393.01 & 0.80177 & & & 0474 \\
\hline $4-\mathrm{SO}_{2} \mathrm{Ph}$ & 1395.78 & 163.92 & 1854.33 & 396.00 & 0.80194 & 195 & 993 & 0.50357 \\
\hline & 1395.82 & 154.99 & 1855.62 & 383.71 & 30266 & & & 0444 \\
\hline $4-\mathrm{NO}_{2}$ & 1396.61 & 202.70 & 1857.56 & 370.43 & 0.78101 & -0.58788 & -0.69977 & 0.50541 \\
\hline $4-\mathrm{COCF}_{3}$ & 1400.65 & 183.12 & 1853.93 & 564.23 & 0.80101 & -0.58821 & -0.69867 & 0.50485 \\
\hline $4-\mathrm{NO}$ & 1397.34 & 157.39 & 1853.50 & 379.56 & 0.78020 & -0.59013 & -0.70083 & 0.50450 \\
\hline $4-\mathrm{SO}_{2} \mathrm{~F}$ & 1397.51 & 147.51 & 1859.62 & 367.40 & & -0.58436 & & 0.50617 \\
\hline & 1396.64 & 145.62 & 1859.09 & 369.14 & 0.80068 & -0.58372 & -0.69733 & 0.50618 \\
\hline $4-\mathrm{SO}_{2} \mathrm{Cl}$ & 1396.90 & 157.81 & 1858.22 & 376.92 & 0.80136 & -0.58502 & -0.69788 & 0.50599 \\
\hline
\end{tabular}

Table S3. Sterimol and Hammett values of meta-substituted benzoic acids.

\begin{tabular}{l|ccc|c|} 
& \multicolumn{3}{|c|}{ Sterimol } & Hammett \\
$\mathbf{R}$ & $\mathbf{B}_{\mathbf{1 , m}}$ & $\mathbf{B}_{5, \mathbf{m}}$ & $\mathbf{L}_{\mathbf{m}}$ & $\mathbf{\sigma}_{\mathbf{m} \mathbf{m}}$ \\
\hline 3-H & 1.00 & 1.00 & 2.09 & 0.00 \\
$3-\mathrm{NMe}_{2}$ & 2.00 & 3.28 & 4.11 & -0.16 \\
$3-\mathrm{NEt}_{2}$ & 1.98 & 4.60 & 4.05 & -0.23 \\
$3-\mathrm{NH}_{2}$ & 1.55 & 2.08 & 2.99 & -0.16 \\
3-Oi-Pr & 1.52 & 4.20 & 4.33 & 0.10
\end{tabular}




\begin{tabular}{|c|c|c|c|c|}
\hline 3-OBn & 1.52 & 7.08 & 4.24 & N/A \\
\hline 3-OH & 1.52 & 2.07 & 2.86 & 0.12 \\
\hline 3-OMe & 1.52 & 3.20 & 4.25 & 0.12 \\
\hline 3-OSiMe 3 & 1.52 & 4.84 & 5.91 & 0.13 \\
\hline 3-OEt & 1.52 & 3.20 & 5.24 & 0.10 \\
\hline 3-cyclopropyl & 1.75 & 3.38 & 4.32 & -0.07 \\
\hline $3-t-\mathrm{Bu}$ & 2.92 & 3.35 & 4.36 & -0.10 \\
\hline 3-Me & 1.70 & 2.19 & 3.07 & -0.07 \\
\hline 3-Et & 1.71 & 3.33 & 4.33 & -0.07 \\
\hline $3-i-\operatorname{Pr}$ & 2.08 & 3.34 & 4.33 & -0.04 \\
\hline 3-cyclohexyl & 2.11 & 3.60 & 6.44 & -0.05 \\
\hline 3-cyclobutyl & 1.93 & 3.98 & 4.81 & -0.05 \\
\hline 3-cyclopentyl & 2.08 & 3.31 & 5.38 & -0.05 \\
\hline 3-Bn & 1.72 & 5.94 & 5.29 & -0.08 \\
\hline 3-SiMe 3 & 3.09 & 3.65 & 4.76 & -0.04 \\
\hline 3-OPh & 1.52 & 5.48 & 5.83 & 0.25 \\
\hline 3-Ph & 1.77 & 3.14 & 6.36 & 0.06 \\
\hline $3-F$ & 1.47 & 1.47 & 2.81 & 0.34 \\
\hline $3-N_{3}$ & 1.55 & 3.66 & 4.14 & 0.37 \\
\hline $3-\mathrm{Si}(\mathrm{OMe})_{3}$ & 3.07 & 4.33 & 5.66 & 0.09 \\
\hline $3-\mathrm{Cl}$ & 1.77 & 1.77 & 3.51 & 0.37 \\
\hline $3-\mathrm{Br}$ & 1.92 & 1.92 & 3.81 & 0.39 \\
\hline $3-\mathrm{F}_{5} \mathrm{Ph}$ & 1.78 & 3.83 & 7.09 & 0.26 \\
\hline 3-OAc & 1.52 & 4.45 & 4.80 & 0.39 \\
\hline $3-\mathrm{CHO}$ & 1.70 & 2.51 & 3.68 & 0.35 \\
\hline 3-COPh & 1.78 & 5.63 & 6.22 & 0.34 \\
\hline $3-\mathrm{CO}_{2} \mathrm{Ph}$ & 1.88 & 4.61 & 8.13 & 0.37 \\
\hline $3-\mathrm{CO}_{2} \mathrm{Me}$ & 1.80 & 3.52 & 5.00 & 0.37 \\
\hline $3-\mathrm{CO}_{2} \mathrm{H}$ & 1.70 & 2.76 & 4.14 & 0.37 \\
\hline $3-\mathrm{CCl}_{3}$ & 2.59 & 3.45 & 3.99 & 0.40 \\
\hline 3-COMe & 1.70 & 3.27 & 4.46 & 0.38 \\
\hline $3-\mathrm{CF}_{3}$ & 2.09 & 2.72 & 3.48 & 0.43 \\
\hline $3-\mathrm{C}\left(\mathrm{CF}_{3}\right)_{3}$ & 3.42 & 3.88 & 4.92 & 0.55 \\
\hline $3-\mathrm{CN}$ & 1.78 & 1.78 & 4.18 & 0.56 \\
\hline $3-\mathrm{SO}_{2} \mathrm{Ph}$ & 2.09 & 6.46 & 4.08 & 0.62 \\
\hline $3-\mathrm{SO}_{2} \mathrm{Me}$ & 2.08 & 3.53 & 4.46 & 0.60 \\
\hline $3-\mathrm{NO}_{2}$ & 1.55 & 2.59 & 3.56 & 0.71 \\
\hline $3-\mathrm{COCF}_{3}$ & 1.84 & 3.82 & 4.99 & 0.63 \\
\hline 3-NO & 1.55 & 2.61 & 3.47 & 0.62 \\
\hline $3-\mathrm{SO}_{2} \mathrm{~F}$ & 2.00 & 3.03 & 3.78 & 0.80 \\
\hline $3-\mathrm{SO}_{2} \mathrm{CF}_{3}$ & 2.02 & 4.09 & 4.92 & 0.83 \\
\hline $3-\mathrm{SO}_{2} \mathrm{Cl}$ & 2.03 & 3.76 & 3.77 & 1.20 \\
\hline
\end{tabular}


Table S4. Infrared frequencies and intensities and carboxylic moiety NBO charges of meta-substituted benzoic acids.

\begin{tabular}{|c|c|c|c|c|c|c|c|c|}
\hline \multirow{2}{*}{$\begin{array}{l}\text { meta } \\
\mathrm{R}\end{array}$} & \multicolumn{4}{|c|}{ Infrared Vibrations } & \multicolumn{4}{|c|}{ NBO6 } \\
\hline & $\mathbf{v}_{\mathrm{COH}, \mathrm{m}}$ & $\mathrm{I}_{\mathrm{COH}, \mathrm{m}}$ & $\mathbf{V}_{\mathrm{C}=\mathrm{O}, \mathrm{m}}$ & $I_{C=0, m}$ & $\mathrm{NBO}_{\mathrm{c}, \mathrm{m}}$ & $\mathrm{NBO}_{=0, \mathrm{~m}}$ & $\mathrm{NBO}_{\mathrm{o}, \mathrm{m}}$ & $\mathrm{NBO}_{\mathrm{H}, \mathrm{m}}$ \\
\hline 3-H & 394.60 & 163.25 & 1847.85 & 390.68 & 0.80516 & -0.60208 & -0.70358 & 0.50087 \\
\hline 3-NMe ${ }_{2}$ & 1378.42 & 106.94 & 1845.61 & 398.56 & 0.80655 & -0.60340 & -0.70782 & 0.49914 \\
\hline 3-NEt ${ }_{2}$ & 1387.04 & 136.13 & 1845.14 & 411.75 & 0.80698 & -0.60371 & -0.70869 & 0.49908 \\
\hline 3- $\mathrm{NH}_{2}$ & 1398.20 & 172.81 & 1847.74 & 379.29 & 0.80639 & -0.60132 & -0.70622 & 0.49971 \\
\hline 3-Oi-Pr & 1394.18 & 191.60 & 1847.12 & 398.35 & 0.80646 & -0.60198 & -0.70247 & 0.49965 \\
\hline 3-OBn & 1393.04 & 234.11 & 1848.30 & 406.68 & 0.80491 & 0095 & -0.70269 & 0.49989 \\
\hline $3-\mathrm{OH}$ & 398.66 & 189.34 & 1849.36 & 370.78 & 0.80529 & -0.59959 & -0.70183 & 0.50059 \\
\hline 3-OMe & 396.67 & 216.86 & 1847.51 & 385.34 & 0.80599 & & -0.70218 & 0.50019 \\
\hline $3-\mathrm{OS}$ & 1395.85 & 192.40 & 1847.13 & 406.56 & 0.80407 & -0.60140 & 0446 & 0.50011 \\
\hline 3-OEt & 1395.51 & 209.46 & 1847.78 & 392.76 & 0.80500 & -0.60109 & 0299 & 0.49991 \\
\hline 3-cy & 1389 & 107. & 1846 & 413.67 & 44 & & 514 & 981 \\
\hline $3-t-B u$ & 1395.11 & 148.49 & 1845.19 & 426.62 & 0.80583 & -0.60299 & 0606 & 0.50005 \\
\hline 3-Me & 1395.49 & 157.59 & 1846.08 & 400.44 & 0.80602 & & 452 & 996 \\
\hline 3-Et & 397.22 & 155.62 & 1846.68 & 409.88 & 0.80598 & -0.6 & 460 & 990 \\
\hline $3-i-\operatorname{Pr}$ & 1399.36 & 135.69 & 1846.23 & 417.31 & 0.80503 & 301 & 579 & 996 \\
\hline & & & & & & & & 966 \\
\hline 3-cy & 1398.21 & 163.39 & 1846.29 & 422.16 & 0.80536 & & 505 & 979 \\
\hline 3-сус & 398.77 & 127.46 & 1844.76 & 429.42 & 515 & & 522 & 969 \\
\hline 3-Bn & 1398.22 & & & 426.07 & 431 & & 234 & 038 \\
\hline 3-SiMe 3 & 1394.90 & 158.82 & 1845.55 & 436.08 & 0.80505 & 341 & 530 & 005 \\
\hline & 396.21 & 245.8 & & 25 & & & & 079 \\
\hline 3-Ph & 1398.75 & 166.61 & 1847.33 & 441.95 & 0.80492 & & 404 & 060 \\
\hline $3-F$ & 397.94 & 186.91 & 1852.75 & 369.96 & 0.80394 & 554 & 171 & 0.50225 \\
\hline $3-N_{3}$ & & & 1852.08 & 386.85 & & & & 260 \\
\hline 3-Si(C & 1396.77 & 168.80 & 1844.92 & 435.89 & 0.80353 & -0.60499 & 182 & 019 \\
\hline $3-\mathrm{Cl}$ & 1394.82 & & & 389.42 & 0.80547 & & 118 & 252 \\
\hline $3-\mathrm{Br}$ & & & & 396.40 & & & & 265 \\
\hline $3-\mathrm{F}_{5} \mathrm{Ph}$ & 1401.76 & 173.09 & 1851.33 & 432.30 & 0.80351 & -0.59661 & -0.70195 & 0.50238 \\
\hline & 1397.04 & & & & 0.80257 & & 70242 & 0.50317 \\
\hline & 1400.31 & 170.14 & 1851.32 & 353.74 & 0.80489 & -0.59721 & -0.69660 & 0.50265 \\
\hline & 1400.99 & 152.39 & 1849.05 & 434.74 & 0.80398 & -0.60003 & 9817 & 0.50156 \\
\hline & 1402.89 & 244.86 & 1851.89 & 170.02 & 0.80404 & -0.59845 & -0.70059 & 0.50255 \\
\hline $3-\mathrm{CO}_{2} \mathrm{Me}$ & 1404.11 & 250.16 & 1850.19 & 355.31 & 0.80428 & -0.59917 & -0.70153 & 0.50178 \\
\hline & 1407.92 & 92.75 & 1850.56 & 408.70 & 0.80443 & -0.59861 & -0.69790 & 0.50223 \\
\hline & 1399.92 & 166.43 & 1851.92 & 418.87 & 0.80502 & -0.59529 & -0.70030 & 0.50306 \\
\hline 3-COMe & 1404.08 & 207.03 & 1851.35 & 374.51 & 0.80435 & -0.59719 & -0.70520 & 0.50240 \\
\hline & 1408.57 & 201.35 & 1853.23 & 388.18 & 0.80364 & -0.59415 & -0.70028 & 0.50352 \\
\hline $3-\mathrm{C}\left(\mathrm{CF}_{3}\right)_{3}$ & 1400.36 & 165.09 & 1853.31 & 407.31 & 0.80489 & -0.59382 & -0.70008 & 0.50408 \\
\hline $3-\mathrm{CN}$ & 1401.10 & 182.03 & 1856.38 & 395.52 & 0.80490 & -0.59114 & -0.69879 & 0.50472 \\
\hline
\end{tabular}




\begin{tabular}{|c|c|c|c|c|c|c|c|c|}
\hline $\mathrm{SO}_{2} \mathrm{Ph}$ & 397.92 & 182.69 & 1852.35 & 423.43 & 0.80268 & -0.59493 & -0.69883 & 0.50288 \\
\hline $\mathrm{SO}_{2} \mathrm{Me}$ & 399.38 & 191.29 & 1853.66 & 405.52 & 0.80352 & -0.59328 & -0.69805 & 399 \\
\hline$-\mathrm{NO}_{2}$ & 399.96 & 179.66 & 1856.20 & 387.73 & 0.80328 & -0.59 & -0.6 & 490 \\
\hline$-\mathrm{COCF}_{3}$ & 1381.24 & 78.67 & 1852.76 & 584.81 & 0.80382 & -0.59340 & -0.69948 & 0.50465 \\
\hline $\mathrm{NO}$ & 1399.71 & 175.92 & 1852.88 & 390.51 & 0.80456 & -0.59414 & -0.69849 & 0.50363 \\
\hline$-\mathrm{SO}_{2} \mathrm{~F}$ & 1400.85 & 161.98 & 1857.30 & 391.80 & 0.80314 & -0.58849 & -0.69634 & 0.50592 \\
\hline c0 & 1399.88 & 54.25 & 1857.18 & 400.75 & 0.80271 & -0.58877 & -0.69609 & 0.50583 \\
\hline$-\mathrm{SO}_{2} \mathrm{Cl}$ & 1400.31 & 149.54 & 1856.88 & 400.75 & 0.80312 & -0.58877 & -0.69636 & 0.50578 \\
\hline
\end{tabular}

Table S5. Sterimol values, Hammett values, and torsion angles of ortho-substituted benzoic acids.

\begin{tabular}{|c|c|c|c|c|}
\hline \multirow{2}{*}{$\begin{array}{l}\text { ortho } \\
\mathbf{R}\end{array}$} & \multicolumn{3}{|c|}{ Sterimol } & \multirow{2}{*}{$\begin{array}{c}\text { Torsion Angle } \\
\theta_{\text {tor }}\end{array}$} \\
\hline & $\mathbf{B}_{1,0}$ & $B_{5,0}$ & $\mathbf{L}_{0}$ & \\
\hline $2-\mathrm{H}$ & 1.00 & 1.00 & 2.09 & 0.00 \\
\hline $2-\mathrm{NMe}_{2}$ & 1.55 & 3.27 & 4.30 & 25.77 \\
\hline $2-\mathrm{NEt}_{2}$ & 2.58 & 3.80 & 5.29 & 54.39 \\
\hline 2- $\mathrm{NH}_{2}$ & 1.55 & 2.06 & 3.03 & 2.60 \\
\hline 2-Oi-Pr & 1.52 & 4.41 & 5.35 & 17.73 \\
\hline 2-OBn & 1.52 & 4.50 & 8.23 & 7.62 \\
\hline $2-\mathrm{OH}$ & 1.52 & 2.07 & 2.83 & 0.00 \\
\hline 2-OMe & 1.52 & 3.22 & 4.25 & 21.20 \\
\hline $2-\mathrm{OSiMe}_{3}$ & 1.52 & 4.90 & 5.95 & 9.96 \\
\hline 2-OEt & 1.52 & 3.22 & 5.25 & 13.34 \\
\hline 2-cyclopropyl & 1.74 & 3.39 & 4.32 & 27.47 \\
\hline $2-t-\mathrm{Bu}$ & 2.91 & 3.35 & 4.34 & 56.76 \\
\hline 2-Me & 1.70 & 2.20 & 3.07 & 0.00 \\
\hline $2-E t$ & 1.73 & 3.33 & 4.34 & 17.45 \\
\hline $2-i-\mathrm{Pr}$ & 2.08 & 3.34 & 4.32 & 28.60 \\
\hline 2-cyclohexyl & 2.12 & 3.63 & 6.47 & 29.32 \\
\hline 2-cyclobutyl & 2.06 & 3.42 & 5.40 & 41.23 \\
\hline 2-cyclopentyl & 2.07 & 3.40 & 5.44 & 29.73 \\
\hline 2-Bn & 1.74 & 5.82 & 5.57 & 26.31 \\
\hline $2-\mathrm{SiMe}_{3}$ & 2.95 & 3.67 & 4.86 & 17.54 \\
\hline 2-OPh & 1.52 & 5.94 & 5.72 & 24.05 \\
\hline 2-Ph & 1.77 & 3.16 & 6.36 & 35.70 \\
\hline $2-\mathrm{F}$ & 1.47 & 1.47 & 2.80 & 0.00 \\
\hline $2-\mathrm{N}_{3}$ & 1.55 & 3.60 & 4.24 & 23.65 \\
\hline $2-\mathrm{Si}(\mathrm{OMe})_{3}$ & 2.84 & 4.45 & 5.67 & 18.60 \\
\hline $2-\mathrm{Cl}$ & 1.77 & 1.77 & 3.47 & 25.80 \\
\hline $2-\mathrm{Br}$ & 1.92 & 1.92 & 3.77 & 30.30 \\
\hline $2-\mathrm{F}_{5} \mathrm{Ph}$ & 1.77 & 3.83 & 7.08 & 21.52 \\
\hline 2-OAC & 1.52 & 4.61 & 4.55 & 18.93 \\
\hline $2-\mathrm{CHO}$ & 1.70 & 2.53 & 3.68 & 21.95 \\
\hline 2-COPh & 1.70 & 5.66 & 6.38 & 26.73 \\
\hline
\end{tabular}




\begin{tabular}{l|ccc|c|}
$2-\mathrm{CO}_{2} \mathrm{Ph}$ & 1.83 & 4.63 & 8.02 & 32.34 \\
$2-\mathrm{CO}_{2} \mathrm{Me}$ & 1.78 & 3.60 & 5.08 & 33.08 \\
$2-\mathrm{CO}_{2} \mathrm{H}$ & 1.70 & 2.73 & 4.21 & 34.82 \\
$2-\mathrm{CCl}_{3}$ & 2.58 & 3.45 & 3.97 & 49.24 \\
$2-\mathrm{COMe}_{2}$ & 1.71 & 3.28 & 4.47 & 17.95 \\
$2-\mathrm{CF}_{3}$ & 2.08 & 2.71 & 3.58 & 36.60 \\
$\left.2-\mathrm{C} \mathrm{CF}_{3}\right)_{3}$ & 3.31 & 3.88 & 4.93 & 44.19 \\
$2-\mathrm{CN}$ & 1.78 & 1.78 & 4.18 & 29.19 \\
$2-\mathrm{SO}_{2} \mathrm{Ph}$ & 2.11 & 6.34 & 5.19 & 43.75 \\
$2-\mathrm{SO}_{2} \mathrm{Me}$ & 2.11 & 3.53 & 4.51 & 47.83 \\
$2-\mathrm{NO}_{2}$ & 1.55 & 2.58 & 3.61 & 44.26 \\
$2-\mathrm{COCF}_{3}$ & 1.73 & 3.88 & 4.76 & 14.72 \\
$2-\mathrm{NO}$ & 1.55 & 2.61 & 3.47 & 34.66 \\
$2-\mathrm{SO}_{2} \mathrm{~F}$ & 1.98 & 3.04 & 3.84 & 35.67 \\
$2-\mathrm{SO}_{2} \mathrm{CF}_{3}$ & 2.02 & 2.9 & 3.54 & 38.96 \\
$2-\mathrm{SO}_{2} \mathrm{Cl}$ & 2.02 & 3.78 & 3.81 & 45.91
\end{tabular}

Table S6. Infrared frequencies and intensities and carboxylic moiety NBO charges of ortho-substituted benzoic acids.

\begin{tabular}{|c|c|c|c|c|c|c|c|c|}
\hline \multirow{2}{*}{$\begin{array}{l}\text { ortho } \\
\mathbf{R}\end{array}$} & \multicolumn{4}{|c|}{ Infared Vibrations } & \multicolumn{4}{|c|}{ NBO6 } \\
\hline & $\mathbf{v}_{\mathrm{COH}, \mathrm{o}}$ & $\mathrm{I}_{\mathrm{COH}, \mathrm{O}}$ & $\mathbf{V}_{\mathrm{C}=\mathrm{O}, \mathrm{o}}$ & $I_{C=0,0}$ & $\mathrm{NBO}_{\mathrm{C}, \mathrm{o}}$ & $\mathrm{NBO}_{=0,0}$ & $\mathrm{NBO}_{\mathrm{O}, \mathrm{o}}$ & $\mathrm{NBO}_{\mathrm{H}, \mathrm{O}}$ \\
\hline $2-\mathrm{H}$ & 1394.60 & 163.25 & 1847.85 & 390.68 & 0.80516 & -0.60208 & -0.70358 & 0.50087 \\
\hline $2-\mathrm{NMe}_{2}$ & 1385.34 & 46.99 & 1826.81 & 455.50 & 0.80697 & -0.61279 & -0.69946 & 0.49588 \\
\hline $2-\mathrm{NEt}_{2}$ & 1395.45 & 110.58 & 1845.04 & 368.11 & 0.82279 & -0.60042 & -0.68793 & 0.49537 \\
\hline $2-\mathrm{NH}_{2}$ & 1388.97 & 76.63 & 1830.53 & 481.33 & 0.80849 & -0.61276 & -0.73355 & 0.50600 \\
\hline 2-Oi-Pr & 1405.74 & 124.40 & 1823.01 & 461.85 & 0.81074 & -0.61663 & -0.68248 & 0.49519 \\
\hline $2-\mathrm{OBn}$ & 1406.17 & 141.86 & 1823.59 & 501.16 & 0.81017 & -0.61454 & -0.69008 & 0.49723 \\
\hline $2-\mathrm{OH}$ & 1408.63 & 122.13 & 1825.36 & 430.86 & 0.80852 & -0.61647 & -0.68045 & 0.49706 \\
\hline 2-OMe & 1399.72 & 131.89 & 1837.11 & 429.50 & 0.81217 & -0.60468 & -0.69401 & 0.49943 \\
\hline $2-\mathrm{OSiMe}_{3}$ & 1399.39 & 127.99 & 1828.96 & 474.74 & 0.81031 & -0.61062 & -0.69756 & 0.49869 \\
\hline 2-OEt & 1406.97 & 132.59 & 1822.23 & 454.93 & 0.80968 & -0.61753 & -0.68188 & 0.49530 \\
\hline 2-cyclopropyl & 1390.15 & 107.38 & 1835.92 & 436.65 & 0.81044 & -0.60479 & -0.70387 & 0.49988 \\
\hline $2-t-B u$ & 1378.50 & 99.39 & 1852.54 & 355.57 & 0.82059 & -0.59468 & -0.70647 & 0.49794 \\
\hline 2-Me & 1391.63 & 122.69 & 1831.67 & 408.67 & 0.80753 & -0.61086 & -0.70423 & 0.50162 \\
\hline 2-Et & 1389.01 & 106.29 & 1834.10 & 421.41 & 0.80914 & -0.60726 & -0.70699 & 0.50198 \\
\hline $2-i-\mathrm{Pr}$ & 1394.74 & 67.91 & 1834.99 & 386.61 & 0.81044 & -0.60389 & -0.70609 & 0.50054 \\
\hline 2-cyclohexyl & 1390.92 & 65.79 & 1838.41 & 439.95 & 0.81090 & -0.60302 & -0.70604 & 0.50063 \\
\hline 2-cyclobutyl & 1384.90 & 100.25 & 1842.94 & 381.12 & 0.81268 & -0.59770 & -0.70773 & 0.49968 \\
\hline
\end{tabular}




\begin{tabular}{|c|c|c|c|c|c|c|c|c|}
\hline 2-cyclop & 388.03 & 102.61 & 1839.18 & 436.20 & 0.81057 & -0.60276 & -0.70512 & 0.50013 \\
\hline 2-Bn & 1391.16 & 112.90 & 1840.42 & 348.09 & 0.81170 & -0.60037 & -0.70169 & 0.50212 \\
\hline$-\mathrm{SiMe}_{3}$ & 1385.28 & 131.13 & 1842.05 & 423.41 & 0.80747 & -0.60228 & -0.70672 & 0.50453 \\
\hline OPh & 1401.72 & 143.08 & 1838.96 & 369.21 & 0.81071 & -0.59973 & -0.68696 & 0.50106 \\
\hline $\mathrm{Ph}$ & 1392.06 & 116.36 & 1841.06 & 405.59 & 0.80929 & -0.60133 & -0.68967 & 0.49861 \\
\hline$F$ & 1407.80 & 155.11 & 1837.86 & 410.90 & 0.80809 & -0.60458 & -0.68210 & 0.49971 \\
\hline $2-N_{3}$ & 1386.84 & 44.09 & 1845.33 & 406.50 & 0.80388 & -0.59393 & -0.70359 & 0.50441 \\
\hline$-\mathrm{Si}(\mathrm{OMe})_{3}$ & 1387.60 & 161.03 & 1851.59 & 430.15 & 0.81093 & -0.59775 & -0.70837 & 0.50580 \\
\hline$-\mathrm{Cl}$ & 1403.56 & 136.98 & 1838.98 & 404.79 & 0.81116 & -0.59957 & -0.68514 & 0.50130 \\
\hline$-\mathrm{Br}$ & 1401.92 & 130.52 & 1842.55 & 407.66 & 0.81188 & -0.59658 & -0.68619 & 0.50127 \\
\hline $2-\mathrm{F}_{5} \mathrm{Ph}$ & 1392.48 & 127.35 & 1849.98 & 408.30 & 0.80785 & -0.59171 & -0.70323 & 0.50385 \\
\hline -OAC & 1395.67 & 183.71 & 1845.88 & 465.06 & 0.80902 & -0.59380 & -0.69873 & 0.50694 \\
\hline$-\mathrm{CHO}$ & 1387.67 & 129.92 & 1830.31 & 274.48 & 0.80307 & -0.59086 & -0.70210 & 0.50469 \\
\hline$-\mathrm{COPh}$ & 1391.29 & 152.38 & 1849.35 & 336.34 & 0.80714 & -0.59377 & -0.69305 & 0.50420 \\
\hline $2-\mathrm{CO}_{2} \mathrm{Ph}$ & 1389.45 & 201.65 & 1856.27 & 625.85 & 0.81325 & -0.58752 & -0.70069 & 0.50348 \\
\hline $2-\mathrm{CO}_{2} \mathrm{Me}$ & 1390.98 & 193.02 & 1841.27 & 411.85 & 0.81412 & -0.59127 & -0.69719 & 0.50017 \\
\hline $2-\mathrm{CO}_{2} \mathrm{H}$ & 1399.71 & 248.55 & 1856.05 & 756.68 & 0.81381 & -0.58901 & -0.69530 & 0.50101 \\
\hline $2-\mathrm{CCl}_{3}$ & 1392.45 & 117.78 & 1857.24 & 357.25 & 0.81610 & -0.58877 & -0.69340 & 0.50160 \\
\hline 2-COMe & 1387.01 & 157.23 & 1850.01 & 357.29 & 0.80431 & -0.59330 & -0.69946 & 0.50491 \\
\hline $2-\mathrm{CF}_{3}$ & 1402.74 & 230.78 & 1854.51 & 371.41 & 0.80925 & -0.58821 & -0.68729 & 0.50302 \\
\hline $2-\mathrm{C}\left(\mathrm{CF}_{3}\right)_{3}$ & 1397.27 & 186.57 & 1860.18 & 382.40 & 0.81964 & -0.59264 & -0.69455 & 0.50406 \\
\hline $2-\mathrm{CN}$ & 1403.17 & 160.60 & 1847.07 & 386.94 & 0.80262 & -0.59353 & -0.68333 & 0.50810 \\
\hline $2-\mathrm{SO}_{2} \mathrm{Ph}$ & 1396.85 & 157.12 & 1865.53 & 424.96 & 0.81801 & -0.57866 & -0.69515 & 0.50349 \\
\hline $2-\mathrm{SO}_{2} \mathrm{Me}$ & 1393.28 & 149.80 & 1871.81 & 371.67 & 0.82285 & -0.57255 & -0.70793 & 0.50635 \\
\hline $2-\mathrm{NO}_{2}$ & 1395.67 & 112.67 & 1869.31 & 375.43 & 0.81941 & -0.57682 & -0.69015 & 0.50473 \\
\hline $2-\mathrm{COCF}_{3}$ & & 142.56 & & 434.60 & 0.80949 & -0.58046 & & 0.51196 \\
\hline 2-NO & 1400.90 & 147.94 & 1850.99 & 387.61 & 0.80724 & -0.58934 & -0.67988 & 0.50173 \\
\hline $2-\mathrm{SO}_{2} \mathrm{~F}$ & 1397.13 & 169.19 & 1867.56 & 381.86 & 0.81072 & -0.57427 & -0.69038 & 0.50965 \\
\hline $2-\mathrm{SO}_{2} \mathrm{CF}_{3}$ & 1405.68 & 304.42 & 1872.06 & 385.14 & 0.81446 & -0.57083 & -0.70128 & 0.51082 \\
\hline$-\mathrm{SO}_{2} \mathrm{Cl}$ & 1392.97 & 142.47 & 1873.24 & 381.64 & 0.81505 & -0.57151 & -0.69019 & 0.50756 \\
\hline
\end{tabular}




\section{Mathematical Modeling}

Using MATLAB student version R2014a ${ }^{7}$, a script used to develop multivariate linear regression models was edited from a literature procedure. ${ }^{8}$ Parameters used in the model were normalized using the formula: $X_{\text {norm }}=(X-\mu) / \sigma$, where $X_{\text {norm }}$ is the normalized parameter, $X$ is the parameter, $\mu$ is the mean, and $\sigma$ is the standard deviation.

According to Curtin-Hammett principle, the relative rate of formation of competing products $(X$ and $Y)$ is logarithmically related to the difference in transition state energies, represented by the measured $\Delta \Delta \mathrm{G}^{\ddagger}$ (equation 1 ), where $\mathrm{R}$ is the gas constant and $\mathrm{T}$ is temperature. To derive measured $\Delta \Delta \mathrm{G}^{\ddagger}$ values, product ratios resulting from differences in selectivity were obtained experimentally. Parameters that describe the steric and electronic perturbations induced by the substituents on the ring were then identified. Using stepwise linear regression in Matlab ${ }^{\circ},{ }^{7}$ a mathematical model was developed relating the identified steric and electronic parameters, represented by $A, B$, and $C$, to the predicted $\Delta \Delta G^{\ddagger}$ (equation 2). ${ }^{9}$ Thus, the predicted $\Delta \Delta G^{\ddagger}$ values can be compared with the measured $\Delta \Delta G^{\ddagger}$ values obtained from experimental results. This correlation is possible in cases where all of the compared product ratios are the result of a similar mechanism of product determination. A good correlation between the two indicates that the structural parameters included in the equation may adequately approximate the effects leading to the measured $\Delta \Delta \mathrm{G}^{\ddagger}$.

$$
\begin{aligned}
& \text { measured } \Delta \Delta G^{\ddagger}=-R T \ln \left(\frac{X}{Y}\right) \\
& \text { predicted } \Delta \Delta G^{\ddagger}=\alpha A+\beta B+\gamma C
\end{aligned}
$$

\section{A. Linear Regression Models for Palladium-catalyzed redox-relay Heck Reaction}

Table S7. Parameters, measured and predicted transition state energies, $\Delta \Delta G^{\ddagger}$, of redox relay Heck

\begin{tabular}{|c|c|c|c|c|c|c|c|c|}
\hline $\mathbf{R}$ & Ratio & $E_{s}$ & $\mathbf{F}$ & $\sigma_{\text {para }}$ & Ratio & $\underset{\Delta \mathbf{G}^{\ddagger}}{\text { Measured }^{\mathrm{a}} \boldsymbol{\Delta}}$ & $\begin{array}{c}\text { Predicted } \\
\Delta \mathbf{G}^{\ddagger}\end{array}$ & $\begin{array}{c}\text { LOO } \\
\text { Predicted }^{\text {a }} \boldsymbol{\Delta} \\
\boldsymbol{\Delta G}^{\ddagger}\end{array}$ \\
\hline 2-Me & 30.0 & -1.24 & 0.01 & -0.17 & 30.0 & 2.01 & 1.68 & 1.51 \\
\hline $2-\mathrm{CF}_{3}$ & 64.5 & -2.40 & 0.38 & 0.54 & 64.5 & 2.47 & 2.94 & 4.97 \\
\hline 2-OMe & 4.5 & -0.55 & 0.29 & -0.27 & 4.5 & 0.89 & 1.52 & 2.32 \\
\hline
\end{tabular}
reaction ortho model developed using the Fujita Equation (data for Figure 5). 


\begin{tabular}{ccccccccc}
$2-\mathrm{F}$ & 29.5 & -0.46 & 0.45 & 0.06 & 29.5 & 2.00 & 1.84 & 1.70 \\
$2-\mathrm{Cl}$ & 100 & -0.97 & 0.42 & 0.23 & 100 & 2.73 & 2.15 & 1.89 \\
$2-i-\mathrm{Pr}$ & 24 & -1.71 & 0.04 & -0.15 & 24 & 1.88 & 1.91 & 1.94 \\
$2-\mathrm{Ph}$ & 46.5 & -1.01 & 0.12 & -0.01 & 46.5 & 2.27 & 1.78 & 1.68 \\
$\mathrm{H}$ & 4.45 & 0.00 & 0.03 & 0.00 & 4.45 & 0.88 & 1.31 & 3.70 \\
\hline
\end{tabular}

${ }^{\mathrm{a}} \Delta \Delta \mathrm{G}^{\mp}$ is reported in $\mathrm{kcal} / \mathrm{mol}$.

Table S8. Calculated and literature-derived parameters for redox relay Heck reaction ortho model.

\begin{tabular}{c|ccc|cc}
\hline & \multicolumn{3}{|c|}{ Sterimol } & \multicolumn{2}{c}{ Hammett } \\
$\mathbf{R}$ & $\mathbf{B}_{1, \mathbf{o}}$ & $\mathbf{B}_{5, \mathbf{o}}$ & $\mathbf{L}_{\mathbf{o}}$ & $\boldsymbol{\sigma}_{\mathrm{m}}$ & $\boldsymbol{\sigma}_{\mathbf{p}}$ \\
\hline $2-\mathrm{Me}$ & 1.70 & 2.20 & 3.07 & -0.07 & -0.17 \\
$2-\mathrm{CF}_{3}$ & 2.08 & 2.71 & 3.58 & 0.43 & 0.54 \\
$2-\mathrm{OMe}$ & 1.52 & 3.22 & 4.25 & 0.12 & -0.27 \\
$2-\mathrm{F}$ & 1.47 & 1.47 & 2.80 & 0.34 & 0.06 \\
$2-\mathrm{Cl}$ & 1.77 & 1.77 & 3.47 & 0.37 & 0.23 \\
$2-i-\mathrm{Pr}$ & 2.08 & 3.34 & 4.32 & -0.04 & -0.15 \\
$2-\mathrm{Ph}$ & 1.77 & 3.16 & 6.36 & 0.06 & -0.01 \\
$\mathrm{H}$ & 1.00 & 1.00 & 2.09 & 0.00 & 0.00 \\
\hline
\end{tabular}

Table S9. Infrared frequencies and intensities and carboxylic moiety NBO charges of ortho-substituted benzoic acids.

\begin{tabular}{|c|c|c|c|c|c|c|c|c|c|}
\hline \multirow[b]{2}{*}{$\mathbf{R}$} & \multicolumn{4}{|c|}{ IR Vibrations ortho } & \multicolumn{4}{|c|}{ NBO6 ortho } & \multirow[b]{2}{*}{$\boldsymbol{\theta}_{\text {tor }}$} \\
\hline & $\mathbf{v}_{\mathrm{COH}, \mathrm{O}}$ & $\mathrm{I}_{\mathrm{COH}, \mathrm{O}}$ & $\mathbf{V}_{\mathrm{C}=0,0}$ & $I_{C=0,0}$ & $\mathrm{NBO}_{\mathrm{C}, \mathrm{o}}$ & $\mathrm{NBO}_{=0,0}$ & $\mathrm{NBO}_{\mathrm{O}, \mathrm{o}}$ & $\mathrm{NBO}_{\mathrm{H}, \mathrm{O}}$ & \\
\hline 2-Me & 1391.63 & 122.69 & 1831.67 & 408.67 & 0.80753 & -0.61086 & -0.70423 & 0.50162 & 180.00 \\
\hline $2-\mathrm{CF}_{3}$ & 1402.74 & 230.78 & 1854.51 & 371.41 & 0.80925 & -0.58821 & -0.68729 & 0.50302 & 143.40 \\
\hline 2-OMe & 1399.72 & 131.89 & 1837.11 & 429.50 & 0.81217 & -0.60468 & -0.69401 & 0.49943 & 158.80 \\
\hline $2-\mathrm{F}$ & 1407.80 & 155.11 & 1837.86 & 410.90 & 0.80809 & -0.60458 & -0.68210 & 0.49971 & 180.00 \\
\hline $2-\mathrm{Cl}$ & 1403.56 & 136.98 & 1838.98 & 404.79 & 0.81116 & -0.59957 & -0.68514 & 0.50130 & 154.20 \\
\hline $2-i-\mathrm{Pr}$ & 1394.74 & 67.91 & 1834.99 & 386.61 & 0.81044 & -0.60389 & -0.70609 & 0.50054 & 151.40 \\
\hline $2-\mathrm{Ph}$ & 1392.06 & 116.36 & 1841.06 & 405.59 & 0.80929 & -0.60133 & -0.68967 & 0.49861 & 144.30 \\
\hline $\mathrm{H}$ & 1394.60 & 163.25 & 1847.85 & 390.68 & 0.80516 & -0.60208 & -0.70358 & 0.50087 & 180.00 \\
\hline
\end{tabular}

${ }^{\mathrm{D}} \mathrm{IR}$ frequency $(\mathrm{v})$ is reported in $\mathrm{cm}^{-1}$.

Table S10. Infrared frequencies and intensities and carboxylic moiety NBO charges of para-substituted benzoic acids. 


\begin{tabular}{c|cc|cccc}
$\mathbf{R}$ & $\mathbf{v}_{\mathbf{C}=\mathbf{0}, \mathbf{p}}$ & $\mathbf{I}_{\mathbf{C}=\mathbf{0}, \mathbf{p}}$ & $\mathbf{N B O}_{\mathbf{C}, \mathbf{p}}$ & $\mathbf{N B O}_{=\mathbf{0}, \mathbf{p}}$ & $\mathbf{N B O}_{\mathbf{0}, \mathbf{p}}$ & $\mathbf{N B O}_{\mathbf{H}, \mathbf{p}}$ \\
\hline $4-\mathrm{Me}$ & 1844.66 & 419.34 & 0.78588 & -0.60618 & -0.70599 & 0.50033 \\
$4-\mathrm{CF}_{3}$ & 1854.66 & 375.38 & 0.78303 & -0.59324 & -0.70152 & 0.50390 \\
$4-\mathrm{OMe}$ & 1839.37 & 424.99 & 0.80480 & -0.61182 & -0.70667 & 0.49976 \\
$4-\mathrm{F}$ & 1847.75 & 398.96 & 0.80562 & -0.60242 & -0.70414 & 0.50193 \\
$4-\mathrm{Cl}$ & 1849.16 & 414.28 & 0.80492 & -0.59926 & -0.70285 & 0.50238 \\
$4-i-\mathrm{Pr}$ & 1844.24 & 425.69 & 0.80588 & -0.60546 & -0.70491 & 0.50019 \\
$4-\mathrm{Ph}$ & 1844.84 & 464.67 & 0.80436 & -0.60323 & -0.70420 & 0.50067 \\
$\mathrm{H}$ & 1847.85 & 390.68 & 0.80516 & -0.60208 & -0.70358 & 0.50087 \\
\hline
\end{tabular}

${ }^{\mathrm{D}} \mathrm{IR}$ frequency $(\mathrm{v})$ is reported in $\mathrm{cm}^{-1}$.

Table S11. Measured, predicted, and leave-one-out (LOO) predicted $\Delta \Delta G^{\ddagger}$ of ortho-substituted arylboronic acids in redox-relay Heck reaction (data for Figure 7a).

$\Delta \Delta G_{\text {norm }}^{\ddagger}=1.89+1.01 \sigma_{p}+0.23 L_{o}-0.72 v_{C=0,0}$

\begin{tabular}{cccc}
\hline $\mathbf{R}$ & Measured ${ }^{\mathrm{a}} \boldsymbol{\Delta} \boldsymbol{\Delta} \mathbf{G}^{\ddagger}$ & Predicted ${ }^{\mathrm{a}} \boldsymbol{\Delta} \boldsymbol{\Delta} \mathbf{G}^{\ddagger}$ & POO \\
\hline $2-\mathrm{Me}$ & 2.01 & 1.85 & 1.74 \\
$2-{ }^{\mathrm{C}} \mathrm{P}_{3}$ & 2.47 & 2.50 & 2.59 \\
$2-\mathrm{OMe}$ & 0.89 & 1.15 & 1.32 \\
$2-\mathrm{F}$ & 2.00 & 2.10 & 2.14 \\
$2-\mathrm{Cl}$ & 2.73 & 2.78 & 2.82 \\
$2-i-\mathrm{Pr}$ & 1.88 & 1.84 & 1.82 \\
$2-\mathrm{Ph}$ & 2.27 & 2.16 & 1.85 \\
$\mathrm{H}$ & 0.88 & 0.77 & 0.38 \\
\hline
\end{tabular}

${ }^{\mathrm{a}} \Delta \Delta \mathrm{G}^{\mp}$ is reported in $\mathrm{kcal} / \mathrm{mol}$.

RMS error $\quad 0.183$

$\mathrm{R}^{2} \quad 0.959$

Adjusted $\mathrm{R}^{2} \quad 0.928$

F-stat $\quad 30.9$

Table S12. Measured, predicted, and leave-one-out (LOO) predicted $\Delta \Delta G^{\ddagger}$ of ortho-substituted arylboronic acids in redox-relay Heck reaction (data for Figure 7b).

$\Delta \Delta G^{\ddagger}{ }_{\text {norm }}=1.89+0.97 N B O_{=O, p}+0.27 L_{o}-0.65 v_{C=O, o}$

\begin{tabular}{cccc}
\hline $\mathbf{R}$ & Measured ${ }^{\mathrm{a}} \boldsymbol{\Delta} \boldsymbol{\Delta} \mathbf{G}^{\ddagger}$ & Predicted $^{\mathrm{a}} \boldsymbol{\Delta} \boldsymbol{\Delta} \mathbf{G}^{\ddagger}$ & $\begin{array}{c}\text { LOO } \\
\text { Predicted }\end{array}{ }^{\mathrm{a}} \boldsymbol{\Delta} \boldsymbol{\Delta} \mathbf{G}^{\ddagger}$ \\
\hline 2-Me & 2.01 & 1.96 & 1.91 \\
2-CF & 2.47 & 2.36 & 2.11 \\
2-OMe & 0.89 & 0.72 & 0.32
\end{tabular}




\begin{tabular}{cccc}
$2-\mathrm{F}$ & 2.00 & 2.02 & 2.03 \\
$2-\mathrm{Cl}$ & 2.73 & 2.63 & 2.58 \\
$2-i-\mathrm{Pr}$ & 1.88 & 2.06 & 2.11 \\
$2-\mathrm{Ph}$ & 2.27 & 2.35 & 2.56 \\
$\mathrm{H}$ & 0.88 & 1.05 & 1.32 \\
\hline
\end{tabular}

${ }^{\mathrm{a}} \Delta \Delta \mathrm{G}^{\mp}$ is reported in $\mathrm{kcal} / \mathrm{mol}$.

$\begin{array}{lr}\text { RMS error } & 0.173 \\ \mathrm{R}^{2} & 0.963 \\ \text { Adjusted } \mathrm{R}^{2} & 0.935 \\ \text { F-stat } & 34.7\end{array}$

Table S13. Measured, predicted, and leave-one-out (LOO) predicted $\Delta \Delta G^{\ddagger}$ of ortho-substituted arylboronic acids in redox-relay Heck reaction.

$\Delta \Delta G_{\text {norm }}^{\ddagger}=1.89+1.01 v_{C=0, p}+0.43 L_{o}-0.65 v_{C=0,0}$

\begin{tabular}{cccc}
\hline $\mathbf{R}$ & Measured ${ }^{\mathrm{a}} \boldsymbol{\Delta} \boldsymbol{\Delta} \mathbf{G}^{\ddagger}$ & Predicted $^{\mathrm{a}} \boldsymbol{\Delta} \boldsymbol{\Delta} \mathbf{G}^{\ddagger}$ & $\begin{array}{c}\text { LOO } \\
\text { Predicted }\end{array}{ }^{\mathrm{a}} \boldsymbol{\Delta} \boldsymbol{\Delta} \mathbf{G}^{\ddagger}$ \\
\hline $2-\mathrm{Me}$ & 2.01 & 2.01 & 2.01 \\
$2-\mathrm{CF}_{3}$ & 2.47 & 2.43 & 2.34 \\
$2-\mathrm{OMe}$ & 0.89 & 0.74 & 0.42 \\
$2-\mathrm{F}$ & 2.00 & 2.08 & 2.10 \\
$2-\mathrm{Cl}$ & 2.73 & 2.52 & 2.43 \\
$2-i-\mathrm{Pr}$ & 1.88 & 2.05 & 2.10 \\
$2-\mathrm{Ph}$ & 2.27 & 2.33 & 2.49 \\
$\mathrm{H}$ & 0.88 & 0.98 & 1.16
\end{tabular}

${ }^{\mathrm{a}} \Delta \Delta \mathrm{G}^{\mp}$ is reported in $\mathrm{kcal} / \mathrm{mol}$.

$\begin{array}{lc}\text { RMS error } & 0.17 \\ \mathrm{R}^{2} & 0.97 \\ \text { Adjusted } \mathrm{R}^{2} & 0.94 \\ \text { F-stat } & 36.80\end{array}$

Table S14. Measured, predicted, and leave-one-out (LOO) predicted $\Delta \Delta G^{\ddagger}$ of ortho-, meta- and parasubstituted arylboronic acids in redox-relay Heck reaction (data for Figure 8a).

$\Delta \Delta G^{\ddagger}{ }_{\text {norm }}=1.63+0.97 \sigma_{p / m}+0.27 L_{o}-0.60 v_{C=0,0}$

\begin{tabular}{cccc}
\hline $\mathbf{R}$ & Measured $^{\mathrm{a}} \boldsymbol{\Delta} \boldsymbol{\Delta} \mathbf{G}^{\ddagger}$ & Predicted $^{\mathrm{a}} \boldsymbol{\Delta} \boldsymbol{\Delta} \mathbf{G}^{\ddagger}$ & $\begin{array}{c}\text { LOO } \\
\text { Predicted }\end{array}{ }^{\mathrm{a}} \boldsymbol{\Delta} \boldsymbol{\Delta} \mathbf{G}^{\ddagger}$ \\
\hline $2-\mathrm{CF}_{3}$ & 2.47 & 2.33 & 2.27 \\
$2-\mathrm{Cl}$ & 2.73 & 2.55 & 2.48
\end{tabular}




\begin{tabular}{cccc}
$2-\mathrm{F}$ & 2.00 & 1.95 & 1.93 \\
$2-i-\mathrm{Pr}$ & 1.88 & 1.85 & 1.84 \\
$2-\mathrm{Me}$ & 2.01 & 1.77 & 1.63 \\
$2-\mathrm{OMe}$ & 0.89 & 1.28 & 1.39 \\
$2-\mathrm{Ph}$ & 2.27 & 2.27 & 2.25 \\
$3-\mathrm{CN}$ & 2.31 & 1.91 & 1.81 \\
$3-\mathrm{MeO}$ & 0.97 & 1.21 & 1.24 \\
$3-\mathrm{SO}_{2} \mathrm{Me}$ & 2.23 & 2.25 & 2.26 \\
$4-\mathrm{Cl}$ & 1.36 & 1.43 & 1.44 \\
$4-\mathrm{CO}_{2} \mathrm{Me}$ & 1.74 & 1.94 & 1.97 \\
$4-\mathrm{COMe}_{4-\mathrm{M}}$ & 1.64 & 2.15 & 2.25 \\
$4-\mathrm{MeO}$ & 1.18 & 1.00 & 0.97 \\
$4-t-\mathrm{Bu}$ & 0.44 & 0.62 & 0.67 \\
$\mathrm{H}$ & 0.71 & 0.42 & 0.30 \\
\hline
\end{tabular}

${ }^{\mathrm{a}} \Delta \Delta \mathrm{G}^{\mp}$ is reported in $\mathrm{kcal} / \mathrm{mol}$.

RMS error $\quad 0.268$

$\begin{array}{ll}\mathrm{R}^{2} & 0.877\end{array}$

Adjusted $\mathrm{R}^{2} \quad 0.849$

F-stat $\quad 30.9$

Table S15. Measured, predicted, and leave-one-out (LOO) predicted $\Delta \Delta G^{\ddagger}$ of ortho-, meta- and parasubstituted arylboronic acids in redox-relay Heck reaction (data for Figure 8b).

$\Delta \Delta G_{\text {norm }}^{\ddagger}=1.63+1.00 N B O_{=0, p / m}+0.26 L_{o}-0.62 v_{C=0, o}$

\begin{tabular}{|c|c|c|c|}
\hline $\mathbf{R}$ & Measured ${ }^{\mathrm{a}} \boldsymbol{\Delta} \boldsymbol{\Delta} \mathbf{G}^{\ddagger}$ & Predicted ${ }^{\mathrm{a}} \boldsymbol{\Delta} \boldsymbol{\Delta} \mathbf{G}^{\ddagger}$ & $\begin{array}{c}\text { LOO } \\
\text { Predicted }{ }^{\mathrm{a}} \Delta \Delta \mathrm{G}^{\ddagger}\end{array}$ \\
\hline $2-\mathrm{CF}_{3}$ & 2.47 & 2.30 & 2.23 \\
\hline $2-\mathrm{Cl}$ & 2.73 & 2.57 & 2.51 \\
\hline $2-\mathrm{F}$ & 2.00 & 2.00 & 2.00 \\
\hline $2-i-\mathrm{Pr}$ & 1.88 & 2.06 & 2.10 \\
\hline 2-Me & 2.01 & 1.95 & 1.91 \\
\hline 2-OMe & 0.89 & 0.81 & 0.77 \\
\hline $2-\mathrm{Ph}$ & 2.27 & 2.35 & 2.50 \\
\hline $3-\mathrm{CN}$ & 2.31 & 2.17 & 2.13 \\
\hline 3-MeO & 0.97 & 1.38 & 1.42 \\
\hline $3-\mathrm{SO}_{2} \mathrm{Me}$ & 2.23 & 2.04 & 2.01 \\
\hline 4-Cl & 1.36 & 1.43 & 1.43 \\
\hline $4-\mathrm{CO}_{2} \mathrm{Me}$ & 1.74 & 1.82 & 1.83 \\
\hline 4-COMe & 1.64 & 1.79 & 1.81 \\
\hline $4-F$ & 1.18 & 1.02 & 1.00 \\
\hline
\end{tabular}




\begin{tabular}{|c|c|c|c|}
\hline 4-MeO & 0.44 & 0.16 & 0.00 \\
\hline $4-t-B u$ & 0.71 & 0.80 & 0.82 \\
\hline $\mathrm{H}$ & 0.88 & 1.07 & 1.10 \\
\hline \multicolumn{4}{|c|}{$\Delta \Delta \mathrm{G}^{\mp}$ is reported in $\mathrm{kcal} / \mathrm{mol}$. } \\
\hline RMS error & & & \\
\hline $\mathrm{R}^{2}$ & & & \\
\hline Adjusted $\mathrm{R}^{2}$ & & & \\
\hline F-stat & & & \\
\hline
\end{tabular}

Table S16. Measured, predicted, and leave-one-out (LOO) predicted $\Delta \Delta G^{\ddagger}$ of ortho-, meta- and parasubstituted arylboronic acids in redox-relay Heck reaction.

$\Delta \Delta G^{\ddagger}{ }_{\text {norm }}=1.63+0.97 v_{C=0, p / m}+0.33 L_{o}-0.55 v_{C=0,0}$

\begin{tabular}{cccc}
\hline $\mathbf{R}$ & Measured ${ }^{\mathrm{a}} \boldsymbol{\Delta} \boldsymbol{\Delta} \mathbf{G}^{\ddagger}$ & Predicted ${ }^{\mathrm{a}} \boldsymbol{\Delta} \boldsymbol{\Delta} \mathbf{G}^{\ddagger}$ & $\begin{array}{c}\text { LOO } \\
\text { Predicted }\end{array}{ }^{\mathrm{a}} \boldsymbol{\Delta} \mathbf{G}^{\ddagger}$ \\
\hline $2-\mathrm{CF} 3$ & 2.47 & 2.50 & 2.51 \\
$2-\mathrm{Cl}$ & 2.73 & 2.50 & 2.42 \\
$2-\mathrm{F}$ & 2.00 & 2.11 & 2.15 \\
$2-\mathrm{i}-\mathrm{Pr}$ & 1.88 & 2.03 & 2.07 \\
2-Me & 2.01 & 2.02 & 2.02 \\
2-OMe & 0.89 & 0.87 & 0.86 \\
2-Ph & 2.27 & 2.25 & 2.21 \\
$3-\mathrm{CN}$ & 2.31 & 2.30 & 2.30 \\
3-MeO & 0.97 & 1.17 & 1.19 \\
3-SO ${ }_{2} \mathrm{Me}$ & 2.23 & 1.95 & 1.90 \\
$4-\mathrm{Cl}$ & 1.36 & 1.38 & 1.38 \\
$4-\mathrm{CO}{ }_{2} \mathrm{Me}$ & 1.74 & 1.70 & 1.69 \\
$4-\mathrm{COMe}$ & 1.64 & 1.61 & 1.61 \\
$4-\mathrm{F}$ & 1.18 & 1.20 & 1.20 \\
$4-\mathrm{MeO}$ & 0.44 & 0.12 & -0.08 \\
$4-t-\mathrm{Bu}$ & 0.71 & 0.80 & 0.82 \\
$\mathrm{H}$ & 0.88 & 1.21 & 1.25 \\
\hline
\end{tabular}

${ }^{\mathrm{a}} \Delta \Delta \mathrm{G}^{\mp}$ is reported in $\mathrm{kcal} / \mathrm{mol}$.

$\begin{array}{lr}\text { RMS error } & 0.180 \\ \mathrm{R}^{2} & 0.944 \\ \text { Adjusted } \mathrm{R}^{2} & 0.932 \\ \text { F-stat } & 73.6\end{array}$

\section{B. Linear Regression Models for $\beta$-aryl elimination reaction}


Table S17. Measured, predicted, and leave-one-out (LOO) predicted $\Delta \Delta G^{\ddagger}$ of ortho-, meta- and parasubstituted arylboronic acids in $\beta$-aryl elimination reaction.

FitA: $\Delta \Delta G_{\text {norm }}^{\ddagger}=1.06+0.81 \sigma_{p}+0.46 L_{o}-0.92 v_{C=0,0}$

\begin{tabular}{cccc}
\hline $\mathbf{R}$ & Measured ${ }^{\mathrm{a}} \boldsymbol{\Delta} \boldsymbol{\Delta} \mathbf{G}^{\ddagger}$ & Predicted $^{\mathrm{a}} \boldsymbol{\Delta} \boldsymbol{\Delta} \mathbf{G}^{\ddagger}$ & $\begin{array}{c}\text { LOO } \\
\text { Predicted }\end{array}{ }^{\mathrm{a}} \boldsymbol{\Delta} \boldsymbol{\Delta} \mathbf{G}^{\ddagger}$ \\
\hline 2-Cl & 2.81 & 2.75 & 2.72 \\
2-F & 2.57 & 2.06 & 1.87 \\
2-Me & 1.99 & 2.45 & 2.77 \\
2-OMe & 2.32 & 2.31 & 2.24 \\
3-F & 0.82 & 0.44 & 0.33 \\
3-Me & -0.06 & 0.29 & 0.35 \\
3-MeO & 0.00 & 0.56 & 0.64 \\
4-CF 3 & 0.65 & 0.68 & 0.69 \\
4-Cl & 0.57 & 0.62 & 0.63 \\
4-F & 0.74 & 0.39 & 0.34 \\
4-Me & 0.11 & 0.23 & 0.25 \\
4-NMe & 0.63 & 0.37 & -0.03 \\
4-OMe & 0.65 & 0.64 & 0.64 \\
\hline
\end{tabular}

${ }^{\mathrm{a}} \Delta \Delta \mathrm{G}^{\mp}$ is reported in $\mathrm{kcal} / \mathrm{mol}$.

$\begin{array}{lc}\text { RMS error } & 0.38 \\ \mathrm{R}^{2} & 0.90 \\ \text { Adjusted } \mathrm{R}^{2} & 0.86 \\ \text { F-stat } & 14.12\end{array}$

Table S18. Measured, predicted, and leave-one-out (LOO) predicted $\Delta \Delta G^{\ddagger}$ of ortho-, meta- and parasubstituted arylboronic acids in $\beta$-aryl elimination reaction (data for Figure 9a).

FitB: $\Delta \Delta G_{\text {norm }}^{\ddagger}=1.06+0.66 N B O_{=0, p}+0.58 L_{o}-0.75 v_{C=0, o}$

\begin{tabular}{cccc}
\hline $\mathbf{R}$ & Measured ${ }^{\mathrm{a}} \boldsymbol{\Delta} \boldsymbol{\Delta} \mathbf{G}^{\ddagger}$ & Predicted ${ }^{\mathrm{a}} \boldsymbol{\Delta} \boldsymbol{\Delta} \mathbf{G}^{\ddagger}$ & $\begin{array}{c}\text { LOO } \\
\text { Predicted }\end{array}{ }^{\mathrm{a}} \boldsymbol{\Delta} \boldsymbol{\Delta} \mathbf{G}^{\ddagger}$ \\
\hline 2-Cl & 2.81 & 2.69 & 2.61 \\
2-F & 2.57 & 1.96 & 1.75 \\
2-Me & 1.99 & 2.45 & 2.85 \\
2-OMe & 2.32 & 2.39 & 3.67 \\
3-F & 0.82 & 0.52 & 0.43 \\
3-Me & -0.06 & 0.54 & 0.62 \\
3-MeO & 0.00 & 0.60 & 0.68 \\
$4-\mathrm{CF} 3$ & 0.65 & 0.54 & 0.49 \\
4-Cl & 0.57 & 0.54 & 0.53 \\
$4-\mathrm{F}$ & 0.74 & 0.39 & 0.34 \\
$4-\mathrm{Me}$ & 0.11 & 0.36 & 0.40
\end{tabular}




$\begin{array}{llll}4-\mathrm{NMe}_{2} & 0.63 & 0.45 & 0.20 \\ 4-\mathrm{OMe} & 0.65 & 0.37 & 0.29 \\ { }^{\mathrm{a}} \Delta \Delta \mathrm{G}^{\mp} \text { is reported in kcal/mol. } & & \\ & & & \\ \mathrm{RMS} \text { error } & 0.44 & & \\ \mathrm{R}^{2} & 0.86 & & \\ \text { Adjusted } \mathrm{R}^{2} & 0.81 & & \\ \text { F-stat } & 17.90 & \end{array}$

Table S19. Measured, predicted, and leave-one-out (LOO) predicted $\Delta \Delta G^{\ddagger}$ of ortho-, meta- and parasubstituted arylboronic acids in $\beta$-aryl elimination reaction.

FitC: $\Delta \Delta G_{\text {norm }}^{\ddagger}=1.06+0.67 v_{C=0, p}+0.56 L_{o}-0.75 v_{C=0,0}$

\begin{tabular}{|c|c|c|c|}
\hline $\mathbf{R}$ & Measured ${ }^{\mathrm{a}} \boldsymbol{\Delta} \boldsymbol{\Delta} \mathbf{G}^{\ddagger}$ & Predicted ${ }^{\mathrm{a}} \boldsymbol{\Delta} \boldsymbol{\Delta} \mathbf{G}^{\ddagger}$ & $\begin{array}{c}\text { LOO } \\
\text { Predicted }{ }^{\mathrm{a}} \boldsymbol{\Delta} \boldsymbol{\Delta} \mathrm{G}^{\ddagger}\end{array}$ \\
\hline $2-\mathrm{Cl}$ & 2.81 & 2.61 & 2.52 \\
\hline $2-\mathrm{F}$ & 2.57 & 2.04 & 1.83 \\
\hline 2-Me & 1.99 & 2.54 & 3.09 \\
\hline 2-OMe & 2.32 & 2.37 & 3.08 \\
\hline $3-F$ & 0.82 & 0.53 & 0.44 \\
\hline 3-Me & -0.06 & 0.47 & 0.54 \\
\hline 3-MeO & 0.00 & 0.48 & 0.55 \\
\hline $4-\mathrm{CF}_{3}$ & 0.65 & 0.54 & 0.50 \\
\hline $4-\mathrm{Cl}$ & 0.57 & 0.50 & 0.49 \\
\hline $4-F$ & 0.74 & 0.49 & 0.45 \\
\hline 4-Me & 0.11 & 0.46 & 0.51 \\
\hline $4-\mathrm{NMe}_{2}$ & 0.63 & 0.35 & -0.14 \\
\hline 4-OMe & 0.65 & 0.42 & 0.36 \\
\hline \multicolumn{4}{|c|}{${ }^{a} \Delta \Delta \mathrm{G}^{\mp}$ is reported in $\mathrm{kcal} / \mathrm{mol}$. } \\
\hline RMS error & 0.42 & & \\
\hline $\mathrm{R}^{2}$ & 0.87 & & \\
\hline Adjusted $\mathrm{R}^{2}$ & 0.83 & & \\
\hline F-stat & 20.10 & & \\
\hline
\end{tabular}

Table S20. Measured, predicted, and leave-one-out (LOO) predicted $\Delta \Delta G^{\ddagger}$ of ortho-, meta- and parasubstituted arylboronic acids in $\beta$-aryl elimination reaction (data for Figure 9b).

FitForward: $\Delta \Delta G_{\text {norm }}^{\ddagger}=1.06+0.85 B_{1}+0.30 v_{\mathrm{COH}}$ 


\begin{tabular}{cccc}
$2-\mathrm{Cl}$ & 2.81 & 3.02 & 3.17 \\
$2-\mathrm{F}$ & 2.57 & 2.52 & 2.46 \\
$2-\mathrm{Me}$ & 1.99 & 1.98 & 1.93 \\
$2-\mathrm{OMe}$ & 2.32 & 2.07 & 2.02 \\
$3-\mathrm{F}$ & 0.82 & 0.55 & 0.52 \\
$3-\mathrm{Me}$ & -0.06 & 0.38 & 0.44 \\
$3-\mathrm{MeO}$ & 0.00 & 0.46 & 0.52 \\
$4-\mathrm{CF}_{3}$ & 0.65 & 0.78 & 0.81 \\
$4-\mathrm{Cl}$ & 0.57 & 0.37 & 0.34 \\
$4-\mathrm{F}$ & 0.74 & 0.43 & 0.39 \\
$4-\mathrm{Me}$ & 0.11 & 0.34 & 0.38 \\
$4-\mathrm{NMe}$ & 0.63 & 0.44 & 0.42 \\
$4-\mathrm{OMe}$ & 0.65 & 0.46 & 0.44 \\
\hline
\end{tabular}

${ }^{\mathrm{a}} \Delta \Delta \mathrm{G}^{\mp}$ is reported in $\mathrm{kcal} / \mathrm{mol}$.

$\begin{array}{lc}\text { RMS error } & 0.29 \\ \mathrm{R}^{2} & 0.93 \\ \text { Adjusted } \mathrm{R}^{2} & 0.91 \\ \text { F-stat } & 64.20\end{array}$

\section{Linear Regression Models for Enzyme-catalyzed Benzoin Condensation}

Table S21. Measured, predicted, and leave-one-out (LOO) predicted $\Delta \Delta G^{\ddagger}$ of meta-substituted arylboronic acids in enzymatic benzoin condensation reaction (data for Figure 10a).

\begin{tabular}{ccc}
\hline $\mathbf{R}$ & Measured $^{\mathrm{a}} \boldsymbol{\Delta} \boldsymbol{\Delta} \mathbf{G}^{\ddagger}$ & $\boldsymbol{\sigma}_{\mathrm{m}}$ \\
\hline 3-H & 1.88 & 0 \\
$3-\mathrm{F}$ & 1.58 & 0.34 \\
3-Cl & 2.06 & 0.37 \\
3-Br & 2.31 & 0.39 \\
3-Me & 2.48 & -0.07 \\
3-OMe & 2.31 & 0.12 \\
3-OEt & 2.48 & 0.1 \\
3-Oi-Pr & 3.14 & 0.1 \\
3-OPh & 3.14 & 0.25 \\
3-OAc & 3.14 & 0.39 \\
3-OH & 1.88 & 0.12 \\
3-CN & 1.88 & 0.56 \\
\hline
\end{tabular}

${ }^{\mathrm{a}} \Delta \Delta \mathrm{G}^{\ddagger}$ is reported in $\mathrm{kcal} / \mathrm{mol}$. 
Table S22. Measured, predicted, and leave-one-out (LOO) predicted $\Delta \Delta G^{\ddagger}$ of meta-substituted arylboronic acids in enzymatic benzoin condensation reaction.

FitA: $\Delta \Delta G_{\text {norm }}^{\ddagger}=2.36-0.27 \sigma_{p / m}+0.46 L_{m}-0.92 v_{C=0, m}$

\begin{tabular}{cccc}
\hline $\mathbf{R}$ & Measured ${ }^{\mathrm{a}} \boldsymbol{\Delta} \boldsymbol{\Delta} \mathbf{G}^{\ddagger}$ & Predicted ${ }^{\mathrm{a}} \boldsymbol{\Delta} \boldsymbol{\Delta} \mathbf{G}^{\ddagger}$ & $\begin{array}{c}\text { LOO } \\
\text { Predicted }\end{array}{ }^{\mathrm{a}} \boldsymbol{\Delta} \boldsymbol{\Delta} \mathbf{G}^{\ddagger}$ \\
\hline 3-H & 1.88 & 1.79 & 1.74 \\
3-F & 1.58 & 1.79 & 1.85 \\
3-Cl & 2.06 & 2.03 & 2.03 \\
3-Br & 2.31 & 2.08 & 1.78 \\
3-Me & 2.48 & 2.25 & 2.13 \\
3-OMe & 2.31 & 2.53 & 2.58 \\
3-OEt & 2.48 & 2.98 & 3.19 \\
3-Oi-Pr & 3.14 & 2.99 & 2.93 \\
3-OPh & 3.14 & 3.04 & 2.97 \\
3-OAC & 3.14 & 2.59 & 2.21 \\
3-OH & 1.88 & 2.00 & 2.03 \\
3-CN & 1.88 & 2.18 & 2.45 \\
${ }^{\mathrm{a}} \Delta \Delta \mathbf{G}^{\ddagger}$ is reported in kcal/mol. & &
\end{tabular}

RMS error $\quad 0.333$

$\mathrm{R}^{2} \quad 0.725$

Adjusted $\mathrm{R}^{2} \quad 0.621$

F-stat $\quad 7.02$

Table S23. Measured, predicted, and leave-one-out (LOO) predicted $\Delta \Delta G^{\ddagger}$ of meta-substituted arylboronic acids in enzymatic benzoin condensation reaction.

FitC: $\Delta \Delta G_{\text {norm }}^{\ddagger}=2.36-0.41 v_{C=0, p}+0.17 v_{C=0, m}+0.48 I_{C=0, m}$

\begin{tabular}{cccc}
\hline $\mathbf{R}$ & Measured ${ }^{\mathrm{a}} \boldsymbol{\Delta} \boldsymbol{\Delta} \mathbf{G}^{\ddagger}$ & Predicted $^{\mathrm{a}} \boldsymbol{\Delta} \boldsymbol{\Delta} \mathbf{G}^{\ddagger}$ & $\begin{array}{c}\text { LOO } \\
\text { Predicted }\end{array}{ }^{\mathrm{a}} \boldsymbol{\Delta} \boldsymbol{\Delta} \mathbf{G}^{\ddagger}$ \\
\hline 3-H & 1.88 & 1.95 & 1.99 \\
3-F & 1.58 & 1.49 & 1.44 \\
3-Cl & 2.06 & 2.05 & 2.05 \\
3-Br & 2.31 & 2.18 & 2.16 \\
3-Me & 2.48 & 2.43 & 2.39 \\
3-OMe & 2.31 & 2.38 & 2.39 \\
3-OEt & 2.48 & 2.68 & 2.73 \\
3-Oi-Pr & 3.14 & 2.96 & 2.88 \\
3-OPh & 3.14 & 3.16 & 3.18 \\
3-OAC & 3.14 & 3.12 & 3.09 \\
3-OH & 1.88 & 1.88 & 1.87
\end{tabular}




$\begin{array}{ll}\text { 3-CN } & 1.88 \\ { }^{\mathrm{a}} \Delta \Delta \mathrm{G}^{\mp} \text { is reported in kcal/mol. } \\ & \\ & \\ \text { RMS error } & 0.122 \\ \mathrm{R}^{2} & 0.963 \\ \text { Adjusted } \mathrm{R}^{2} & 0.949 \\ \text { F-stat } & 69.1\end{array}$

Table S24. Measured, predicted, and leave-one-out (LOO) predicted $\Delta \Delta G^{\ddagger}$ of meta-substituted arylboronic acids in enzymatic benzoin condensation reaction (data for Figure 10b).

FitD: $\Delta \Delta G^{\ddagger}{ }_{n o r m}=2.36-0.46 N B O_{=0, p}+0.22 v_{C=O, m}+0.51 I_{C=0, m}$

\begin{tabular}{cccc}
\hline $\mathbf{R}$ & Measured ${ }^{\mathrm{a}} \boldsymbol{\Delta} \boldsymbol{\Delta} \mathbf{G}^{\ddagger}$ & Predicted ${ }^{\mathrm{a}} \boldsymbol{\Delta} \boldsymbol{\Delta} \mathbf{G}^{\ddagger}$ & $\begin{array}{c}\text { LOO } \\
\text { Predicted }\end{array}{ }^{\mathrm{a}} \boldsymbol{\Delta} \boldsymbol{\Delta} \mathbf{G}^{\ddagger}$ \\
\hline 3-H & 1.88 & 1.95 & 2.01 \\
3-F & 1.58 & 1.54 & 1.52 \\
3-Cl & 2.06 & 2.06 & 2.06 \\
3-Br & 2.31 & 2.17 & 2.14 \\
3-Me & 2.48 & 2.45 & 2.43 \\
3-OMe & 2.31 & 2.34 & 2.35 \\
3-OEt & 2.48 & 2.72 & 2.79 \\
3-Oi-Pr & 3.14 & 2.91 & 2.83 \\
3-OPh & 3.14 & 3.17 & 3.19 \\
3-OAC & 3.14 & 3.14 & 3.14 \\
3-OH & 1.88 & 1.85 & 1.83 \\
3-CN & 1.88 & 1.95 & 2.02 \\
\hline${ }^{\mathrm{a}} \Delta \mathrm{G}^{\mp}$ is reported in kcal/mol. & &
\end{tabular}

$\begin{array}{lc}\text { RMS error } & 0.133 \\ \mathrm{R}^{2} & 0.956 \\ \text { Adjusted } \mathrm{R}^{2} & 0.94 \\ \text { F-stat } & 58.2\end{array}$




\section{Cartesian Coordinates}

\section{A. Cartesian Coordinates of geometry optimized para-substituted benzoic acids}

$\begin{array}{lrrr}\text { 4-H } & & & \\ \text { C } & -0.44402200 & -1.19534300 & 0.00000300 \\ \text { C } & 0.21414500 & 0.03015800 & 0.00000100 \\ \text { C } & -0.51131500 & 1.21709800 & -0.00000900 \\ \text { C } & -1.89521100 & 1.17807200 & -0.00001800 \\ \text { C } & -2.55361800 & -0.04512600 & -0.00001800 \\ \text { C } & -1.83002100 & -1.22936000 & -0.00000600 \\ \text { C } & 1.69655400 & 0.12263600 & 0.00001000 \\ \text { O } & 2.31942600 & 1.15033800 & 0.00001000 \\ H & 0.02644800 & 2.15487200 & -0.00000900 \\ \text { O } & 2.30214000 & -1.08088000 & 0.00002000 \\ H & 3.25377400 & -0.91298100 & 0.00002600 \\ H & 0.12950800 & -2.11060000 & 0.00001300 \\ H & -2.34520100 & -2.17929700 & -0.00000400 \\ H & -2.46171800 & 2.09828100 & -0.00002400 \\ H & -3.63440400 & -0.07475400 & -0.00002000\end{array}$

\section{4-NMe ${ }_{2}$}

C

$0.77795100 \quad 1.17052800$

0.00024700

C

$1.47150200-0.03699700$

0.00010400

$0.74550300-1.22565200$

0.00010900

$-0.63162000-1.21869600$

0.00032200

$-1.34452300-0.00118900$

0.00064200

$-0.60098200 \quad 1.19649200$

0.00045200

$2.94139400-0.10730700$

$-0.00016500$

$\begin{array}{lll}3.59010600 & -1.12275100 & -0.00034700\end{array}$

$1.28704600-2.16183100-0.00012300$

$\mathrm{H}$

$\begin{array}{lll}3.53463100 & 1.10794200 & -0.00019500\end{array}$

$\mathrm{H}$

$4.48653400 \quad 0.94456800$

$-0.00038000$

$1.32890000 \quad 2.10021100$

0.00014300

$-1.10455600 \quad 2.15034700$

0.00047600

$-1.15948800-2.15935300$

0.00019100

$-2.71133200 \quad 0.01605500$

0.00110600

$-3.41786200 \quad 1.27780800$

$-0.00080200$

$-4.48660400 \quad 1.08734100$

$-0.00044300$

$\begin{array}{lll}-3.17756800 & 1.87386100 & 0.88366800\end{array}$

$\begin{array}{lll}-3.17757000 & 1.87144500 & -0.88694500\end{array}$

$-3.44996500-1.22743100-0.00049600$

$\begin{array}{lll}-4.51343600 & -1.00941000 & 0.00050900\end{array}$

$\begin{array}{lll}-3.22559500 & -1.82697200 & -0.88675800\end{array}$

$\mathrm{H}$

$-3.22462800-1.82944700$

0.88377800

\section{4-NEt ${ }_{2}$}

C

$\begin{array}{lll}1.36438300 & 1.12640700 & 0.30069100\end{array}$

C

$\begin{array}{lll}2.06180900 & -0.03834400 & -0.00911100\end{array}$ 


$\begin{array}{lrrr}\mathrm{C} & 1.33481000 & -1.18621500 & -0.31526400 \\ \mathrm{C} & -0.04184400 & -1.18080300 & -0.31264000 \\ \mathrm{C} & -0.76245600 & -0.00646000 & -0.00254700 \\ \mathrm{C} & -0.01411100 & 1.15001900 & 0.30383900 \\ \mathrm{C} & 3.53052300 & -0.10476300 & -0.02561900 \\ \mathrm{O} & 4.18146500 & -1.08480600 & -0.28727500 \\ \mathrm{H} & 1.87555800 & -2.09304900 & -0.55006500 \\ \mathrm{O} & 4.12265200 & 1.07008300 & 0.28926500 \\ \mathrm{H} & 5.07456900 & 0.91257600 & 0.24715700 \\ \mathrm{H} & 1.91232200 & 2.02803900 & 0.53494200 \\ \mathrm{H} & -0.51324800 & 2.07954000 & 0.52835500 \\ \mathrm{H} & -0.56295500 & -2.09872500 & -0.53497500 \\ \mathrm{~N} & -2.13059100 & 0.00887500 & 0.00062600 \\ \mathrm{C} & -2.88042700 & 1.17080000 & 0.44383300 \\ \mathrm{H} & -3.83642700 & 0.81329300 & 0.82970700 \\ \mathrm{H} & -2.36906500 & 1.63273800 & 1.28802000 \\ \mathrm{C} & -2.90892800 & -1.13604700 & -0.43825900 \\ \mathrm{H} & -3.85804600 & -0.75679600 & -0.82032200 \\ \mathrm{H} & -2.41210800 & -1.61037200 & -1.28417000 \\ \mathrm{C} & -3.16528000 & -2.14423200 & 0.67653300 \\ \mathrm{H} & -3.75663800 & -2.98366000 & 0.31118700 \\ \mathrm{H} & -2.22636900 & -2.52728900 & 1.07441400 \\ \mathrm{H} & -3.70892200 & -1.67344800 & 1.49591500 \\ \mathrm{C} & -3.11842900 & 2.18596000 & -0.66877000 \\ \mathrm{H} & -3.68773600 & 3.03886800 & -0.29959000 \\ \mathrm{H} & -2.17266000 & 2.54679000 & -1.07123400 \\ \mathrm{H} & -3.67736800 & 1.72922200 & -1.48576000\end{array}$

$\begin{array}{lrrr}\text { 4-NH } & & & \\ \mathrm{C} & & & \\ \mathrm{C} & 0.00223700 & -1.18005200 & -0.00320000 \\ \mathrm{C} & -0.68016000 & 0.03432300 & -0.00179900 \\ \mathrm{C} & 0.04750200 & 1.22249600 & -0.00378900 \\ \mathrm{C} & 1.42531000 & 1.20277700 & -0.00642800 \\ \mathrm{C} & 2.11441600 & -0.01604700 & -0.00614700 \\ \mathrm{C} & 1.38193900 & -1.20787500 & -0.00585100 \\ \mathrm{C} & -2.15222300 & 0.11283800 & 0.00307400 \\ \mathrm{O} & -2.79272100 & 1.13256700 & 0.00633600 \\ \mathrm{H} & -0.49193600 & 2.15966500 & -0.00205800 \\ \mathrm{O} & -2.75067000 & -1.09850400 & 0.00389800 \\ \mathrm{H} & -3.70223100 & -0.93245500 & 0.00659500 \\ \mathrm{H} & -0.55619200 & -2.10500100 & -0.00131000 \\ \mathrm{H} & 1.90693000 & -2.15429800 & -0.01112000 \\ \mathrm{H} & 1.98445800 & 2.12942700 & -0.01205000 \\ \mathrm{~N} & 3.49375300 & -0.04048100 & -0.05600500 \\ \mathrm{H} & 3.97248200 & 0.79400600 & 0.23772300 \\ \mathrm{H} & 3.94322100 & -0.89123500 & 0.23722100\end{array}$

4-0-iPr

C

$\begin{array}{lll}-1.38193600 & 1.27486500 & -0.19030700\end{array}$ 


$\begin{array}{lrrr}\text { C } & -1.90302400 & -0.00003200 & 0.03431500 \\ \text { C } & -1.03492800 & -1.05835600 & 0.26524600 \\ \text { C } & 0.33636300 & -0.86712800 & 0.27475500 \\ \text { C } & 0.85220300 & 0.41087500 & 0.05214900 \\ \text { C } & -0.01968400 & 1.47807300 & -0.18216500 \\ \text { C } & -3.35492600 & -0.27160800 & 0.03472700 \\ \text { O } & -3.85759100 & -1.34939400 & 0.21956500 \\ H & -1.45136900 & -2.04151100 & 0.43653900 \\ \text { O } & -4.10053500 & 0.82923000 & -0.19771700 \\ H & -5.02364700 & 0.54517200 & -0.17626300 \\ H & -2.05247100 & 2.10247500 & -0.37065000 \\ H & 0.40513800 & 2.45719000 & -0.35147700 \\ H & 0.98560100 & -1.71018000 & 0.45133100 \\ \text { O } & 2.16442400 & 0.72531000 & 0.05845500 \\ \text { C } & 3.15012100 & -0.29900300 & 0.22014400 \\ \text { C } & 4.42399300 & 0.41366700 & 0.62445800 \\ \text { C } & 3.31833800 & -1.08016600 & -1.07210500 \\ H & 2.83737200 & -0.96022100 & 1.03226300 \\ H & 4.27678100 & 0.96833200 & 1.54913600 \\ H & 5.22642800 & -0.30840200 & 0.77032500 \\ H & 4.72137300 & 1.11242200 & -0.15734600 \\ H & 2.38144800 & -1.52715700 & -1.39998200 \\ H & 3.67239700 & -0.41136600 & -1.85683200 \\ H & 4.05144800 & -1.87504500 & -0.93675500\end{array}$

$\begin{array}{lrrr}\text { 4-OBn } & & & \\ \text { C } & -2.58170400 & -1.06260800 & 0.63453500 \\ \text { C } & -2.70183300 & 0.13952300 & -0.06319900 \\ \text { C } & -1.61611600 & 0.62524400 & -0.77938700 \\ \text { C } & -0.41731600 & -0.06708600 & -0.81221300 \\ \text { C } & -0.30886400 & -1.27129700 & -0.11832100 \\ \text { C } & -1.39500600 & -1.76265500 & 0.60779100 \\ \text { C } & -3.95254800 & 0.92711000 & -0.06735500 \\ \text { O } & -4.11327600 & 1.96690300 & -0.65098700 \\ \text { H } & -1.72181200 & 1.56196300 & -1.30895500 \\ \text { O } & -4.93650500 & 0.36302500 & 0.66410300 \\ \text { H } & -5.70086000 & 0.94993100 & 0.59580200 \\ \text { H } & -3.42389200 & -1.44321100 & 1.19377800 \\ \text { H } & -1.27954500 & -2.69857100 & 1.13570000 \\ \text { H } & 0.42032600 & 0.34204500 & -1.35585100 \\ \text { O } & 0.80633900 & -2.04021700 & -0.09024600 \\ \text { C } & 1.93208200 & -1.63616700 & -0.84578800 \\ \text { H } & 1.63917100 & -1.43093900 & -1.87927700 \\ \text { C } & 2.66496100 & -0.45245000 & -0.26144600 \\ \text { C } & 2.54618800 & -0.12562300 & 1.08322200 \\ \text { C } & 3.49632200 & 0.30705200 & -1.07711400 \\ \text { C } & 3.25438100 & 0.94778300 & 1.60538400 \\ \text { H } & 1.89140100 & -0.70840000 & 1.71713500 \\ \text { C } & 4.20946600 & 1.37566400 & -0.55459200\end{array}$




$\begin{array}{lrrr}H & 3.58316100 & 0.06312500 & -2.12956800 \\ \mathrm{C} & 4.08888500 & 1.69884700 & 0.79004400 \\ \mathrm{H} & 3.15158700 & 1.19919200 & 2.65216600 \\ \mathrm{H} & 4.85228200 & 1.96060200 & -1.19811600 \\ \mathrm{H} & 4.63828200 & 2.53574600 & 1.19845100 \\ \mathrm{H} & 2.58404700 & -2.50919500 & -0.85358900\end{array}$

$\begin{array}{lrrr}\text { 4-OH } & & & \\ \text { C } & -0.00980300 & -1.18782900 & 0.00001200 \\ \text { C } & 0.66270600 & 0.03268600 & 0.00000600 \\ \text { C } & -0.06454400 & 1.21830700 & -0.00001300 \\ \text { C } & -1.44561400 & 1.19048700 & -0.00002500 \\ \text { C } & -2.10913500 & -0.03388000 & -0.00001800 \\ \text { C } & -1.39024900 & -1.22520100 & 0.00000000 \\ \text { C } & 2.13841200 & 0.12004200 & 0.00001800 \\ \text { O } & 2.76862300 & 1.14491400 & 0.00001500 \\ \text { H } & 0.47151200 & 2.15716400 & -0.00001800 \\ \text { O } & 2.74112500 & -1.08671700 & 0.00003300 \\ \text { H } & 3.69276900 & -0.91988400 & 0.00004000 \\ \text { H } & 0.55606200 & -2.10799600 & 0.00002600 \\ \text { H } & -1.93078300 & -2.16068400 & 0.00000400 \\ \text { H } & -2.01422800 & 2.11237300 & -0.00004100 \\ \text { O } & -3.46146900 & -0.12361600 & -0.00002700 \\ \text { H } & -3.85220600 & 0.75471500 & -0.00005400\end{array}$

$\begin{array}{lrrr}\text { 4-OMe } & & & \\ \mathrm{C} & -0.54036400 & -1.25921700 & -0.00000100 \\ \mathrm{C} & -1.10322000 & 0.01844700 & 0.00001000 \\ \mathrm{C} & -0.27295300 & 1.13031900 & 0.00003700 \\ \mathrm{C} & 1.10518200 & 0.98855300 & 0.00005900 \\ \mathrm{C} & 1.65889000 & -0.29081900 & 0.00006200 \\ \mathrm{C} & 0.82810500 & -1.41383700 & 0.00002300 \\ \mathrm{C} & -2.56500500 & 0.23625900 & -0.00001300 \\ \mathrm{O} & -3.10388200 & 1.31210600 & -0.00000200 \\ \mathrm{H} & -0.72332700 & 2.11335600 & 0.00003000 \\ \mathrm{O} & -3.27191500 & -0.91314400 & -0.00005100 \\ \mathrm{H} & -4.20495200 & -0.66250400 & -0.00006400 \\ \mathrm{H} & -1.18431500 & -2.12656000 & -0.00002100 \\ \mathrm{H} & 1.28665600 & -2.39234900 & 0.00002000 \\ \mathrm{H} & 1.73158200 & 1.86698300 & 0.00006100 \\ \mathrm{O} & 2.98595500 & -0.54028400 & 0.00005700 \\ \mathrm{C} & 3.86948900 & 0.56222200 & -0.00011000 \\ \mathrm{H} & 4.87193800 & 0.14617600 & -0.00032700 \\ \mathrm{H} & 3.73002700 & 1.17697800 & -0.89167400 \\ \mathrm{H} & 3.73038400 & 1.17693400 & 0.89154100\end{array}$

4-OSiMe

$\begin{array}{llll}\text { C } & -2.02798000 & -1.33929600 & 0.00011600 \\ \text { C } & -2.49122900 & -0.02458400 & 0.00005400\end{array}$




$\begin{array}{lrrr}\mathrm{C} & -1.57694400 & 1.02258800 & 0.00010500 \\ \mathrm{C} & -0.21779500 & 0.77042600 & 0.00020600 \\ \mathrm{C} & 0.24455700 & -0.54719400 & 0.00025900 \\ \mathrm{C} & -0.67283800 & -1.59923000 & 0.00022500 \\ \mathrm{C} & -3.93157800 & 0.30492600 & -0.00006200 \\ \mathrm{O} & -4.38600700 & 1.41928700 & -0.00010600 \\ \mathrm{H} & -1.95054700 & 2.03727100 & 0.00006700 \\ \mathrm{O} & -4.72604700 & -0.78605900 & -0.00011900 \\ \mathrm{H} & -5.63642500 & -0.46276600 & -0.00019100 \\ \mathrm{H} & -2.73645400 & -2.15490700 & 0.00008400 \\ \mathrm{H} & -0.29328200 & -2.61098100 & 0.00028600 \\ \mathrm{H} & 0.48311100 & 1.59283800 & 0.00026500 \\ \mathrm{O} & 1.55173100 & -0.86663100 & 0.00041900 \\ \mathrm{C} & 4.32071700 & -1.14956100 & 0.00014600 \\ \mathrm{C} & 2.98297400 & 1.13920400 & 1.54599200 \\ \mathrm{H} & 4.26710700 & -1.78749400 & -0.88218300 \\ \mathrm{H} & 5.28986600 & -0.64922500 & -0.00028100 \\ \mathrm{H} & 4.26753000 & -1.78680300 & 0.88299800 \\ \mathrm{H} & 2.19700100 & 1.89294800 & 1.57489000 \\ \mathrm{H} & 2.88153100 & 0.51242100 & 2.43270000 \\ \mathrm{H} & 3.94216000 & 1.65614200 & 1.60989700 \\ \mathrm{Si} & 2.93418500 & 0.09130800 & -0.00004900 \\ \mathrm{C} & 2.98241900 & 1.13807300 & -1.54684700 \\ \mathrm{H} & 2.88079300 & 0.51058800 & -2.43304000 \\ \mathrm{H} & 2.19628500 & 1.89163800 & -1.57606700 \\ \mathrm{H} & 3.94149200 & 1.65513200 & -1.61145000\end{array}$

$\begin{array}{lrrr}\text { 4-OEt } & & & \\ \text { C } & 1.03386400 & 1.25646000 & -0.00000800 \\ \text { C } & 1.60138700 & -0.01905700 & 0.00004600 \\ \text { C } & 0.77460800 & -1.13369600 & 0.00014300 \\ \text { C } & -0.60388400 & -0.99704100 & 0.00016300 \\ \text { C } & -1.16331100 & 0.28054800 & 0.00009900 \\ \text { C } & -0.33522200 & 1.40597000 & -0.00003000 \\ \text { C } & 3.06356400 & -0.23164100 & -0.00001100 \\ \text { O } & 3.60678700 & -1.30542900 & -0.00014600 \\ \text { H } & 1.22834600 & -2.11520900 & 0.00020800 \\ \text { O } & 3.76662900 & 0.92037300 & -0.00000200 \\ \text { H } & 4.70037300 & 0.67243400 & -0.00028200 \\ \text { H } & 1.67462500 & 2.12619200 & -0.00010200 \\ \text { H } & -0.79684400 & 2.38306700 & -0.00012800 \\ \text { H } & -1.22666800 & -1.87789900 & 0.00031100 \\ \text { O } & -2.49022100 & 0.52565400 & 0.00022300 \\ \text { C } & -3.38418400 & -0.57832800 & -0.00026400 \\ \text { H } & -3.20336000 & -1.19267800 & -0.88667900 \\ \text { H } & -3.20346600 & -1.19354000 & 0.88560000 \\ \text { C } & -4.78943900 & -0.02626300 & -0.00005700 \\ \text { H } & -5.51029400 & -0.84255900 & -0.00042700 \\ \text { H } & -4.95630300 & 0.58646200 & 0.88407900\end{array}$




$\begin{array}{lrrr}\text { 4-Cyclopropyl } & & \\ \mathrm{C} & -0.89131700 & -1.26888400 & -0.00005900 \\ \mathrm{C} & -1.43212300 & 0.01170300 & -0.00003600 \\ \mathrm{C} & -0.58508600 & 1.11667300 & -0.00012500 \\ \mathrm{C} & 0.78465800 & 0.94164900 & -0.00020500 \\ \mathrm{C} & 1.34275000 & -0.33950900 & -0.00013300 \\ \mathrm{C} & 0.48312700 & -1.43683400 & -0.00009400 \\ \mathrm{C} & -2.89372600 & 0.25019300 & 0.00004800 \\ \mathrm{O} & -3.41487800 & 1.33410700 & 0.00007000 \\ \mathrm{H} & -1.02213500 & 2.10562500 & -0.00013700 \\ \mathrm{O} & -3.61718900 & -0.88804200 & 0.00010600 \\ \mathrm{H} & -4.54660200 & -0.62408400 & 0.00016100 \\ \mathrm{H} & -1.54713200 & -2.12739600 & -0.00004900 \\ \mathrm{H} & 0.90078700 & -2.43530600 & -0.00014100 \\ \mathrm{H} & 1.42770600 & 1.81262700 & -0.00033900 \\ \mathrm{C} & 2.80788500 & -0.55547400 & 0.00011800 \\ \mathrm{C} & 3.71819700 & 0.38630000 & -0.74500400 \\ \mathrm{C} & 3.71801100 & 0.38646400 & 0.74522200 \\ \mathrm{H} & 3.10261800 & -1.59531400 & 0.00028700 \\ \mathrm{H} & 3.24780600 & 1.21332100 & -1.25676600 \\ \mathrm{H} & 4.56601700 & -0.04278900 & -1.25700000 \\ \mathrm{H} & 4.56570000 & -0.04252500 & 1.25751400 \\ \mathrm{H} & 3.24751900 & 1.21362700 & 1.25666900\end{array}$

$\begin{array}{lrrr}\text { 4-t-Bu } & & & \\ \mathrm{C} & -1.06214200 & -1.15998000 & 0.00004700 \\ \mathrm{C} & -1.75899200 & 0.04532100 & -0.00000700 \\ \mathrm{C} & -1.05527600 & 1.24083700 & -0.00005800 \\ \mathrm{C} & 0.33109500 & 1.23503000 & -0.00005600 \\ \mathrm{C} & 1.04715400 & 0.03925100 & -0.00000200 \\ \mathrm{C} & 0.31989700 & -1.15480600 & 0.00004800 \\ \mathrm{C} & -3.24028800 & 0.10091800 & -0.00001200 \\ \mathrm{O} & -3.89141700 & 1.11161800 & -0.00005500 \\ \mathrm{H} & -1.60769600 & 2.17036500 & -0.00010100 \\ \mathrm{O} & -3.81583700 & -1.11848200 & 0.00003900 \\ \mathrm{H} & -4.77111200 & -0.97334400 & 0.00003100 \\ \mathrm{H} & -1.60592600 & -2.09349100 & 0.00008800 \\ \mathrm{H} & 0.84303600 & -2.10181400 & 0.00009000 \\ \mathrm{H} & 0.85124600 & 2.18117400 & -0.00009500 \\ \mathrm{C} & 2.57256000 & -0.00903500 & 0.00000500 \\ \mathrm{C} & 3.05511800 & -0.75392600 & 1.25289800 \\ \mathrm{C} & 3.05513100 & -0.75406000 & -1.25280300 \\ \mathrm{C} & 3.19539600 & 1.38658800 & -0.00006100 \\ \mathrm{H} & 2.71980600 & -0.24505400 & 2.15730700 \\ \mathrm{H} & 2.68118900 & -1.77682400 & 1.28148600 \\ \mathrm{H} & 4.14553700 & -0.79318100 & 1.26408200 \\ \mathrm{H} & 2.71982100 & -0.24529100 & -2.15727000\end{array}$




$\begin{array}{lrrr}\mathrm{H} & 4.14555000 & -0.79330800 & -1.26397700 \\ \mathrm{H} & 2.68120900 & -1.77696400 & -1.28128100 \\ \mathrm{H} & 4.28192000 & 1.29641300 & 0.00000000 \\ \mathrm{H} & 2.90781700 & 1.95464100 & -0.88559000 \\ \mathrm{H} & 2.90772200 & 1.95476600 & 0.88535800\end{array}$

$\begin{array}{lrrr}\text { 4-Me } & & & \\ \text { C } & 0.01585300 & -1.18043900 & -0.00046100 \\ \text { C } & 0.69479800 & 0.03528100 & -0.00007300 \\ \text { C } & -0.02119700 & 1.22592400 & -0.00038400 \\ \text { C } & -1.40566400 & 1.20074200 & -0.00086500 \\ \text { C } & -2.09820700 & -0.00711500 & -0.00092100 \\ \text { C } & -1.36715300 & -1.19470100 & -0.00098400 \\ \text { C } & 2.17540700 & 0.10956600 & 0.00027900 \\ \text { O } & 2.81336200 & 1.12865700 & 0.00040300 \\ \text { H } & 0.52268400 & 2.16035600 & -0.00047100 \\ \text { O } & 2.76678400 & -1.10215000 & 0.00051500 \\ \text { H } & 3.72007700 & -0.94452100 & 0.00078200 \\ \text { H } & 0.57432800 & -2.10515500 & -0.00062500 \\ \text { C } & -3.60005100 & -0.04406700 & 0.00137000 \\ \text { H } & -3.97456200 & -0.59589800 & -0.86127400 \\ \text { H } & -3.97199100 & -0.54629100 & 0.89513400 \\ \text { H } & -4.02137700 & 0.95861300 & -0.02558100 \\ H & -1.89366300 & -2.14100400 & -0.00165800 \\ H & -1.95937100 & 2.13069400 & -0.00141400\end{array}$

$\begin{array}{lrrr}\text { 4-Et } & & & \\ \text { C } & 0.44913700 & -1.17941900 & -0.13040300 \\ \text { C } & 1.12309200 & 0.03464200 & -0.04760000 \\ \text { C } & 0.41218700 & 1.22829300 & -0.12123400 \\ \text { C } & -0.96185400 & 1.20435100 & -0.27595000 \\ \text { C } & -1.65085400 & -0.00466800 & -0.36117800 \\ \text { C } & -0.92709100 & -1.19217800 & -0.28542600 \\ \text { C } & 2.59490900 & 0.10731700 & 0.11461300 \\ \text { O } & 3.22947900 & 1.12587500 & 0.18866600 \\ \text { H } & 0.95480400 & 2.16135600 & -0.05828400 \\ \text { O } & 3.18247800 & -1.10477400 & 0.17450300 \\ \text { H } & 4.13008300 & -0.94749800 & 0.27896900 \\ \text { H } & 1.00430100 & -2.10457500 & -0.07626600 \\ H & -1.45118100 & -2.13756800 & -0.35324100 \\ H & -1.51360700 & 2.13438600 & -0.33646300 \\ \text { C } & -3.15036100 & -0.02574500 & -0.47416800 \\ \text { C } & -3.81341800 & -0.02705700 & 0.90548300 \\ \text { H } & -3.52564100 & 0.86019100 & 1.46949100 \\ H & -4.89911000 & -0.04134500 & 0.81762400 \\ \text { H } & -3.50305600 & -0.90081700 & 1.47852100 \\ \text { H } & -3.46156100 & -0.91081400 & -1.03055500 \\ H & -3.48518100 & 0.84465100 & -1.03996800\end{array}$




\begin{tabular}{|c|c|c|c|}
\hline \multicolumn{4}{|c|}{ 4-i-Pr } \\
\hline C & -0.69445000 & -1.09966200 & -0.00002100 \\
\hline C & -1.47416300 & 0.05377600 & 0.00000600 \\
\hline C & -0.86135600 & 1.30074200 & 0.00004800 \\
\hline C & 0.52032600 & 1.39123100 & 0.00006200 \\
\hline C & 1.31459500 & 0.24709000 & 0.00003500 \\
\hline C & 0.68534500 & -0.99794400 & -0.00000800 \\
\hline C & -2.95579100 & 0.00214600 & -0.00000600 \\
\hline $\mathrm{O}$ & -3.67794400 & 0.96341700 & 0.00000700 \\
\hline $\mathrm{H}$ & -1.48202700 & 2.18607500 & 0.00007100 \\
\hline $\mathrm{O}$ & -3.44241600 & -1.25535200 & -0.00003400 \\
\hline $\mathrm{H}$ & -4.40562500 & -1.17884300 & -0.00002100 \\
\hline $\mathrm{H}$ & -1.17283800 & -2.06829600 & -0.00005300 \\
\hline $\mathrm{H}$ & 1.28226100 & -1.90113300 & -0.00002800 \\
\hline $\mathrm{H}$ & 0.99370700 & 2.36492400 & 0.00009600 \\
\hline C & 2.82277700 & 0.35161700 & 0.00002700 \\
\hline $\mathrm{H}$ & 3.07328900 & 1.41459500 & 0.00010200 \\
\hline C & 3.42260300 & -0.27756100 & -1.25889500 \\
\hline $\mathrm{H}$ & 3.01004300 & 0.17370100 & -2.16065000 \\
\hline $\mathrm{H}$ & 4.50501300 & -0.14724600 & -1.27023100 \\
\hline $\mathrm{H}$ & 3.21423600 & -1.34802400 & -1.29200200 \\
\hline C & 3.42266300 & -0.27776100 & 1.25881800 \\
\hline $\mathrm{H}$ & 4.50507400 & -0.14745100 & 1.27011400 \\
\hline $\mathrm{H}$ & 3.01015500 & 0.17336100 & 2.16066700 \\
\hline $\mathrm{H}$ & 3.21430400 & -1.34822800 & 1.29176700 \\
\hline
\end{tabular}

$\begin{array}{lrrr}\text { 4-Cyclohexyl } & & \\ \mathrm{C} & 1.98892300 & 1.24450700 & 0.00026600 \\ \mathrm{C} & 2.53929900 & -0.03136700 & -0.00003700 \\ \mathrm{C} & 1.70330100 & -1.14464500 & -0.00032000 \\ \mathrm{C} & 0.33143400 & -0.97940700 & -0.00028800 \\ \mathrm{C} & -0.23799600 & 0.29599100 & 0.00002900 \\ \mathrm{C} & 0.61131800 & 1.39917200 & 0.00029400 \\ \mathrm{C} & 4.00435000 & -0.25654700 & -0.00005200 \\ \mathrm{O} & 4.53492600 & -1.33558100 & -0.00034200 \\ \mathrm{H} & 2.14976100 & -2.12940600 & -0.00054100 \\ \mathrm{O} & 4.71711400 & 0.88812900 & 0.00030200 \\ \mathrm{H} & 5.64903300 & 0.63304200 & 0.00025300 \\ \mathrm{H} & 2.63784000 & 2.10826900 & 0.00047300 \\ \mathrm{H} & 0.18552000 & 2.39458900 & 0.00053100 \\ \mathrm{H} & -0.30780500 & -1.85293800 & -0.00048400 \\ \mathrm{C} & -1.73522100 & 0.47495300 & 0.00012300 \\ \mathrm{C} & -2.38312600 & -0.11739200 & -1.25878600 \\ \mathrm{C} & -2.38300200 & -0.11832500 & 1.25865600 \\ \mathrm{H} & -1.94001700 & 1.55083400 & 0.00052600 \\ \mathrm{C} & -3.89463500 & 0.09676800 & -1.25781700 \\ \mathrm{H} & -2.17023400 & -1.19028400 & -1.29667200 \\ \mathrm{H} & -1.92999300 & 0.32565500 & -2.14720600 \\ \mathrm{C} & -3.89450900 & 0.09582800 & 1.25801000\end{array}$




$\begin{array}{lrrr}H & -2.17012000 & -1.19124900 & 1.29571000 \\ H & -1.92977200 & 0.32405000 & 2.14735700 \\ \mathrm{C} & -4.53401400 & -0.48634700 & -0.00009100 \\ \mathrm{H} & -4.33544300 & -0.34790600 & -2.15093000 \\ \mathrm{H} & -4.10448000 & 1.17006300 & -1.30234400 \\ \mathrm{H} & -4.33522700 & -0.34952700 & 2.15083300 \\ \mathrm{H} & -4.10436800 & 1.16908300 & 1.30337200 \\ \mathrm{H} & -5.60888000 & -0.30120400 & 0.00003000 \\ \mathrm{H} & -4.39886500 & -1.57259100 & -0.00050400\end{array}$

\begin{tabular}{lrrr}
\multicolumn{4}{l}{ 4-Cyclobutyl } \\
C & 1.15375200 & 1.17495500 & -0.10664700 \\
$\mathrm{C}$ & 1.83594200 & -0.03523800 & -0.03386200 \\
$\mathrm{C}$ & 1.13142800 & -1.23316700 & -0.10285100 \\
$\mathrm{C}$ & -0.24399100 & -1.21801400 & -0.24551000 \\
$\mathrm{C}$ & -0.93936900 & -0.01205000 & -0.32467000 \\
$\mathrm{C}$ & -0.22388000 & 1.18057000 & -0.24952300 \\
$\mathrm{C}$ & 3.30859000 & -0.09915800 & 0.12217100 \\
$\mathrm{O}$ & 3.95015400 & -1.11378800 & 0.19137200 \\
$\mathrm{H}$ & 1.67971500 & -2.16291100 & -0.03949800 \\
$\mathrm{O}$ & 3.88934500 & 1.11645500 & 0.18111500 \\
$\mathrm{H}$ & 4.83832000 & 0.96431800 & 0.28043400 \\
$\mathrm{H}$ & 1.70329300 & 2.10321600 & -0.04788700 \\
$\mathrm{H}$ & -0.75523800 & 2.12259700 & -0.29944900 \\
$\mathrm{H}$ & -0.79175200 & -2.15080300 & -0.29214800 \\
$\mathrm{C}$ & -2.42701700 & -0.00070200 & -0.49776000 \\
$\mathrm{C}$ & -3.28979600 & -1.05650800 & 0.23120600 \\
$\mathrm{C}$ & -3.27094500 & 1.09397800 & 0.19414000 \\
$\mathrm{H}$ & -2.66678200 & -0.01745800 & -1.56428200 \\
$\mathrm{C}$ & -4.36887600 & 0.03171000 & 0.41302800 \\
$\mathrm{H}$ & -2.83411000 & -1.32363500 & 1.18515000 \\
$\mathrm{H}$ & -3.55710800 & -1.96371500 & -0.30622500 \\
$\mathrm{H}$ & -2.81082600 & 1.38525000 & 1.13890600 \\
$\mathrm{H}$ & -3.52245300 & 1.98702500 & -0.37385500 \\
$\mathrm{H}$ & -4.91471800 & 0.05282700 & 1.35271100 \\
$\mathrm{H}$ & -5.07937100 & 0.02370300 & -0.41208300
\end{tabular}

\section{4-Cyclopentyl}

$\begin{array}{lrrr}\text { C } & 1.67364200 & 1.26210300 & 0.13912700 \\ \text { C } & 2.15786300 & -0.03235600 & 0.00021200 \\ \text { C } & 1.26628400 & -1.10021900 & -0.07608700 \\ \text { C } & -0.09333000 & -0.86878000 & -0.01432300 \\ \text { C } & -0.59681400 & 0.42707400 & 0.12512000 \\ \text { C } & 0.30579000 & 1.48303000 & 0.20087300 \\ \text { C } & 3.60859100 & -0.32698100 & -0.06918300 \\ \text { O } & 4.08283900 & -1.42561400 & -0.18823400 \\ \text { H } & 1.66200100 & -2.10070800 & -0.18301100 \\ \text { O } & 4.37919300 & 0.77641800 & 0.01504300 \\ \text { H } & 5.29629700 & 0.47729400 & -0.03959000\end{array}$




$\begin{array}{lrrr}\mathrm{H} & 2.36574000 & 2.08940800 & 0.19976800 \\ \mathrm{H} & -0.06865200 & 2.49276300 & 0.31139200 \\ \mathrm{H} & -0.78136300 & -1.70402400 & -0.07318400 \\ \mathrm{C} & -2.08197900 & 0.66805500 & 0.16870500 \\ \mathrm{C} & -2.78412900 & 0.34967300 & -1.17085700 \\ \mathrm{C} & -2.84785400 & -0.18207300 & 1.22736900 \\ \mathrm{H} & -2.24157900 & 1.72403500 & 0.39003600 \\ \mathrm{C} & -4.20699900 & -0.02222500 & -0.76370100 \\ \mathrm{H} & -2.30365500 & -0.51332300 & -1.63786000 \\ \mathrm{H} & -2.72229300 & 1.17987800 & -1.87290500 \\ \mathrm{C} & -3.97272300 & -0.90166300 & 0.46448700 \\ \mathrm{H} & -3.26490700 & 0.47643000 & 1.98850400 \\ \mathrm{H} & -2.18644600 & -0.87832300 & 1.73999800 \\ \mathrm{H} & -4.76162900 & 0.87588000 & -0.48042800 \\ \mathrm{H} & -4.76285300 & -0.52329900 & -1.55475200 \\ \mathrm{H} & -4.86484800 & -1.04973500 & 1.07046700 \\ \mathrm{H} & -3.63212400 & -1.88653700 & 0.13663600\end{array}$

$\begin{array}{lrrr}\text { 4-Bn } & & & \\ \mathrm{C} & 1.62370000 & -0.79160000 & 0.64870000 \\ \mathrm{C} & 2.61560000 & -0.02240000 & 0.04610000 \\ \mathrm{C} & 2.31650000 & 1.25330000 & -0.41620000 \\ \mathrm{C} & 1.03430000 & 1.75620000 & -0.27310000 \\ \mathrm{C} & 0.03460000 & 0.99840000 & 0.33060000 \\ \mathrm{C} & 0.34460000 & -0.28240000 & 0.78460000 \\ \mathrm{C} & 4.00090000 & -0.52190000 & -0.12990000 \\ \mathrm{O} & 4.89410000 & 0.09580000 & -0.64560000 \\ \mathrm{H} & 3.09800000 & 1.83370000 & -0.88660000 \\ \mathrm{O} & 4.18040000 & -1.76730000 & 0.35350000 \\ \mathrm{H} & 5.10500000 & -1.99640000 & 0.19160000 \\ \mathrm{H} & 1.85850000 & -1.78450000 & 1.00370000 \\ \mathrm{H} & -0.43050000 & -0.88620000 & 1.23940000 \\ \mathrm{H} & 0.80330000 & 2.74980000 & -0.63570000 \\ \mathrm{C} & -1.35730000 & 1.56050000 & 0.51160000 \\ \mathrm{H} & -1.45250000 & 2.46530000 & -0.09150000 \\ \mathrm{C} & -2.45430000 & 0.58970000 & 0.14680000 \\ \mathrm{C} & -2.53480000 & 0.08330000 & -1.14900000 \\ \mathrm{C} & -3.39280000 & 0.17630000 & 1.08410000 \\ \mathrm{C} & -3.53370000 & -0.80970000 & -1.49940000 \\ \mathrm{H} & -1.80030000 & 0.39000000 & -1.88430000 \\ \mathrm{C} & -4.39590000 & -0.72150000 & 0.73780000 \\ \mathrm{H} & -3.33950000 & 0.56020000 & 2.09550000 \\ \mathrm{C} & -4.46910000 & -1.21580000 & -0.55460000 \\ \mathrm{H} & -3.58370000 & -1.19220000 & -2.50990000 \\ \mathrm{H} & -5.11800000 & -1.03330000 & 1.48010000 \\ \mathrm{H} & -5.24820000 & -1.91440000 & -0.82690000 \\ \mathrm{H} & -1.48230000 & 1.86610000 & 1.55290000\end{array}$

\section{4-SiMe ${ }_{3}$}




$\begin{array}{lrrr}\text { C } & -1.42258600 & -1.16615800 & -0.00000800 \\ \mathrm{C} & -2.11392800 & 0.04164500 & -0.00000800 \\ \mathrm{C} & -1.41563800 & 1.24302500 & -0.00003000 \\ \mathrm{C} & -0.02976500 & 1.23437500 & -0.00005600 \\ \mathrm{C} & 0.68770200 & 0.03575500 & -0.00004900 \\ \mathrm{C} & -0.03742900 & -1.15974900 & -0.00002600 \\ \mathrm{C} & -3.59788900 & 0.09669800 & 0.00001400 \\ \mathrm{O} & -4.24734300 & 1.10797300 & 0.00004200 \\ \mathrm{H} & -1.97382600 & 2.16916200 & -0.00003100 \\ \mathrm{O} & -4.17293600 & -1.12205300 & -0.00000200 \\ \mathrm{H} & -5.12844200 & -0.97797300 & 0.00001400 \\ \mathrm{H} & -1.97076100 & -2.09716200 & 0.00000300 \\ \mathrm{H} & 0.48683700 & -2.10901700 & -0.00002800 \\ \mathrm{H} & 0.49891600 & 2.17960400 & -0.00008800 \\ \mathrm{Si} & 2.57510800 & -0.00705000 & -0.00000200 \\ \mathrm{C} & 3.15182800 & -0.92240200 & -1.53314300 \\ \mathrm{H} & 4.23997800 & -0.99873800 & -1.55219600 \\ \mathrm{H} & 2.74453400 & -1.93399600 & -1.56074300 \\ \mathrm{H} & 2.83035100 & -0.40724800 & -2.43866200 \\ \mathrm{C} & 3.15181300 & -0.92069600 & 1.53416800 \\ \mathrm{H} & 4.23996800 & -0.99690100 & 1.55340700 \\ \mathrm{H} & 2.83020400 & -0.40456800 & 2.43908700 \\ \mathrm{H} & 2.74460200 & -1.93229300 & 1.56284900 \\ \mathrm{C} & 3.23237100 & 1.74845800 & -0.00094500 \\ \mathrm{H} & 2.90306400 & 2.29627800 & -0.88483100 \\ \mathrm{H} & 2.90323000 & 2.29716300 & 0.88245600 \\ \mathrm{H} & 4.32318900 & 1.74133500 & -0.00103400\end{array}$

$\begin{array}{lrrr}\text { 4-OPh } & & & \\ \text { C } & -2.33425800 & 1.24051800 & -0.31565900 \\ \text { C } & -2.61359600 & -0.07240100 & 0.05805700 \\ \text { C } & -1.57237000 & -0.92217000 & 0.41405200 \\ \text { C } & -0.26181700 & -0.48078900 & 0.39636500 \\ \text { C } & 0.00456800 & 0.83108200 & 0.01410600 \\ \text { C } & -1.02996100 & 1.69241900 & -0.33919100 \\ \text { C } & -3.99342100 & -0.60414600 & 0.09488300 \\ \text { O } & -4.29166900 & -1.72726700 & 0.40553100 \\ \text { H } & -1.80780700 & -1.93509400 & 0.70989600 \\ \text { O } & -4.92209800 & 0.30711400 & -0.26124000 \\ \text { H } & -5.77903400 & -0.13549400 & -0.20420900 \\ \text { H } & -3.14259200 & 1.90226700 & -0.59034100 \\ \text { H } & -0.78683600 & 2.70542400 & -0.62587200 \\ \text { H } & 0.54472800 & -1.14085800 & 0.67960000 \\ \text { O } & 1.25863300 & 1.36723700 & 0.00390800 \\ \text { C } & 2.35843900 & 0.53364400 & -0.03043300 \\ \text { C } & 3.30411700 & 0.66344400 & 0.97143900 \\ \text { C } & 2.53991500 & -0.35712600 & -1.07851700 \\ \text { C } & 4.45282700 & -0.11491500 & 0.92343000 \\ \text { H } & 3.13128800 & 1.37239700 & 1.76875700\end{array}$




$\begin{array}{llll}\mathrm{C} & 3.68707400 & -1.13413100 & -1.11220400 \\ \mathrm{H} & 1.78648000 & -0.43322600 & -1.85094900 \\ \mathrm{C} & 4.64565600 & -1.01602300 & -0.11329600 \\ \mathrm{H} & 5.19654500 & -0.01618900 & 1.70202500 \\ \mathrm{H} & 3.83541200 & -1.83011400 & -1.92630600 \\ \mathrm{H} & 5.53985900 & -1.62221800 & -0.14637200\end{array}$

\begin{tabular}{|c|c|c|c|}
\hline \multicolumn{4}{|c|}{ 4-Ph } \\
\hline C & 1.67237800 & -1.13263600 & 0.31441200 \\
\hline C & 2.35904600 & 0.03305500 & -0.00857200 \\
\hline C & 1.65142500 & 1.18702600 & -0.32892200 \\
\hline C & 0.26944300 & 1.17462900 & -0.32556000 \\
\hline C & -0.43348400 & 0.01134400 & -0.00361100 \\
\hline C & 0.28842700 & -1.13950100 & 0.31540700 \\
\hline C & 3.84081600 & 0.09438100 & -0.02522000 \\
\hline $\mathrm{O}$ & 4.48451100 & 1.07253800 & -0.29694300 \\
\hline $\mathrm{H}$ & 2.20266600 & 2.08093600 & -0.58564200 \\
\hline $\mathrm{O}$ & 4.42278200 & -1.07687800 & 0.30032900 \\
\hline $\mathrm{H}$ & 5.37744100 & -0.93272500 & 0.26075000 \\
\hline $\mathrm{H}$ & 2.22381200 & -2.02531100 & 0.57119900 \\
\hline $\mathrm{H}$ & -0.24228700 & -2.04088000 & 0.59067600 \\
\hline $\mathrm{H}$ & -0.27633300 & 2.06759900 & -0.59870700 \\
\hline C & -1.91328700 & -0.00036200 & -0.00064300 \\
\hline C & -2.63278500 & 1.09778100 & 0.47012400 \\
\hline C & -2.61762000 & -1.10959000 & -0.46833900 \\
\hline C & -4.01885000 & 1.08638700 & 0.47532500 \\
\hline $\mathrm{H}$ & -2.10056700 & 1.95638400 & 0.85798000 \\
\hline C & -4.00369300 & -1.12004400 & -0.46720900 \\
\hline $\mathrm{H}$ & -2.07370100 & -1.95949600 & -0.85914600 \\
\hline C & -4.70914700 & -0.02232300 & 0.00571800 \\
\hline $\mathrm{H}$ & -4.56097300 & 1.94242200 & 0.85323400 \\
\hline $\mathrm{H}$ & -4.53402800 & -1.98436800 & -0.84298600 \\
\hline $\mathrm{H}$ & -5.79038900 & -0.03073000 & 0.00808500 \\
\hline
\end{tabular}

$\begin{array}{crrr}\text { 4-F } & & & \\ \text { C } & -0.02419300 & -1.18621600 & 0.00000600 \\ \text { C } & 0.64653300 & 0.03313600 & 0.00000300 \\ \text { C } & -0.07009400 & 1.22634300 & -0.00000600 \\ \text { C } & -1.45265600 & 1.20957700 & -0.00001400 \\ \text { C } & -2.09278900 & -0.01675200 & -0.00001100 \\ \text { C } & -1.40848900 & -1.21753200 & -0.00000100 \\ \text { C } & 2.12719200 & 0.11305200 & 0.00001100 \\ \text { O } & 2.75798600 & 1.13593600 & 0.00000900 \\ \text { H } & 0.47468000 & 2.15990100 & -0.00000800 \\ \text { O } & 2.72118000 & -1.09608900 & 0.00002000 \\ H & 3.67462700 & -0.93852200 & 0.00002400 \\ H & 0.53959700 & -2.10729900 & 0.00001300 \\ H & -1.96052200 & -2.14590900 & 0.00000100 \\ H & -2.03835900 & 2.11710000 & -0.00002200\end{array}$




$\begin{array}{lrrr}F & -3.43070800 & -0.04152100 & -0.00001800 \\ 4-N_{3} & & & \\ \text { C } & 0.86010700 & -1.28856700 & -0.00002400 \\ \text { C } & 1.33982500 & 0.01921800 & -0.00000900 \\ \mathrm{C} & 0.44490800 & 1.08370500 & 0.00001900 \\ \mathrm{C} & -0.91735800 & 0.85310500 & 0.00003700 \\ \mathrm{C} & -1.39025200 & -0.45856200 & 0.00001500 \\ \mathrm{C} & -0.49957900 & -1.52892700 & -0.00001100 \\ \mathrm{C} & 2.78869100 & 0.32737300 & -0.00000800 \\ \mathrm{O} & 3.25444200 & 1.43568400 & -0.00000700 \\ \mathrm{H} & 0.83597100 & 2.09158600 & 0.00003400 \\ \mathrm{O} & 3.56313000 & -0.77537800 & -0.00000300 \\ \mathrm{H} & 4.48036900 & -0.47128100 & 0.00000000 \\ \mathrm{H} & 1.55693500 & -2.11380900 & -0.00004300 \\ \mathrm{H} & -0.89326600 & -2.53501800 & -0.00001000 \\ \mathrm{H} & -1.60708800 & 1.68690200 & 0.00006900 \\ \mathrm{~N} & -2.76361900 & -0.79575200 & 0.00007100 \\ \mathrm{~N} & -3.55523200 & 0.14899000 & -0.00002900 \\ \mathrm{~N} & -4.34851200 & 0.93463300 & -0.00005400\end{array}$

$\begin{array}{lrrr}\text { 4-Si(OMe) }{ }_{3} & & & \\ \mathrm{C} & -2.23920900 & -1.17479100 & 0.00807900 \\ \mathrm{C} & -2.92180100 & 0.03666600 & 0.00203100 \\ \mathrm{C} & -2.22017400 & 1.23743800 & 0.01223700 \\ \mathrm{C} & -0.83625600 & 1.22370700 & 0.02534300 \\ \mathrm{C} & -0.13345200 & 0.01640700 & 0.02583200 \\ \mathrm{C} & -0.85300700 & -1.17946700 & 0.02089000 \\ \mathrm{C} & -4.40666100 & 0.09898700 & -0.01040700 \\ \mathrm{O} & -5.05019400 & 1.11379000 & -0.01384200 \\ \mathrm{H} & -2.77539100 & 2.16506000 & 0.01309300 \\ \mathrm{O} & -4.98758400 & -1.11649600 & -0.01809200 \\ \mathrm{H} & -5.94236100 & -0.96785900 & -0.02634800 \\ \mathrm{H} & -2.79324500 & -2.10208400 & 0.00581400 \\ \mathrm{H} & -0.32330600 & -2.12332800 & 0.03599800 \\ \mathrm{H} & -0.29513800 & 2.16115000 & 0.04472600 \\ \mathrm{Si} & 1.71629400 & 0.00297600 & 0.00209200 \\ \mathrm{O} & 2.26688200 & 1.20785400 & 0.96663200 \\ \mathrm{O} & 2.26370100 & 0.24627300 & -1.52395800 \\ \mathrm{O} & 2.23750100 & -1.45554500 & 0.53956500 \\ \mathrm{C} & 3.47628800 & 1.90676100 & 0.74664900 \\ \mathrm{H} & 3.55612000 & 2.23611100 & -0.29137200 \\ \mathrm{H} & 3.48994600 & 2.77937500 & 1.39639300 \\ \mathrm{H} & 4.34128200 & 1.28186200 & 0.98672500 \\ \mathrm{C} & 3.42428500 & -1.65094500 & 1.28231300 \\ \mathrm{H} & 3.49715300 & -0.93275600 & 2.10166100 \\ \mathrm{H} & 3.40818400 & -2.65689900 & 1.69654900 \\ \mathrm{H} & 4.30774200 & -1.55162600 & 0.64510000 \\ \mathrm{C} & 3.44251900 & -0.32682900 & -2.05170700\end{array}$




$\begin{array}{rrrr}H & 3.43031100 & -0.20151200 & -3.13246600 \\ H & 4.33335500 & 0.16882400 & -1.65472600 \\ H & 3.49958700 & -1.39261100 & -1.82041900\end{array}$

$\begin{array}{lrrr}\text { 4-Cl } & & & \\ \mathrm{C} & -0.40137800 & -1.18092300 & -0.00000800 \\ \mathrm{C} & -1.07815000 & 0.03405600 & -0.00000400 \\ \mathrm{C} & -0.36649700 & 1.22915800 & 0.00000400 \\ \mathrm{C} & 1.01631100 & 1.21673300 & 0.00001000 \\ \mathrm{C} & 1.67700800 & -0.00367400 & 0.00000500 \\ \mathrm{C} & 0.98328300 & -1.20404800 & -0.00000300 \\ \mathrm{C} & -2.56071200 & 0.10715300 & -0.00000900 \\ \mathrm{O} & -3.19456100 & 1.12770600 & -0.00000600 \\ \mathrm{H} & -0.91322900 & 2.16169600 & 0.00000800 \\ \mathrm{O} & -3.14803600 & -1.10447900 & -0.00001700 \\ \mathrm{H} & -4.10255400 & -0.95306000 & -0.00002000 \\ \mathrm{H} & -0.95876100 & -2.10603000 & -0.00001400 \\ \mathrm{H} & 1.52540100 & -2.13809300 & -0.00000600 \\ \mathrm{H} & 1.58416000 & 2.13536300 & 0.00001700 \\ \mathrm{Cl} & 3.41097500 & -0.02802500 & 0.00001400\end{array}$

$\begin{array}{lrrr}\text { 4-Br } & & & \\ \mathrm{C} & -1.08086200 & -1.17814400 & -0.00001000 \\ \mathrm{C} & -1.76098700 & 0.03467400 & -0.00000600 \\ \mathrm{C} & -1.05313100 & 1.23171900 & 0.00000300 \\ \mathrm{C} & 0.33033200 & 1.22383500 & 0.00000700 \\ \mathrm{C} & 0.99439000 & 0.00511500 & 0.00000300 \\ \mathrm{C} & 0.30449000 & -1.19763200 & -0.00000600 \\ \mathrm{C} & -3.24420100 & 0.10341400 & -0.00001000 \\ \mathrm{O} & -3.88061600 & 1.12224900 & -0.00000700 \\ \mathrm{H} & -1.60244700 & 2.16282000 & 0.00000600 \\ \mathrm{O} & -3.82770300 & -1.10986500 & -0.00001800 \\ \mathrm{H} & -4.78274700 & -0.96167200 & -0.00002000 \\ \mathrm{H} & -1.63533600 & -2.10508200 & -0.00001600 \\ \mathrm{H} & 0.84429300 & -2.13290900 & -0.00000900 \\ \mathrm{H} & 0.89042900 & 2.14708700 & 0.00001400 \\ \mathrm{Br} & 2.88606200 & -0.01563400 & 0.00000900\end{array}$

$\begin{array}{lrrr}\mathbf{4 - F _ { 5 } \mathbf { P h }} & & & \\ \mathrm{C} & 2.75447900 & 0.92016900 & -0.73298600 \\ \mathrm{C} & 3.43958000 & -0.02719600 & 0.01904900 \\ \mathrm{C} & 2.73715600 & -0.96657500 & 0.76534000 \\ \mathrm{C} & 1.35506600 & -0.96115800 & 0.75892800 \\ \mathrm{C} & 0.66015900 & -0.01240100 & 0.00881800 \\ \mathrm{C} & 1.37046200 & 0.92763100 & -0.73533700 \\ \mathrm{C} & 4.92475400 & -0.07558400 & 0.05764500 \\ \mathrm{O} & 5.56481700 & -0.86870800 & 0.69292200 \\ \mathrm{H} & 3.29032900 & -1.69203300 & 1.34499100 \\ \mathrm{O} & 5.50203200 & 0.87327500 & -0.70237600\end{array}$




$\begin{array}{lrrr}H & 6.45795700 & 0.76185000 & -0.61301700 \\ H & 3.30495600 & 1.64582300 & -1.31320700 \\ H & 0.83755600 & 1.66165700 & -1.32286500 \\ H & 0.80992300 & -1.68933400 & 1.34248400 \\ \mathrm{C} & -0.82088400 & -0.00399600 & 0.00341600 \\ \mathrm{C} & -1.55928500 & -1.16918000 & -0.17618000 \\ \mathrm{C} & -1.54776600 & 1.16918000 & 0.17787700 \\ \mathrm{C} & -2.94231700 & -1.17456000 & -0.18498000 \\ \mathrm{C} & -2.93065200 & 1.18966000 & 0.17732800 \\ \mathrm{C} & -3.63192000 & 0.01135000 & -0.00617500 \\ \mathrm{~F} & -0.94079600 & -2.33098700 & -0.37361600 \\ \mathrm{~F} & -3.60950100 & -2.30474600 & -0.37221800 \\ \mathrm{~F} & -4.95477200 & 0.01863700 & -0.01079600 \\ \mathrm{~F} & -3.58669700 & 2.32721500 & 0.35977000 \\ \mathrm{~F} & -0.91806800 & 2.32448800 & 0.37917000\end{array}$

\section{4-OAC}

$\begin{array}{lrrr}\text { C } & 1.05592700 & 1.18697100 & -0.20552700 \\ \mathrm{C} & 1.71964100 & -0.03103900 & -0.09769700 \\ \mathrm{C} & 1.01814500 & -1.22497300 & -0.23502300 \\ \mathrm{C} & -0.34392200 & -1.20599900 & -0.47283500 \\ \mathrm{C} & -0.99688000 & 0.01495900 & -0.56505600 \\ \mathrm{C} & -0.30824900 & 1.21194200 & -0.44246400 \\ \mathrm{C} & 3.18017500 & -0.10902500 & 0.15723400 \\ \mathrm{O} & 3.80138200 & -1.13187200 & 0.26149400 \\ \mathrm{H} & 1.55846700 & -2.15789200 & -0.15599600 \\ \mathrm{O} & 3.76121400 & 1.10056300 & 0.26546000 \\ \mathrm{H} & 4.70144100 & 0.94665800 & 0.42806600 \\ \mathrm{H} & 1.60918100 & 2.10927100 & -0.10680100 \\ \mathrm{H} & -0.84716400 & 2.14403600 & -0.54106000 \\ \mathrm{H} & -0.90965900 & -2.11927600 & -0.59535300 \\ \mathrm{O} & -2.34604500 & 0.04086400 & -0.84744300 \\ \mathrm{C} & -2.77441500 & -0.03898700 & 1.56183500 \\ \mathrm{H} & -2.17298800 & -0.93412900 & 1.71583900 \\ \mathrm{H} & -2.13554200 & 0.81904000 & 1.76763600 \\ \mathrm{H} & -3.62767800 & -0.04158100 & 2.23031200 \\ \mathrm{C} & -3.28708200 & 0.01275200 & 0.14943000 \\ \mathrm{O} & -4.43606300 & 0.03222700 & -0.15976400\end{array}$

\section{4-CHO}

C

C

C

C

C

C

C

$\mathrm{O}$

$\mathrm{H}$

$$
\begin{array}{rrr}
0.26286600 & -1.12136900 & -0.00000300 \\
1.02199900 & 0.04827800 & -0.00000400 \\
0.40632000 & 1.29427500 & 0.00000000 \\
-0.97597400 & 1.37048900 & 0.00000600 \\
-1.73587000 & 0.20628100 & 0.00000700 \\
-1.11684100 & -1.04078300 & 0.00000300 \\
2.51091300 & 0.01391600 & -0.00001000 \\
3.21324300 & 0.98768400 & -0.00001100 \\
1.02356900 & 2.18139000 & -0.00000100
\end{array}
$$




$\begin{array}{lrrr}\mathrm{O} & 3.00761500 & -1.23561500 & -0.00001400 \\ \mathrm{H} & 3.97104900 & -1.15678500 & -0.00001700 \\ \mathrm{H} & 0.75987600 & -2.08013700 & -0.00000600 \\ \mathrm{H} & -1.73429400 & -1.92855800 & 0.00000400 \\ \mathrm{H} & -1.47126000 & 2.33371800 & 0.00000900 \\ \mathrm{C} & -3.21681300 & 0.30418500 & 0.00001300 \\ \mathrm{O} & -3.95477600 & -0.64405300 & 0.00001500 \\ \mathrm{H} & -3.61719400 & 1.33461100 & 0.00001600\end{array}$

\section{4-COPh}

C

$\begin{array}{lll}2.44595100 & 1.11216900 & -0.48863700\end{array}$

C $\quad 2.78015300 \quad-0.12335000 \quad 0.05901500$

C $\quad 1.79805900 \quad-0.93458600 \quad 0.61525800$

C $\quad 0.47697400 \quad-0.52145000 \quad 0.60715500$

C $\quad 0.13630900 \quad 0.71308500 \quad 0.05867200$

C $\quad \begin{array}{llll}\text { C } & 1.12781800 & 1.53134000 & -0.47614400\end{array}$

C $\quad 4.18257400 \quad-0.61923200 \quad 0.07850000$

$\begin{array}{lllll}\mathrm{O} & 4.52644900 & -1.67438100 & 0.53788900\end{array}$

H $\quad 2.08764100 \quad-1.88202100 \quad 1.04744300$

$\begin{array}{lllll}\mathrm{O} & 5.05333900 & 0.24156800 & -0.47931600\end{array}$

H $\quad 5.92808600 \quad-0.16503300 \quad-0.41889300$

$\begin{array}{llll}H & 3.21796700 & 1.73651400 & -0.91355800\end{array}$

$\begin{array}{llll}\mathrm{H} & 0.84615900 & 2.49585300 & -0.87485300\end{array}$

$\mathrm{H} \quad-0.28764000 \quad-1.14830400 \quad 1.04470000$

C $\quad-1.26840600 \quad 1.23982300 \quad 0.08884300$

$\begin{array}{lllll}\text { O } & -1.46075100 & 2.42968400 & 0.19463400\end{array}$

$\begin{array}{llll}\text { C } & -2.41740900 & 0.28895500 & -0.00886600\end{array}$

$\begin{array}{llll}\text { C } & -2.33178200 & -0.91212700 & -0.70847700\end{array}$

C $\quad-3.62741300 \quad 0.66484100 \quad 0.57076000$

C $\quad-3.44614500-1.73052000-0.82304700$

H $\quad-1.40247900-1.19779500 \quad-1.18230900$

C $\quad-4.73231700 \quad-0.16276500 \quad 0.47353300$

$\begin{array}{llll}\mathrm{H} & -3.68042800 & 1.61093000 & 1.09164100\end{array}$

C $\quad-4.64238000-1.36124200-0.22496800$

H $\quad-3.37998700 \quad-2.65527900 \quad-1.37918400$

H $\quad-5.66597300 \quad 0.12562500 \quad 0.93624400$

H $\quad-5.50756700 \quad-2.00510000 \quad-0.30647300$

\section{4- $\mathrm{CO}_{2} \mathrm{Ph}$}

$\begin{array}{lrrr}C & -2.91284700 & 1.20310900 & 0.00201300 \\ C & -3.40423800 & -0.09889100 & -0.00015900 \\ C & -2.53266400 & -1.18247900 & -0.00189200 \\ C & -1.16580700 & -0.96842800 & -0.00147000 \\ C & -0.67403100 & 0.33364500 & 0.00070200 \\ C & -1.54591200 & 1.41705700 & 0.00244700 \\ C & -4.86519400 & -0.38452500 & -0.00072200 \\ O & -5.34376800 & -1.48579500 & -0.00261000 \\ H & -2.94412700 & -2.18185700 & -0.00354900 \\ O & -5.61907300 & 0.72960900 & 0.00111600\end{array}$




$\begin{array}{lrrr}H & -6.54261500 & 0.44447300 & 0.00057900 \\ \mathrm{H} & -3.59978900 & 2.03623100 & 0.00333800 \\ \mathrm{H} & -1.13442400 & 2.41638200 & 0.00411200 \\ \mathrm{H} & -0.47907200 & -1.80151300 & -0.00280700 \\ \mathrm{C} & 0.78794600 & 0.62801900 & 0.00124500 \\ \mathrm{O} & 1.25814500 & 1.72819500 & 0.00324700 \\ \mathrm{O} & 1.53053600 & -0.50413900 & -0.00079900 \\ \mathrm{C} & 2.91672500 & -0.35240300 & -0.00062800 \\ \mathrm{C} & 3.58685200 & -0.30850600 & 1.20694100 \\ \mathrm{C} & 3.58641500 & -0.30004100 & -1.20812200 \\ \mathrm{C} & 4.97072100 & -0.21272900 & 1.20141500 \\ \mathrm{H} & 3.02530500 & -0.34845000 & 2.12974400 \\ \mathrm{C} & 4.97026400 & -0.20428400 & -1.20242800 \\ \mathrm{H} & 3.02452800 & -0.33344900 & -2.13097800 \\ \mathrm{C} & 5.66309600 & -0.16097900 & -0.00046400 \\ \mathrm{H} & 5.50743400 & -0.17732000 & 2.13920100 \\ \mathrm{H} & 5.50663400 & -0.16225800 & -2.14013900 \\ \mathrm{H} & 6.74145500 & -0.08659200 & -0.00040700\end{array}$

$\begin{array}{lrrr}4-\mathrm{CO}_{2} \mathrm{Me} & & & \\ \mathrm{C} & 1.22816500 & 1.20896200 & 0.00018000 \\ \mathrm{C} & 1.81363100 & -0.05345800 & -0.00001800 \\ \mathrm{C} & 1.02324200 & -1.19778400 & -0.00019500 \\ \mathrm{C} & -0.35543300 & -1.08354400 & -0.00017500 \\ \mathrm{C} & -0.94165300 & 0.17882000 & 0.00002400 \\ \mathrm{C} & -0.15098200 & 1.32253100 & 0.00020100 \\ \mathrm{C} & 3.29119900 & -0.23094400 & -0.00004700 \\ \mathrm{O} & 3.85017900 & -1.29384200 & -0.00019900 \\ \mathrm{H} & 1.50643900 & -2.16452500 & -0.00034800 \\ \mathrm{O} & 3.96152600 & 0.93566000 & 0.00011500 \\ \mathrm{H} & 4.90337700 & 0.71864000 & 0.00008300 \\ \mathrm{H} & 1.85261600 & 2.08993700 & 0.00031700 \\ \mathrm{H} & -0.63432700 & 2.28913300 & 0.00035700 \\ \mathrm{H} & -0.98025000 & -1.96402600 & -0.00031200 \\ \mathrm{C} & -2.42295600 & 0.36026800 & 0.00006800 \\ \mathrm{O} & -2.97034900 & 1.43069500 & 0.00024500 \\ \mathrm{O} & -3.08436900 & -0.80237400 & -0.00011300 \\ \mathrm{C} & -4.50750000 & -0.69047900 & -0.00008100 \\ \mathrm{H} & -4.84232700 & -0.15419300 & -0.88556900 \\ \mathrm{H} & -4.88534000 & -1.70712700 & -0.00064300 \\ \mathrm{H} & -4.84235700 & -0.15518100 & 0.88599300\end{array}$

\section{$4-\mathrm{CO}_{2} \mathrm{H}$}

$\mathrm{C}$

C $\quad-1.38096400 \quad-0.03347800 \quad-0.00000600$

$\begin{array}{lll}-0.72164000 & 1.19221500 & -0.00000400\end{array}$

C $\quad-0.66150700 \quad-1.22339300-0.00000200$

C $\quad 0.72164500 \quad-1.19224700 \quad 0.00000400$

C $\quad 1.38097600 \quad 0.03344400 \quad 0.00000600$

$\begin{array}{lllll}\text { C } & 0.66151200 & 1.22335400 & 0.00000100\end{array}$ 


$\begin{array}{lrrr}\mathrm{C} & -2.86724200 & -0.12277700 & -0.00001200 \\ \mathrm{O} & -3.48714000 & -1.15103800 & -0.00001400 \\ \mathrm{H} & -1.20218200 & -2.15914300 & -0.00000300 \\ \mathrm{O} & -3.46632000 & 1.08145200 & -0.00001500 \\ \mathrm{H} & -4.41968200 & 0.92207600 & -0.00001900 \\ \mathrm{H} & -1.29259100 & 2.10867900 & -0.00000800 \\ \mathrm{H} & 1.20220000 & 2.15909700 & 0.00000200 \\ \mathrm{H} & 1.29259800 & -2.10870900 & 0.00000700 \\ \mathrm{C} & 2.86722900 & 0.12279600 & 0.00001200 \\ \mathrm{O} & 3.48711100 & 1.15106000 & 0.00001200 \\ \mathrm{O} & 3.46633800 & -1.08141600 & 0.00001600 \\ \mathrm{H} & 4.41968500 & -0.92195300 & 0.00002100\end{array}$

$\begin{array}{lrrr}4-\mathrm{CCl}_{3} & & & \\ \mathrm{C} & 1.69059800 & -1.14490900 & -0.00014600 \\ \mathrm{C} & 2.39105900 & 0.05928100 & -0.00010700 \\ \mathrm{C} & 1.70192900 & 1.26129600 & -0.00012400 \\ \mathrm{C} & 0.31461500 & 1.27245000 & -0.00018000 \\ \mathrm{C} & -0.38405300 & 0.07431400 & -0.00024100 \\ \mathrm{C} & 0.31076600 & -1.13493200 & -0.00020900 \\ \mathrm{C} & 3.87761500 & 0.10512600 & -0.00001300 \\ \mathrm{O} & 4.52817300 & 1.11420400 & 0.00000300 \\ \mathrm{H} & 2.26239900 & 2.18546900 & -0.00008200 \\ \mathrm{O} & 4.43973000 & -1.11718900 & 0.00006700 \\ \mathrm{H} & 5.39756100 & -0.98726300 & 0.00013500 \\ \mathrm{H} & 2.22916700 & -2.08084300 & -0.00012100 \\ \mathrm{C} & -1.90066900 & 0.01266600 & -0.00002600 \\ \mathrm{H} & -0.23718800 & -2.06693900 & -0.00021400 \\ \mathrm{H} & -0.21111000 & 2.21380700 & -0.00016300 \\ \mathrm{Cl} & -2.67490200 & 1.60831700 & -0.00105100 \\ \mathrm{Cl} & -2.46267300 & -0.87200600 & -1.44804700 \\ \mathrm{Cl} & -2.46214300 & -0.86996500 & 1.44946100\end{array}$

$\begin{array}{lrrr}\text { 4-COMe } & & & \\ \text { C } & 0.75387100 & -1.19713600 & -0.00010900 \\ \text { C } & 1.39803500 & 0.03767500 & 0.00005600 \\ \text { C } & 0.66028200 & 1.21495300 & 0.00016400 \\ \text { C } & -0.72312400 & 1.15915000 & 0.00010600 \\ \text { C } & -1.37448200 & -0.07194600 & -0.00005800 \\ \text { C } & -0.62781100 & -1.24769700 & -0.00016200 \\ \text { C } & 2.88194100 & 0.14936200 & 0.00012300 \\ \text { O } & 3.48709000 & 1.18679100 & 0.00026300 \\ \text { H } & 1.18540900 & 2.15963000 & 0.00029300 \\ \text { O } & 3.49953700 & -1.04561000 & 0.00001000 \\ \text { H } & 4.45023800 & -0.87123500 & 0.00006400 \\ \text { H } & 1.33804100 & -2.10549500 & -0.00019100 \\ \text { H } & -1.15363100 & -2.19196200 & -0.00028500 \\ \text { H } & -1.29081500 & 2.07893000 & 0.00019200 \\ \text { C } & -2.87314700 & -0.18115500 & -0.00012000\end{array}$




$\begin{array}{lrrr}\text { O } & -3.40427000 & -1.26515900 & -0.00020300 \\ \mathrm{C} & -3.68588100 & 1.08881600 & -0.00007700 \\ \mathrm{H} & -3.45333100 & 1.68978600 & 0.87979700 \\ \mathrm{H} & -3.45308200 & 1.69002800 & -0.87971900 \\ \mathrm{H} & -4.73979800 & 0.83000300 & -0.00025300\end{array}$

$\begin{array}{lrrr}\text { 4-CF } & & & \\ \text { C } & -1.01934400 & -1.17122900 & 0.00003600 \\ \text { C } & -1.70568300 & 0.03997600 & 0.00000800 \\ \text { C } & -1.01221400 & 1.24296100 & -0.00004600 \\ \text { C } & 0.37336700 & 1.24293100 & -0.00008100 \\ \text { C } & 1.05146300 & 0.03417300 & -0.00005300 \\ \text { C } & 0.36359800 & -1.17285800 & 0.00001500 \\ \text { C } & -3.19348700 & 0.09687500 & 0.00000800 \\ \text { O } & -3.83476100 & 1.11153000 & -0.00003500 \\ \text { H } & -1.57173800 & 2.16757300 & -0.00006800 \\ \text { O } & -3.76480100 & -1.12051700 & 0.00005700 \\ \text { H } & -4.72177400 & -0.98394300 & 0.00004900 \\ \text { H } & -1.56941600 & -2.10032100 & 0.00004400 \\ H & 0.91183300 & -2.10523500 & -0.00002900 \\ \text { H } & 0.92323900 & 2.17238600 & -0.00013500 \\ \text { C } & 2.55436100 & -0.00807700 & -0.00001200 \\ \text { F } & 3.02531600 & -0.65730200 & -1.07343800 \\ \text { F } & 3.02540400 & -0.65395300 & 1.07539900 \\ \text { F } & 3.09949900 & 1.21046800 & -0.00188200\end{array}$

$\begin{array}{lrrr}4-C\left(\mathrm{CF}_{3}\right)_{3} \mathbf{r}_{3} & & & \\ \mathrm{C} & -2.48550300 & -1.13820200 & 0.03709800 \\ \mathrm{C} & -3.17921800 & 0.06385200 & 0.03814100 \\ \mathrm{C} & -2.47701800 & 1.25872700 & 0.03241100 \\ \mathrm{C} & -1.09262900 & 1.25885400 & 0.04011500 \\ \mathrm{C} & -0.38367600 & 0.05787100 & 0.06638500 \\ \mathrm{C} & -1.10199100 & -1.14003800 & 0.04554900 \\ \mathrm{C} & -4.66629800 & 0.12121500 & 0.03110900 \\ \mathrm{O} & -5.30629100 & 1.13647000 & 0.02601800 \\ \mathrm{H} & -3.02583400 & 2.18968900 & 0.01702500 \\ \mathrm{O} & -5.23652000 & -1.09633700 & 0.03100300 \\ \mathrm{H} & -6.19374900 & -0.96139600 & 0.02584900 \\ \mathrm{H} & -3.02525200 & -2.07340500 & 0.02460500 \\ \mathrm{H} & -0.59156300 & -2.08828200 & 0.02664700 \\ \mathrm{H} & -0.59205300 & 2.21114100 & 0.01822900 \\ \mathrm{C} & 1.15291400 & 0.00675900 & 0.01992600 \\ \mathrm{C} & 1.75117200 & -0.77004300 & 1.23219600 \\ \mathrm{C} & 1.57942300 & -0.70918300 & -1.29914500 \\ \mathrm{C} & 1.80161800 & 1.41980900 & 0.02009400 \\ \mathrm{~F} & 1.53596400 & 2.04512900 & -1.12765100 \\ \mathrm{~F} & 3.12145200 & 1.36027200 & 0.15544700 \\ \mathrm{~F} & 1.34913700 & 2.18068200 & 1.01398000 \\ \mathrm{~F} & 0.81894200 & -0.31467400 & -2.31390700\end{array}$




$\begin{array}{llrr}F & 1.44245500 & -2.03156600 & -1.17722600 \\ F & 2.84331000 & -0.46777400 & -1.62678500 \\ F & 3.01997800 & -1.10489300 & 1.01018800 \\ F & 1.09322800 & -1.89295700 & 1.49990700 \\ F & 1.70644300 & -0.01605700 & 2.32695900\end{array}$

$\begin{array}{lrrr}\text { 4-CN } & & & \\ \mathrm{C} & 0.27967300 & -1.18468100 & 0.00000700 \\ \mathrm{C} & 0.95213700 & 0.03260000 & 0.00000500 \\ \mathrm{C} & 0.24583900 & 1.23074000 & -0.00000500 \\ \mathrm{C} & -1.13579300 & 1.21749500 & -0.00001300 \\ \mathrm{C} & -1.80882100 & -0.00392000 & -0.00001100 \\ \mathrm{C} & -1.10378300 & -1.20558300 & 0.00000000 \\ \mathrm{C} & 2.43978000 & 0.10571000 & 0.00001300 \\ \mathrm{O} & 3.06754500 & 1.12844300 & 0.00001200 \\ \mathrm{H} & 0.79646500 & 2.16067600 & -0.00000600 \\ \mathrm{O} & 3.02341200 & -1.10500600 & 0.00002200 \\ \mathrm{H} & 3.97924300 & -0.96000500 & 0.00002700 \\ \mathrm{H} & 0.83985600 & -2.10774400 & 0.00001500 \\ \mathrm{H} & -1.64246600 & -2.14194600 & 0.00000100 \\ \mathrm{H} & -1.69927900 & 2.13912500 & -0.00002000 \\ \mathrm{C} & -3.24493500 & -0.02417700 & -0.00001800 \\ \mathrm{~N} & -4.39229300 & -0.04095900 & -0.00002500\end{array}$

\section{4-SO ${ }_{2} \mathrm{Ph}$}

C

C $\quad-2.90039100 \quad-0.18602400 \quad 0.03730400$

C $\quad-2.24599000 \quad 0.06838400 \quad 1.23851000$

C $\quad-0.95763000 \quad 0.57331200 \quad 1.22780400$

C $\quad-0.34590100 \quad 0.81275100 \quad 0.00606100$

C $\quad-0.98690700 \quad 0.57296300 \quad-1.19881100$

C $\quad-4.28593800 \quad-0.72828300 \quad 0.10681700$

$\begin{array}{llll}\mathrm{O} & -4.87080900 & -0.96800800 & 1.12716300\end{array}$

$\begin{array}{llll}\mathrm{H} & -2.76372700 & -0.12892400 & 2.16645900\end{array}$

O $\quad-4.83216300 \quad-0.92964200-1.10521800$

$\mathrm{H} \quad-5.72268000 \quad-1.27661200-0.96011800$

$\mathrm{H} \quad-2.80449800 \quad-0.12257000 \quad-2.10317800$

$\mathrm{H} \quad-0.48418000 \quad 0.80033300 \quad-2.12840800$

$\mathrm{H} \quad-0.43297400 \quad 0.80150100 \quad 2.14501900$

S $\quad \begin{array}{llll}1.32298100 & 1.44944400 & -0.01509200\end{array}$

$\begin{array}{lllll}\mathrm{O} & 1.51625400 & 2.11525700 & -1.28834900\end{array}$

$\begin{array}{llll}O & 1.53815400 & 2.14202600 & 1.24007700\end{array}$

C $\quad 2.32936600 \quad-0.01822900-0.00806700$

C $\quad 2.71792100 \quad-0.56039400 \quad 1.20734300$

C $\quad 2.69576100 \quad-0.58730900-1.21807000$

C $\quad 3.48979700 \quad-1.71236800 \quad 1.20629000$

H $\quad 2.43411600 \quad-0.07035900 \quad 2.12847000$

C $\quad 3.46783400 \quad-1.73903700 \quad-1.20558700$

H $\quad 2.39511000 \quad-0.11763600 \quad-2.14444000$ 


$\begin{array}{llrr}\mathrm{C} & 3.85928500 & -2.30005700 & 0.00325200 \\ \mathrm{H} & 3.80856400 & -2.14692400 & 2.14326400 \\ \mathrm{H} & 3.76974200 & -2.19429000 & -2.13832500 \\ \mathrm{H} & 4.46314600 & -3.19712000 & 0.00770100\end{array}$

$\begin{array}{lrrr}\text { 4-SO } & \\ \mathrm{Me} & & & \\ \mathrm{C} & 1.31241000 & 1.18264200 & -0.03723400 \\ \mathrm{C} & 1.98495900 & -0.03470700 & -0.01166300 \\ \mathrm{C} & 1.28488100 & -1.23665900 & -0.04292700 \\ \mathrm{C} & -0.09833700 & -1.22705700 & -0.09253900 \\ \mathrm{C} & -0.75571700 & -0.00536400 & -0.10554100 \\ \mathrm{C} & -0.07288000 & 1.20037500 & -0.08523000 \\ \mathrm{C} & 3.47276400 & -0.10374100 & 0.04044200 \\ \mathrm{O} & 4.10319200 & -1.12442700 & 0.06870300 \\ \mathrm{H} & 1.84004300 & -2.16393800 & -0.03532300 \\ \mathrm{O} & 4.05352800 & 1.10832900 & 0.05478100 \\ \mathrm{H} & 5.00905600 & 0.96496500 & 0.08666700 \\ \mathrm{H} & 1.87231400 & 2.10589300 & -0.02639000 \\ \mathrm{H} & -0.62444200 & 2.12934500 & -0.12875700 \\ \mathrm{H} & -0.66897800 & -2.14414700 & -0.14278000 \\ \mathrm{~S} & -2.54062100 & 0.01463700 & -0.14731100 \\ \mathrm{O} & -2.95995100 & 1.29001000 & -0.69455900 \\ \mathrm{O} & -2.98724500 & -1.22934300 & -0.74261200 \\ \mathrm{C} & -2.99174000 & -0.01342500 & 1.57545800 \\ \mathrm{H} & -2.60598200 & -0.92626800 & 2.02075200 \\ \mathrm{H} & -2.58666900 & 0.87328200 & 2.05504700 \\ \mathrm{H} & -4.07962000 & -0.00225000 & 1.59265300\end{array}$

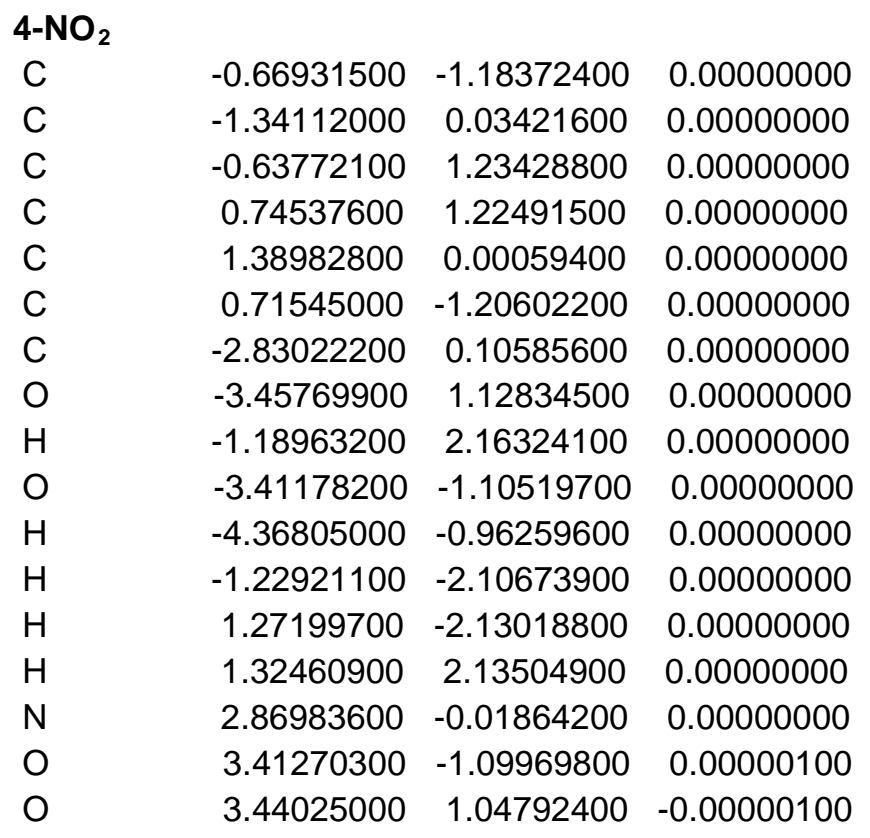

4- $\mathrm{COCF}_{3}$ 


$\begin{array}{lrrr}\text { C } & -1.81189400 & 1.29091700 & 0.00009000 \\ \text { C } & -2.28130800 & -0.01988100 & -0.00000500 \\ \text { C } & -1.39311900 & -1.08795200 & -0.00012300 \\ \text { C } & -0.02919100 & -0.85346400 & -0.00014100 \\ \text { C } & 0.44514500 & 0.45777300 & -0.00005200 \\ \text { C } & -0.45065300 & 1.52631900 & 0.00006200 \\ \text { C } & -3.73922300 & -0.33100600 & 0.00000900 \\ \text { O } & -4.19462400 & -1.44111800 & -0.00008000 \\ \text { H } & -1.78712900 & -2.09420500 & -0.00020100 \\ \text { O } & -4.50902400 & 0.77036400 & 0.00012900 \\ \text { H } & -5.42932900 & 0.47420700 & 0.00012700 \\ \text { H } & -2.51246400 & 2.11255500 & 0.00018300 \\ \text { H } & -0.05859600 & 2.53346800 & 0.00012300 \\ \text { H } & 0.65113600 & -1.69178300 & -0.00024300 \\ \text { C } & 1.89353500 & 0.79642100 & -0.00010500 \\ \text { O } & 2.31788800 & 1.91651000 & -0.00030200 \\ \text { C } & 2.91653800 & -0.36848200 & 0.00006900 \\ \text { F } & 4.15475800 & 0.08414000 & 0.00011300 \\ \text { F } & 2.75163300 & -1.13998700 & 1.08099700 \\ \text { F } & 2.75177300 & -1.14017000 & -1.08075300\end{array}$

$\begin{array}{lrrr}4-\mathrm{SO}_{2} \mathrm{~F} & & & \\ \mathrm{C} & -1.29291400 & -1.18371800 & -0.03408600 \\ \mathrm{C} & -1.96412400 & 0.03411300 & -0.01515300 \\ \mathrm{C} & -1.26562300 & 1.23695700 & -0.03269900 \\ \mathrm{C} & 0.11732300 & 1.23040500 & -0.06717100 \\ \mathrm{C} & 0.76848600 & 0.00554800 & -0.07850200 \\ \mathrm{C} & 0.09203200 & -1.20369500 & -0.06747600 \\ \mathrm{C} & -3.45453600 & 0.10337200 & 0.01795500 \\ \mathrm{O} & -4.08135800 & 1.12561700 & 0.03421700 \\ \mathrm{H} & -1.82106400 & 2.16385400 & -0.02414700 \\ \mathrm{O} & -4.03272100 & -1.10835100 & 0.02825300 \\ \mathrm{H} & -4.98941700 & -0.96956900 & 0.04967700 \\ \mathrm{H} & -1.85264100 & -2.10686700 & -0.02746600 \\ \mathrm{H} & 0.64350100 & -2.13248100 & -0.09563800 \\ \mathrm{H} & 0.68788400 & 2.14763800 & -0.09570700 \\ \mathrm{~S} & 2.53247000 & -0.01440500 & -0.12026200 \\ \mathrm{O} & 3.00730700 & -1.28113400 & -0.57704000 \\ \mathrm{O} & 3.03510300 & 1.22193900 & -0.62723900 \\ \mathrm{~F} & 2.82018900 & 0.01393800 & 1.43497000\end{array}$

\section{4- $\mathrm{SO}_{2} \mathrm{CF}_{3}$}

$\begin{array}{lrrr}C & -1.92154000 & -1.17605600 & -0.20917600 \\ C & -2.58368500 & 0.03589000 & -0.04225900 \\ C & -1.90009700 & 1.24485800 & -0.12406600 \\ C & -0.53885600 & 1.25029000 & -0.37132300 \\ C & 0.10565800 & 0.03140000 & -0.53132600 \\ C & -0.55856100 & -1.18365000 & -0.45710700 \\ C & -4.05105000 & 0.09182000 & 0.22675000\end{array}$




$\begin{array}{lrrr}\mathrm{O} & -4.66954900 & 1.10771500 & 0.37972300 \\ \mathrm{H} & -2.45042200 & 2.16594100 & 0.00446600 \\ \mathrm{O} & -4.61765400 & -1.12402500 & 0.28215200 \\ \mathrm{H} & -5.55940900 & -0.99395700 & 0.45872800 \\ \mathrm{H} & -2.47201900 & -2.10279800 & -0.14833100 \\ \mathrm{H} & -0.01440800 & -2.10599500 & -0.60352700 \\ \mathrm{H} & 0.02021000 & 2.17171800 & -0.45312100 \\ \mathrm{~S} & 1.84965800 & 0.02613300 & -0.84066300 \\ \mathrm{O} & 2.23177100 & -1.22082800 & -1.45471300 \\ \mathrm{O} & 2.25615900 & 1.30625300 & -1.36369800 \\ \mathrm{C} & 2.53220100 & -0.04315800 & 0.89122000 \\ \mathrm{~F} & 2.10834900 & -1.14105900 & 1.49935000 \\ \mathrm{~F} & 3.84889100 & -0.05326500 & 0.82810000 \\ \mathrm{~F} & 2.12845900 & 1.01495300 & 1.57792700\end{array}$

\begin{tabular}{|c|c|c|c|}
\hline \multicolumn{4}{|c|}{$4-\mathrm{SO}_{2} \mathrm{Cl}$} \\
\hline $\mathrm{C}$ & 1.52565400 & 1.18300700 & -0.09608800 \\
\hline C & 2.19482300 & -0.03441600 & -0.02575700 \\
\hline C & 1.49992400 & -1.23808300 & -0.08769400 \\
\hline C & 0.12242600 & -1.23355400 & -0.21700600 \\
\hline C & -0.52331100 & -0.00859300 & -0.28164700 \\
\hline C & 0.14632500 & 1.20227600 & -0.22554800 \\
\hline C & 3.67875500 & -0.10219000 & 0.11335100 \\
\hline $\mathrm{O}$ & 4.30422100 & -1.12364200 & 0.17520500 \\
\hline $\mathrm{H}$ & 2.05397000 & -2.16449000 & -0.03633100 \\
\hline $\mathrm{O}$ & 4.25397600 & 1.11014000 & 0.16375200 \\
\hline $\mathrm{H}$ & 5.20675900 & 0.97162500 & 0.25298400 \\
\hline $\mathrm{H}$ & 2.08297500 & 2.10667700 & -0.05278800 \\
\hline $\mathrm{H}$ & -0.40366800 & 2.13003700 & -0.29264500 \\
\hline $\mathrm{H}$ & -0.44574000 & -2.15075900 & -0.27771100 \\
\hline $\mathrm{S}$ & -2.28855400 & 0.00983500 & -0.45463600 \\
\hline $\mathrm{O}$ & -2.70684400 & 1.26917300 & -0.99983500 \\
\hline $\mathrm{O}$ & -2.73282600 & -1.23811500 & -1.00507800 \\
\hline $\mathrm{Cl}$ & -2.86430700 & 0.01167200 & 1.52533200 \\
\hline
\end{tabular}

B. Cartesian Coordinates of geometry optimized meta-substituted benzoic acids

$\begin{array}{lrrr}\text { 3-NMe }{ }_{2} & & & \\ \mathrm{C} & 0.08160300 & -0.48074900 & -0.05327700 \\ \mathrm{C} & 1.26735500 & 0.23924800 & -0.01073500 \\ \mathrm{C} & 1.27111600 & 1.62818300 & 0.01820900 \\ \mathrm{C} & 0.05427800 & 2.28739300 & 0.00954100 \\ \mathrm{C} & -1.14004000 & 1.58643600 & -0.03266100 \\ \mathrm{C} & -1.15496000 & 0.18192600 & -0.07924000 \\ \mathrm{C} & 2.58103300 & -0.45596200 & 0.01177900 \\ \mathrm{O} & 3.64891800 & 0.09433600 & 0.04692700 \\ \mathrm{H} & 2.21140900 & 2.15755500 & 0.05355700 \\ \mathrm{O} & 2.47687500 & -1.80108600 & -0.01142300\end{array}$




$\begin{array}{lrrr}\mathrm{H} & 3.37931600 & -2.14570500 & 0.00571100 \\ \mathrm{H} & 0.13350300 & -1.55680800 & -0.06693500 \\ \mathrm{H} & 0.02618600 & 3.36809300 & 0.04084000 \\ \mathrm{H} & -2.06635700 & 2.13981700 & -0.03070100 \\ \mathrm{~N} & -2.34059200 & -0.52230000 & -0.15360300 \\ \mathrm{C} & -3.58207700 & 0.18014500 & 0.08686500 \\ \mathrm{H} & -4.40610000 & -0.52402400 & 0.01770300 \\ \mathrm{H} & -3.61061600 & 0.65386800 & 1.07541400 \\ \mathrm{H} & -3.74425100 & 0.95274300 & -0.66581000 \\ \mathrm{C} & -2.31234300 & -1.95364900 & 0.05735200 \\ \mathrm{H} & -3.32411800 & -2.34177900 & -0.01586200 \\ \mathrm{H} & -1.71258400 & -2.44721800 & -0.70810000 \\ \mathrm{H} & -1.90438100 & -2.22427400 & 1.03837800\end{array}$

$\begin{array}{lrrr}\text { 3-NEt } & & & \\ \mathrm{C} & & & \\ \mathrm{C} & 0.56669800 & 0.33337500 & -0.22168000 \\ \mathrm{C} & 1.77578900 & -0.31665600 & -0.01918700 \\ \mathrm{C} & 1.84491200 & -1.69715300 & 0.11782200 \\ \mathrm{C} & 0.66460700 & -2.41649000 & 0.05269300 \\ \mathrm{C} & -0.55205600 & -1.78553900 & -0.14786600 \\ \mathrm{C} & -0.63525300 & -0.38957100 & -0.30703200 \\ \mathrm{O} & 3.04601500 & 0.45077300 & 0.06507200 \\ \mathrm{H} & 4.13316100 & -0.03509800 & 0.22795600 \\ \mathrm{O} & 2.80133200 & -2.17144500 & 0.27800900 \\ \mathrm{H} & 2.87787600 & 1.78435100 & -0.05939300 \\ \mathrm{H} & 3.75756400 & 2.17790100 & 0.00884000 \\ \mathrm{H} & 0.57641700 & 1.40673600 & -0.31108900 \\ \mathrm{H} & 0.68173300 & -3.49221400 & 0.16418900 \\ \mathrm{~N} & -1.44373600 & -2.39203600 & -0.17977700 \\ \mathrm{C} & -1.83685800 & 0.24077600 & -0.55743400 \\ \mathrm{H} & -3.07998600 & -0.50764600 & -0.52740900 \\ \mathrm{H} & -3.83121600 & 0.09309100 & -1.03900300 \\ \mathrm{C} & -2.96481600 & -1.41481200 & -1.12322800 \\ \mathrm{H} & -1.90945000 & 1.68987300 & -0.61250800 \\ \mathrm{H} & -2.84119800 & 1.94734800 & -1.11514800 \\ \mathrm{C} & -1.10886300 & 2.06239000 & -1.25371200 \\ \mathrm{H} & -3.57249500 & -0.85806100 & 0.87547600 \\ \mathrm{H} & -4.46482800 & -1.48221100 & 0.82204400 \\ \mathrm{H} & -2.80834900 & -1.39785100 & 1.43368600 \\ \mathrm{C} & -3.82087500 & 0.04340300 & 1.43361300 \\ \mathrm{H} & -1.85486700 & 2.37760900 & 0.75019200 \\ \mathrm{H} & -1.84340400 & 3.46104500 & 0.62962500 \\ \mathrm{H} & -2.72245900 & 2.11140700 & 1.35220000 \\ & -0.96107600 & 2.08471500 & 1.29984400\end{array}$
3- $\mathrm{NH}_{2}$
C
$\begin{array}{lll}-0.40172200 & -0.81919000 & -0.00883300\end{array}$
$\begin{array}{llll}\text { C } & 0.56658300 & 0.17559400 & -0.00153100\end{array}$
$\begin{array}{lllll}\text { C } & 0.21313600 & 1.51997400 & 0.00467400\end{array}$ 


$\begin{array}{lrrr}\mathrm{C} & -1.13078500 & 1.85550300 & 0.00367000 \\ \mathrm{C} & -2.10526800 & 0.87064200 & -0.00363800 \\ \mathrm{C} & -1.75336700 & -0.48014400 & -0.00847400 \\ \mathrm{C} & 2.01419500 & -0.16128500 & 0.00136900 \\ \mathrm{O} & 2.90652000 & 0.64333600 & 0.00495400 \\ \mathrm{H} & 0.98938400 & 2.27054800 & 0.01078400 \\ \mathrm{O} & 2.25221900 & -1.48874900 & 0.00015000 \\ \mathrm{H} & 3.21244300 & -1.59640100 & 0.00018900 \\ \mathrm{H} & -0.10470900 & -1.85879300 & -0.01823900 \\ \mathrm{H} & -1.42776700 & 2.89517700 & 0.00885000 \\ \mathrm{H} & -3.15304200 & 1.14422600 & -0.00980700 \\ \mathrm{~N} & -2.73107700 & -1.46844300 & -0.07025700 \\ \mathrm{H} & -2.44753000 & -2.37706000 & 0.25852800 \\ \mathrm{H} & -3.63778100 & -1.20186300 & 0.27724500\end{array}$

$\begin{array}{lrrr}\text { 3-O-iPr } & & & \\ \text { C } & -0.62700100 & -0.66978100 & -0.00001900 \\ \text { C } & -1.72688000 & 0.16996000 & -0.00000400 \\ \text { C } & -1.56832300 & 1.55404700 & 0.00001100 \\ \text { C } & -0.29009500 & 2.07396400 & 0.00000400 \\ \text { C } & 0.82687200 & 1.24249800 & -0.00002100 \\ \text { C } & 0.66463800 & -0.14034600 & -0.00002900 \\ \text { C } & -3.11098300 & -0.37234100 & 0.00000300 \\ \text { O } & -4.10830000 & 0.29816100 & 0.00001900 \\ \text { H } & -2.44325500 & 2.18704000 & 0.00002600 \\ \text { O } & -3.15528300 & -1.71869000 & -0.00001000 \\ \text { H } & -4.08956300 & -1.96512100 & -0.00001400 \\ \text { H } & -0.74682000 & -1.74296200 & -0.00003000 \\ \text { H } & -0.14316400 & 3.14525700 & 0.00001700 \\ \text { O } & 1.64989100 & -1.06973700 & -0.00005700 \\ \text { C } & 3.04668200 & -0.75696500 & 0.00000500 \\ \text { C } & 3.49894100 & -0.06750700 & 1.27933200 \\ \text { H } & 3.19646800 & 0.97529900 & 1.33581700 \\ \text { H } & 3.09764200 & -0.59058100 & 2.14604800 \\ \text { H } & 4.58719200 & -0.10130400 & 1.33196800 \\ \text { H } & 1.80610000 & 1.68891000 & -0.00005400 \\ \text { C } & 3.49903400 & -0.06744400 & -1.27925600 \\ \text { H } & 3.09776700 & -0.59045400 & -2.14602500 \\ H & 3.19658700 & 0.97537100 & -1.33569100 \\ \text { H } & 4.58728700 & -0.10126100 & -1.33183600 \\ \text { H } & 3.48598600 & -1.75458100 & 0.00000000\end{array}$

\section{3-OBn}

$\begin{array}{lrrr}\text { C } & 1.76569900 & -0.77105600 & 0.06212400 \\ \text { C } & 2.70280500 & 0.24866400 & 0.05310600 \\ \text { C } & 2.38733400 & 1.51189000 & 0.54847000 \\ \text { C } & 1.11984100 & 1.73748500 & 1.04786800 \\ \text { C } & 0.16449200 & 0.72550200 & 1.06223100 \\ \text { C } & 0.49228200 & -0.53412000 & 0.57199300\end{array}$




$\begin{array}{lrrr}\mathrm{C} & 4.07308900 & 0.03218100 & -0.48092300 \\ \mathrm{O} & 4.93402600 & 0.86964600 & -0.51533100 \\ \mathrm{H} & 3.13829900 & 2.28784000 & 0.52828400 \\ \mathrm{O} & 4.27379700 & -1.21933400 & -0.93697200 \\ \mathrm{H} & 5.18438600 & -1.25498500 & -1.25828300 \\ \mathrm{H} & 2.00523900 & -1.75375900 & -0.31625500 \\ \mathrm{H} & 0.85533400 & 2.71388700 & 1.42928100 \\ \mathrm{H} & -0.82611500 & 0.93541500 & 1.43694300 \\ \mathrm{O} & -0.35524600 & -1.59815200 & 0.54874600 \\ \mathrm{C} & -1.64388000 & -1.44648500 & 1.10683100 \\ \mathrm{H} & -2.03022800 & -2.46221300 & 1.18833900 \\ \mathrm{H} & -1.57299900 & -1.03686100 & 2.11896400 \\ \mathrm{C} & -2.57902700 & -0.60904000 & 0.26683400 \\ \mathrm{C} & -3.70194400 & -0.03788600 & 0.85549900 \\ \mathrm{C} & -2.35266400 & -0.42245500 & -1.09072900 \\ \mathrm{C} & -4.59481000 & 0.70335300 & 0.09591800 \\ \mathrm{H} & -3.87729100 & -0.17160600 & 1.91671600 \\ \mathrm{C} & -3.24214600 & 0.32484600 & -1.85029800 \\ \mathrm{H} & -1.47408000 & -0.85737300 & -1.54805700 \\ \mathrm{C} & -4.36550600 & 0.88703400 & -1.26088300 \\ \mathrm{H} & -5.46483600 & 1.14327800 & 0.56400500 \\ \mathrm{H} & -3.05526100 & 0.46974300 & -2.90559000 \\ \mathrm{H} & -5.05645300 & 1.46988300 & -1.85413900\end{array}$

$\begin{array}{lrrr}\text { 3-OH } & & & \\ \mathrm{C} & 0.39117100 & -0.84504400 & -0.00000300 \\ \mathrm{C} & -0.55724200 & 0.16783900 & -0.00000200 \\ \mathrm{C} & -0.17731200 & 1.50643700 & 0.00000900 \\ \mathrm{C} & 1.16908400 & 1.82421900 & 0.00001700 \\ \mathrm{C} & 2.12874900 & 0.82086100 & 0.00001500 \\ \mathrm{C} & 1.73902300 & -0.51267000 & 0.00000500 \\ \mathrm{C} & -2.01250800 & -0.14013600 & -0.00001100 \\ \mathrm{O} & -2.88554300 & 0.68511900 & -0.00001000 \\ \mathrm{H} & -0.94136900 & 2.26965800 & 0.00001000 \\ \mathrm{O} & -2.27430200 & -1.46077900 & -0.00002000 \\ \mathrm{H} & -3.23610600 & -1.55433800 & -0.00002600 \\ \mathrm{H} & 0.09989000 & -1.88470500 & -0.00001100 \\ \mathrm{H} & 1.48208700 & 2.85889700 & 0.00002500 \\ \mathrm{H} & 3.18268100 & 1.07274400 & 0.00002200 \\ \mathrm{O} & 2.63435400 & -1.53682700 & 0.00000300 \\ \mathrm{H} & 3.53095700 & -1.19139500 & 0.00000900\end{array}$

$\begin{array}{lrrr}\text { 3-OMe } & & & \\ \text { C } & -0.06746300 & -0.70787700 & 0.00020400 \\ \text { C } & 1.00403700 & 0.16787900 & 0.00006400 \\ \text { C } & 0.80094100 & 1.54713600 & -0.00000100 \\ \text { C } & -0.49090000 & 2.03256500 & 0.00005200 \\ \text { C } & -1.58137300 & 1.16620200 & 0.00015500 \\ \text { C } & -1.36808400 & -0.20794700 & 0.00024700\end{array}$




$\begin{array}{lrrr}\mathrm{C} & 2.40565400 & -0.32859600 & -0.00007100 \\ \mathrm{O} & 3.37977700 & 0.37475100 & -0.00023500 \\ \mathrm{H} & 1.65728000 & 2.20519200 & -0.00009600 \\ \mathrm{O} & 2.49293100 & -1.67245900 & -0.00001500 \\ \mathrm{H} & 3.43442600 & -1.88994700 & -0.00015300 \\ \mathrm{H} & 0.08346900 & -1.77713700 & 0.00025600 \\ \mathrm{H} & -0.66722400 & 3.09927400 & 0.00000600 \\ \mathrm{H} & -2.58171400 & 1.57183900 & 0.00018700 \\ \mathrm{O} & -2.35724400 & -1.13503300 & 0.00044000 \\ \mathrm{C} & -3.69063600 & -0.67415900 & -0.00054100 \\ \mathrm{H} & -4.31807500 & -1.55999000 & -0.00025500 \\ \mathrm{H} & -3.90295100 & -0.07866100 & 0.89039500 \\ \mathrm{H} & -3.90198100 & -0.07985900 & -0.89250800\end{array}$

$\begin{array}{lrrr}\text { 3-OSiMe } & & & \\ \mathrm{C} & & & \\ \mathrm{C} & 0.89100300 & -0.29500900 & -0.46232100 \\ \mathrm{C} & 2.15244800 & 0.15356400 & -0.08836900 \\ \mathrm{C} & 2.38338300 & 1.50210300 & 0.15977200 \\ \mathrm{C} & 1.33995200 & 2.40363800 & 0.03087500 \\ \mathrm{C} & 0.07651200 & 1.96626000 & -0.34074300 \\ \mathrm{C} & -0.15064900 & 0.61565500 & -0.58726000 \\ \mathrm{O} & 3.29459300 & -0.78603200 & 0.05679700 \\ \mathrm{H} & 4.40974800 & -0.46672700 & 0.37021300 \\ \mathrm{O} & 3.37533500 & 1.81956700 & 0.44663300 \\ \mathrm{H} & 2.96299300 & -2.06683100 & -0.19917300 \\ \mathrm{H} & 3.76662900 & -2.59019100 & -0.08083400 \\ \mathrm{H} & 1.50786900 & 3.45512200 & 0.21810300 \\ \mathrm{H} & 0.71112800 & -1.34110000 & -0.66364000 \\ \mathrm{O} & -0.74668600 & 2.65945100 & -0.45119700 \\ \mathrm{Si} & -1.38351300 & 0.19260400 & -0.97711500 \\ \mathrm{C} & -2.56017200 & -0.33195200 & 0.10304300 \\ \mathrm{H} & -1.90465400 & -1.80511500 & 1.04552900 \\ \mathrm{H} & -1.65007800 & -2.62278600 & 0.37023700 \\ \mathrm{H} & -1.01027600 & -1.54154500 & 1.61217000 \\ \mathrm{C} & -2.65158100 & -2.17069800 & 1.75199400 \\ \mathrm{H} & -4.01614200 & -0.77570200 & -0.96632900 \\ \mathrm{H} & -4.84946100 & -1.13257500 & -0.35995100 \\ \mathrm{H} & -4.35534700 & 0.09065900 & -1.53432800 \\ \mathrm{C} & -3.74926600 & -1.56143000 & -1.67322300 \\ \mathrm{H} & -2.95232300 & 1.06323500 & 1.28200000 \\ \mathrm{H} & -2.06502700 & 1.36034200 & 1.84337600 \\ \mathrm{H} & -3.32500300 & 1.93722200 & 0.74649000 \\ & -3.71437700 & 0.75735000 & 2.00046700\end{array}$

$\begin{array}{lrrr}\text { 3-OEt } & & & \\ \text { C } & -0.27462900 & -0.57026600 & -0.00002700 \\ \text { C } & -1.47074000 & 0.12580700 & -0.00000500 \\ \text { C } & -1.48738000 & 1.51976100 & 0.00000600 \\ \text { C } & -0.28777400 & 2.20201400 & -0.00000500\end{array}$




$\begin{array}{lrrr}\text { C } & 0.92536600 & 1.51823500 & -0.00002400 \\ \text { C } & 0.93195300 & 0.12703500 & -0.00003600 \\ \text { C } & -2.77650500 & -0.58514400 & 0.00001300 \\ \text { O } & -3.84949200 & -0.04430900 & 0.00005500 \\ H & -2.43655500 & 2.03490700 & 0.00002300 \\ \text { O } & -2.65134400 & -1.92619800 & -0.00002300 \\ H & -3.54704200 & -2.28869500 & -0.00000400 \\ H & -0.25605100 & -1.64999200 & -0.00003500 \\ H & -0.28132900 & 3.28322600 & 0.00000200 \\ \text { O } & 2.05393000 & -0.63265400 & -0.00005500 \\ \text { C } & 3.31003400 & 0.02558400 & 0.00002700 \\ \text { C } & 4.38232000 & -1.03768500 & 0.00004600 \\ H & 3.38962300 & 0.66246200 & 0.88616600 \\ H & 3.38971400 & 0.66252200 & -0.88606000 \\ H & 4.29203600 & -1.66684200 & 0.88378700 \\ H & 4.29214100 & -1.66677100 & -0.88375700 \\ H & 5.36785900 & -0.57425600 & 0.00012300 \\ H & 1.84898300 & 2.07668000 & -0.00003200\end{array}$

$\begin{array}{lrrr}\text { 3-Cyclopropyl } & & \\ \mathrm{C} & 0.17767300 & -0.62905900 & 0.00001600 \\ \mathrm{C} & 1.32201900 & 0.16115600 & -0.00000200 \\ \mathrm{C} & 1.22244700 & 1.54695300 & -0.00003500 \\ \mathrm{C} & -0.03245500 & 2.13294500 & -0.00005100 \\ \mathrm{C} & -1.17261500 & 1.34321100 & -0.00003400 \\ \mathrm{C} & -1.08661300 & -0.04918800 & 0.00000000 \\ \mathrm{C} & 2.68131400 & -0.43794500 & 0.00001400 \\ \mathrm{O} & 3.70723700 & 0.18810200 & 0.00000100 \\ \mathrm{H} & 2.12634000 & 2.13909700 & -0.00004800 \\ \mathrm{O} & 2.66818200 & -1.78580800 & 0.00004600 \\ \mathrm{H} & 3.59137000 & -2.07110600 & 0.00005400 \\ \mathrm{H} & 0.27571200 & -1.70584100 & 0.00004200 \\ \mathrm{H} & -0.12584100 & 3.21013100 & -0.00007700 \\ \mathrm{H} & -2.14410100 & 1.82248900 & -0.00004700 \\ \mathrm{C} & -2.29304700 & -0.91308300 & 0.00001900 \\ \mathrm{C} & -3.53252200 & -0.49599600 & 0.74593900 \\ \mathrm{C} & -3.53251800 & -0.49603200 & -0.74592600 \\ \mathrm{H} & -2.07692000 & -1.97213900 & 0.00004300 \\ \mathrm{H} & -3.49552600 & 0.45572200 & 1.25628500 \\ \mathrm{H} & -4.09048600 & -1.26504000 & 1.25818400 \\ \mathrm{H} & -4.09048400 & -1.26509800 & -1.25813600 \\ \mathrm{H} & -3.49552300 & 0.45566400 & -1.25631600 \\ & & & \end{array}$

$\begin{array}{lrrr}\text { 3-t-Bu } & & & \\ \text { C } & 0.29147900 & -0.39560000 & 0.00000000 \\ \text { C } & 1.52895800 & 0.23186800 & -0.00000100 \\ \text { C } & 1.61659700 & 1.62119600 & 0.00000300 \\ \text { C } & 0.45301200 & 2.36383900 & 0.00000900 \\ \text { C } & -0.78637100 & 1.72964900 & 0.00001000\end{array}$




$\begin{array}{lrrr}\mathrm{C} & -0.89303600 & 0.34191500 & 0.00000400 \\ \mathrm{C} & 2.79531100 & -0.54413300 & -0.00000300 \\ \mathrm{O} & 3.89573600 & -0.06081400 & -0.00002400 \\ \mathrm{H} & 2.59179000 & 2.08689700 & 0.00000300 \\ \mathrm{O} & 2.60309900 & -1.87864800 & 0.00002300 \\ \mathrm{H} & 3.48036800 & -2.28372500 & 0.00001800 \\ \mathrm{H} & 0.26087200 & -1.47602600 & -0.00000400 \\ \mathrm{H} & 0.49980700 & 3.44413900 & 0.00001400 \\ \mathrm{H} & -1.67706000 & 2.34098700 & 0.00001400 \\ \mathrm{C} & -2.23339600 & -0.39008800 & -0.00000200 \\ \mathrm{C} & -3.41724800 & 0.57624400 & 0.00001900 \\ \mathrm{H} & -4.34740500 & 0.00745200 & 0.00001700 \\ \mathrm{H} & -3.41590600 & 1.21312900 & 0.88561400 \\ \mathrm{H} & -3.41591900 & 1.21315400 & -0.88555700 \\ \mathrm{C} & -2.32895800 & -1.27258400 & -1.25228400 \\ \mathrm{H} & -1.53140100 & -2.01446800 & -1.28169300 \\ \mathrm{H} & -3.28278300 & -1.80260000 & -1.26267900 \\ \mathrm{H} & -2.26163900 & -0.66702800 & -2.15685100 \\ \mathrm{C} & -2.32895000 & -1.27262900 & 1.25224800 \\ \mathrm{H} & -2.26162200 & -0.66710700 & 2.15683700 \\ \mathrm{H} & -3.28277800 & -1.80264200 & 1.26263100 \\ \mathrm{H} & -1.53139700 & -2.01451800 & 1.28162400\end{array}$

$\begin{array}{lrrr}\text { 3-Me } & & & \\ \text { C } & -0.41466500 & -0.79271600 & 0.00000600 \\ \text { C } & 0.58468600 & 0.17149300 & 0.00000100 \\ \text { C } & 0.25930100 & 1.52493700 & -0.00001300 \\ \text { C } & -1.07114700 & 1.90022300 & -0.00002300 \\ \text { C } & -2.06821500 & 0.93117800 & -0.00001800 \\ \text { C } & -1.75694100 & -0.42409600 & -0.00000500 \\ \text { C } & 2.02370600 & -0.19672700 & 0.00001300 \\ \text { O } & 2.93285700 & 0.58930200 & 0.00000900 \\ \text { H } & 1.05611000 & 2.25499000 & -0.00001500 \\ \text { O } & 2.23174000 & -1.52844200 & 0.00002700 \\ \text { H } & 3.18920000 & -1.65876800 & 0.00003300 \\ H & -0.14325700 & -1.83995700 & 0.00001700 \\ H & -1.33849300 & 2.94782800 & -0.00003300 \\ H & -3.10791400 & 1.23407300 & -0.00002600 \\ \text { C } & -2.83193000 & -1.47557600 & -0.00000400 \\ \text { H } & -2.74911800 & -2.11552500 & -0.87884000 \\ H & -2.74908500 & -2.11555900 & 0.87880300 \\ H & -3.82298700 & -1.02626800 & 0.00002300\end{array}$

$\begin{array}{lrrr}\text { 3-i-Pr } & & & \\ \text { C } & 0.29147900 & -0.39560000 & 0.00000000 \\ \text { C } & 1.52895800 & 0.23186800 & -0.00000100 \\ \text { C } & 1.61659700 & 1.62119600 & 0.00000300 \\ \text { C } & 0.45301200 & 2.36383900 & 0.00000900 \\ \text { C } & -0.78637100 & 1.72964900 & 0.00001000\end{array}$




$\begin{array}{lrrr}\mathrm{C} & -0.89303600 & 0.34191500 & 0.00000400 \\ \mathrm{C} & 2.79531100 & -0.54413300 & -0.00000300 \\ \mathrm{O} & 3.89573600 & -0.06081400 & -0.00002400 \\ \mathrm{H} & 2.59179000 & 2.08689700 & 0.00000300 \\ \mathrm{O} & 2.60309900 & -1.87864800 & 0.00002300 \\ \mathrm{H} & 3.48036800 & -2.28372500 & 0.00001800 \\ \mathrm{H} & 0.26087200 & -1.47602600 & -0.00000400 \\ \mathrm{H} & 0.49980700 & 3.44413900 & 0.00001400 \\ \mathrm{H} & -1.67706000 & 2.34098700 & 0.00001400 \\ \mathrm{C} & -2.23339600 & -0.39008800 & -0.00000200 \\ \mathrm{C} & -2.32927097 & -1.27547427 & -1.25638536 \\ \mathrm{H} & -1.73819072 & -2.15655764 & -1.11776275 \\ \mathrm{H} & -3.34965256 & -1.55266101 & -1.42036380 \\ \mathrm{H} & -1.96658403 & -0.73237485 & -2.10397087 \\ \mathrm{C} & -2.32926300 & -1.27551984 & 1.25634985 \\ \mathrm{H} & -2.02481011 & -0.71233190 & 2.11368746 \\ \mathrm{H} & -3.33958529 & -1.60339452 & 1.38537336 \\ \mathrm{H} & -1.69000244 & -2.12603551 & 1.14290814 \\ \mathrm{H} & -3.06231017 & 0.28652216 & 0.00001270\end{array}$

\section{3-Cyclohexyl}

C

$-0.45577888-1.18896726-0.17882827$

$\begin{array}{llll}\text { C } & 0.20244374 & 0.03650358 & -0.17798554\end{array}$

C $\quad-0.51124522 \quad 1.21706016 \quad 0.00104801$

$\begin{array}{lllll}\text { C } & & -1.88342578 & 1.17168089 & 0.17924004\end{array}$

$\begin{array}{llll}\text { C } & -2.54187586 & -0.05149375 & 0.17859004\end{array}$

C $\quad-1.83002100-1.22936000-0.00000600$

C $\quad \begin{array}{llll}\text { C } & 1.67253955 & 0.13565900 & -0.36526909\end{array}$

$\begin{array}{lllll}\mathrm{O} & 2.29484852 & 1.16366633 & -0.37383670\end{array}$

$\begin{array}{llll}\mathrm{H} & 0.02626716 & 2.15497007 & -0.00276773\end{array}$

$\begin{array}{llll}\mathrm{O} & 2.26730788 & -1.06199059 & -0.52980257\end{array}$

H $\quad 3.21154355 \quad-0.89007948 \quad-0.64233217$

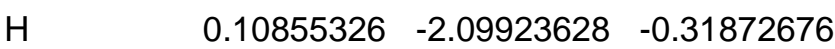

$\begin{array}{llll}\mathrm{H} & -2.44077169 & 2.08692184 & 0.31857530\end{array}$

$\begin{array}{llll}\mathrm{H} & -3.61350779 & -0.08608599 & 0.31781855\end{array}$

$\begin{array}{llll}\text { C } & -2.56419196 & -2.58309299 & -0.00000315\end{array}$

C $\quad \begin{array}{llll}\text { C } & -3.41700519 & -2.69850517 & 1.24705683\end{array}$

$\begin{array}{llll}\text { C } & -3.40621654 & -2.70434552 & -1.25413057\end{array}$

$\mathrm{H} \quad \begin{array}{llll}\mathrm{C} & -1.78468714 & -3.38963794 & 0.00517535\end{array}$

C $\quad-4.22260063 \quad-3.98168925 \quad 1.24795209$

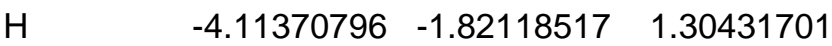

$\begin{array}{llll}\mathrm{H} & -2.76039904 & -2.66697097 & 2.15508002\end{array}$

$\begin{array}{llll}\text { C } & -4.21119408 & -3.98794020 & -1.25359029\end{array}$

H $\quad-4.10245628 \quad-1.82741999-1.32194445$

$\mathrm{H} \quad \begin{array}{llll}\mathrm{H} & -2.74202614 & -2.67797523 & -2.15668097\end{array}$

C $\quad-5.06331823 \quad-4.10481092-0.00632656$

$\begin{array}{llll}\mathrm{H} & -4.88772531 & -4.00697043 & 2.14986974\end{array}$

$\begin{array}{llll}\mathrm{H} & -3.52658280 & -4.85865473 & 1.31773307\end{array}$

H $\quad-4.86813758 \quad-4.01929311 \quad-2.16132821$ 


$\begin{array}{llll}H & -3.51391786 & -4.86480147 & -1.31181719 \\ H & -5.59556028 & -5.09137781 & -0.00622825 \\ H & -5.84482376 & -3.30009654 & -0.01178506\end{array}$

$\begin{array}{lrrr}\text { 3-Cyclobutyl } & & \\ \mathrm{C} & 0.51993400 & -0.62458100 & 0.00232700 \\ \mathrm{C} & 1.66501100 & 0.16710400 & 0.00017800 \\ \mathrm{C} & 1.56293700 & 1.55219200 & -0.00146300 \\ \mathrm{C} & 0.30760200 & 2.13862200 & -0.00092900 \\ \mathrm{C} & -0.83039600 & 1.34614200 & 0.00124800 \\ \mathrm{C} & -0.74408100 & -0.04712700 & 0.00287800 \\ \mathrm{C} & 3.02524200 & -0.42930800 & -0.00036900 \\ \mathrm{O} & 4.05022000 & 0.19846000 & -0.00209200 \\ \mathrm{H} & 2.46651100 & 2.14510400 & -0.00306800 \\ \mathrm{O} & 3.01445900 & -1.77719700 & 0.00126300 \\ \mathrm{H} & 3.93815300 & -2.06086800 & 0.00073300 \\ \mathrm{H} & 0.61970600 & -1.70131200 & 0.00363700 \\ \mathrm{H} & 0.21419700 & 3.21583200 & -0.00213800 \\ \mathrm{H} & -1.80500400 & 1.81981300 & 0.00174200 \\ \mathrm{C} & -1.97781900 & -0.90176900 & 0.00433500 \\ \mathrm{C} & -3.03824700 & -0.54116400 & 1.08593700 \\ \mathrm{C} & -3.03252400 & -0.55167800 & -1.08647800 \\ \mathrm{H} & -1.67928600 & -1.94810100 & 0.01013700 \\ \mathrm{C} & -3.96059100 & 0.04541800 & -0.00557100 \\ \mathrm{H} & -2.69895900 & 0.14707400 & 1.85792200 \\ \mathrm{H} & -3.45457000 & -1.42520100 & 1.56414500 \\ \mathrm{H} & -2.68899800 & 0.12914900 & -1.86312500 \\ \mathrm{H} & -3.44631800 & -1.44027700 & -1.55839200 \\ \mathrm{H} & -3.97646800 & 1.13391800 & -0.01094100 \\ \mathrm{H} & -4.98880700 & -0.30834300 & -0.00657400 \\ & & & \end{array}$

\section{3-Cyclopentyl}

$\begin{array}{lrrr}\text { C } & -0.78963500 & -0.49937300 & -0.00000900 \\ \text { C } & -2.03928700 & 0.11360800 & -0.00002300 \\ \text { C } & -2.14274800 & 1.49874200 & -0.00017000 \\ \text { C } & -0.98786600 & 2.26373900 & -0.00029100 \\ \text { C } & 0.25506700 & 1.64797200 & -0.00025900 \\ \text { C } & 0.37524500 & 0.25815000 & -0.00012800 \\ \text { C } & -3.29626600 & -0.67706400 & 0.00011300 \\ \text { O } & -4.40283000 & -0.20774800 & 0.00007300 \\ H & -3.12405300 & 1.95166400 & -0.00018600 \\ \text { O } & -3.08671700 & -2.00873700 & 0.00029700 \\ H & -3.95858500 & -2.42533100 & 0.00037100 \\ H & -0.72883600 & -1.57916300 & 0.00009300 \\ H & -1.05447200 & 3.34294000 & -0.00040800 \\ H & 1.14793500 & 2.26120000 & -0.00034100 \\ C & 1.72568700 & -0.40674900 & -0.00012100 \\ \text { C } & 2.62762200 & -0.05396800 & -1.19033300 \\ \text { C } & 2.62722100 & -0.05431900 & 1.19058300\end{array}$




$\begin{array}{lrrr}\mathrm{H} & 1.57271300 & -1.49021800 & -0.00044200 \\ \mathrm{C} & 4.01188600 & -0.57519000 & -0.77589000 \\ \mathrm{H} & 2.64886000 & 1.03188600 & -1.30750400 \\ \mathrm{H} & 2.27023500 & -0.47627000 & -2.12838100 \\ \mathrm{C} & 4.01287400 & -0.57233600 & 0.77590500 \\ \mathrm{H} & 2.27040200 & -0.47860600 & 2.12794200 \\ \mathrm{H} & 2.64639900 & 1.03136000 & 1.30947500 \\ \mathrm{H} & 4.15767900 & -1.58810700 & -1.14981200 \\ \mathrm{H} & 4.81332300 & 0.03326900 & -1.19054900 \\ \mathrm{H} & 4.16357500 & -1.58310600 & 1.15357600 \\ \mathrm{H} & 4.81239700 & 0.04107900 & 1.18695200\end{array}$

\section{3-Bn}

$C$

C

C

C

C

C

C

$\mathrm{O}$

$\mathrm{H}$

$\mathrm{O}$

$\mathrm{H}$

$\mathrm{H}$

$\mathrm{H}$

$\mathrm{H}$

C

$\mathrm{H}$

C

C

C

C

$\mathrm{H}$

C

$\mathrm{H}$

C

$\mathrm{H}$

$\mathrm{H}$

$\mathrm{H}$

$\mathrm{H}$

$$
\begin{array}{rrr}
0.35892752 & -1.18195972 & -0.09888126 \\
1.05497400 & 0.02960209 & 0.00863258 \\
0.36304667 & 1.21110321 & 0.30731911 \\
-0.97917025 & 1.18203409 & 0.49218602 \\
-1.67521713 & -0.02952725 & 0.38466938 \\
-0.98328957 & -1.21102863 & 0.08598446 \\
2.58022007 & 0.06263569 & -0.20144648 \\
3.20524108 & 1.15056914 & -0.10490573 \\
0.89449344 & 2.13615773 & 0.38940717 \\
3.28626885 & -1.14297826 & -0.50622528 \\
4.05476160 & -0.93670319 & -1.04331884 \\
0.88722961 & -2.08406229 & -0.32693356 \\
-1.50747252 & 2.08413689 & 0.72023703 \\
-2.73496628 & -0.05247885 & 0.53063158 \\
-1.74817629 & -2.54241466 & -0.03216605 \\
-1.24635764 & -3.18148312 & -0.72834816 \\
-3.18082324 & -2.26924001 & -0.52663379 \\
-3.81209804 & -1.05853159 & -0.21104037 \\
-3.85325715 & -3.23135949 & -1.29219286 \\
-5.11580629 & -0.80994213 & -0.66100709 \\
-3.29868080 & -0.32393310 & 0.37348320 \\
-5.15696531 & -2.98276994 & -1.74215977 \\
-3.37126549 & -4.15576297 & -1.53315394 \\
-5.78823909 & -1.77206037 & -1.42656870 \\
-5.59779841 & 0.11446084 & -0.42004497 \\
-5.67038406 & -3.71737011 & -2.32667991 \\
-6.78364831 & -1.58225574 & -1.77013045 \\
-1.78603168 & -3.01820328 & 0.92548324
\end{array}
$$

\section{3-SiMe}

$\begin{array}{lrrr}\text { C } & 0.58718900 & -0.33812000 & 0.00001100 \\ \text { C } & 1.86286700 & 0.21378000 & 0.00000700 \\ \text { C } & 2.02802900 & 1.59598200 & 0.00000900 \\ \text { C } & 0.91410400 & 2.41542700 & 0.00000700 \\ \text { C } & -0.35911600 & 1.85502700 & 0.00000300 \\ \text { C } & -0.54985400 & 0.47251900 & 0.00000500\end{array}$




$\begin{array}{lrrr}\mathrm{C} & 3.08451100 & -0.63072000 & -0.00000100 \\ \mathrm{O} & 4.21008000 & -0.20908000 & 0.00001700 \\ \mathrm{H} & 3.03017500 & 2.00162400 & 0.00001100 \\ \mathrm{O} & 2.81755400 & -1.95186100 & -0.00004000 \\ \mathrm{H} & 3.67044400 & -2.40610800 & -0.00005900 \\ \mathrm{H} & 0.49085500 & -1.41705400 & 0.00002300 \\ \mathrm{H} & 1.03321700 & 3.49025300 & 0.00001100 \\ \mathrm{H} & -1.21910500 & 2.51440800 & -0.00000400 \\ \mathrm{Si} & -2.26442200 & -0.31352500 & 0.00000800 \\ \mathrm{C} & -3.56090800 & 1.04148700 & 0.00039600 \\ \mathrm{H} & -4.56060500 & 0.60501000 & 0.00041100 \\ \mathrm{H} & -3.47457500 & 1.67486000 & 0.88432600 \\ \mathrm{H} & -3.47475700 & 1.67519700 & -0.88331200 \\ \mathrm{C} & -2.43612000 & -1.38167300 & -1.53302200 \\ \mathrm{H} & -1.66051100 & -2.14828200 & -1.56253800 \\ \mathrm{H} & -3.40446700 & -1.88394800 & -1.54977600 \\ \mathrm{H} & -2.34760900 & -0.78117200 & -2.43873600 \\ \mathrm{C} & -2.43581900 & -1.38237300 & 1.53261200 \\ \mathrm{H} & -2.34640200 & -0.78238800 & 2.43857700 \\ \mathrm{H} & -3.40445400 & -1.88407400 & 1.54970500 \\ \mathrm{H} & -1.66065600 & -2.14946400 & 1.56127500\end{array}$

$\begin{array}{lrrr}\text { 3-OPh } & & & \\ \text { C } & -1.40395200 & -0.66468000 & -0.11640200 \\ \text { C } & -2.53519700 & 0.13841000 & -0.04571200 \\ \text { C } & -2.44772200 & 1.51007400 & -0.25493800 \\ \text { C } & -1.21665800 & 2.07412200 & -0.54439900 \\ \text { C } & -0.07851200 & 1.28399400 & -0.62485600 \\ \text { C } & -0.17897600 & -0.08452000 & -0.40514500 \\ \text { C } & -3.87211900 & -0.43607800 & 0.25987500 \\ \text { O } & -4.88699500 & 0.20124600 & 0.34325600 \\ \text { H } & -3.34463500 & 2.10860600 & -0.18981900 \\ \text { O } & -3.84537800 & -1.77007500 & 0.44009400 \\ \text { H } & -4.75221100 & -2.04437800 & 0.63052900 \\ \text { H } & -1.46250200 & -1.73065200 & 0.04517400 \\ \text { H } & -1.13660500 & 3.13826700 & -0.71697000 \\ \text { O } & 0.89172400 & -0.93141700 & -0.52801000 \\ \text { C } & 2.14560500 & -0.50612300 & -0.15165500 \\ \text { C } & 3.20841900 & -0.87764400 & -0.95947100 \\ \text { C } & 2.35706600 & 0.20290400 & 1.02326200 \\ \text { C } & 4.49934900 & -0.53254900 & -0.58717400 \\ \text { H } & 3.00793700 & -1.43523000 & -1.86339600 \\ \text { C } & 3.65177800 & 0.54790700 & 1.38021700 \\ \text { H } & 1.51640300 & 0.47579600 & 1.64633800 \\ \mathrm{C} & 4.72614500 & 0.18386600 & 0.57939200 \\ \text { H } & 5.32993100 & -0.82306000 & -1.21565800 \\ \text { H } & 3.82023800 & 1.09884800 & 2.29537200 \\ \text { H } & 5.73273300 & 0.45435800 & 0.86505700 \\ \text { H } & 0.88254800 & 1.72131700 & -0.85731200\end{array}$




\begin{tabular}{|c|c|c|c|}
\hline \multicolumn{4}{|c|}{ 3-Ph } \\
\hline $\mathrm{C}$ & 0.79451300 & -0.33417900 & -0.05361400 \\
\hline C & 2.11348000 & 0.09908200 & -0.00269000 \\
\hline C & 2.40804800 & 1.44457300 & 0.18898200 \\
\hline C & 1.37269100 & 2.35238500 & 0.32994300 \\
\hline $\mathrm{C}$ & 0.05538400 & 1.91940900 & 0.27949200 \\
\hline C & -0.25325900 & 0.57278900 & 0.08687000 \\
\hline C & 3.25006000 & -0.84538100 & -0.15776600 \\
\hline $\mathrm{O}$ & 4.40961600 & -0.53138400 & -0.13086900 \\
\hline $\mathrm{H}$ & 3.44305200 & 1.75370000 & 0.22462600 \\
\hline $\mathrm{O}$ & 2.85849600 & -2.12219700 & -0.33478000 \\
\hline $\mathrm{H}$ & 3.66324800 & -2.64932900 & -0.42611500 \\
\hline $\mathrm{H}$ & 0.58466400 & -1.38027200 & -0.22571500 \\
\hline $\mathrm{H}$ & 1.58886000 & 3.39974800 & 0.48815700 \\
\hline $\mathrm{H}$ & -0.74822300 & 2.63178500 & 0.41336600 \\
\hline C & -1.66086000 & 0.11571700 & 0.03345300 \\
\hline C & -2.04049800 & -1.08998500 & 0.62223600 \\
\hline C & -2.63472000 & 0.88148700 & -0.60663000 \\
\hline C & -3.35816200 & -1.51780500 & 0.57375200 \\
\hline $\mathrm{H}$ & -1.30057500 & -1.68480900 & 1.14168900 \\
\hline C & -3.95242200 & 0.45379200 & -0.65685300 \\
\hline $\mathrm{H}$ & -2.35149900 & 1.80737500 & -1.09006300 \\
\hline C & -4.31898500 & -0.74737100 & -0.06614600 \\
\hline $\mathrm{H}$ & -3.63645000 & -2.45140000 & 1.04336400 \\
\hline $\mathrm{H}$ & -4.69288400 & 1.05589600 & -1.16556400 \\
\hline $\mathrm{H}$ & -5.34671300 & -1.08113100 & -0.10472200 \\
\hline \multicolumn{4}{|c|}{$3-F$} \\
\hline C & 0.39054500 & -0.86069000 & -0.00000300 \\
\hline C & -0.54655100 & 0.16665400 & -0.00000200 \\
\hline C & -0.14343500 & 1.49704400 & 0.00000900 \\
\hline $\mathrm{C}$ & 1.20718500 & 1.80489000 & 0.00001700 \\
\hline $\mathrm{C}$ & 2.15714300 & 0.79275500 & 0.00001600 \\
\hline C & 1.72656000 & -0.51982700 & 0.00000500 \\
\hline C & -2.00599900 & -0.12060700 & -0.00001100 \\
\hline $\mathrm{O}$ & -2.86583600 & 0.71742700 & -0.00001000 \\
\hline $\mathrm{H}$ & -0.89838700 & 2.26977500 & 0.00001000 \\
\hline $\mathrm{O}$ & -2.28231600 & -1.43761400 & -0.00002000 \\
\hline $\mathrm{H}$ & -3.24502600 & -1.52310000 & -0.00002600 \\
\hline $\mathrm{H}$ & 0.09282900 & -1.89833700 & -0.00001100 \\
\hline $\mathrm{H}$ & 1.52751800 & 2.83699500 & 0.00002500 \\
\hline $\mathrm{H}$ & 3.21699000 & 1.00369300 & 0.00002200 \\
\hline $\mathrm{F}$ & 2.64206600 & -1.49876100 & 0.00000400 \\
\hline
\end{tabular}

\begin{tabular}{|c|c|c|c|}
\hline $3-N_{3}$ & & & \\
\hline$C$ & 0.22267600 & -0.27905600 & -0.00000500 \\
\hline C & -1.09557800 & 0.16221300 & -0.00000100 \\
\hline C & -1.39319500 & 1.51880700 & 0.00000300 \\
\hline C & -0.35828600 & 2.44163300 & 0.00000100 \\
\hline
\end{tabular}




$\begin{array}{lrrr}\mathrm{C} & 0.95915500 & 2.01669700 & -0.00000300 \\ \mathrm{C} & 1.25026800 & 0.65561500 & -0.00000400 \\ \mathrm{C} & -2.22998800 & -0.80019300 & 0.00000100 \\ \mathrm{O} & -3.38941300 & -0.48833900 & 0.00001100 \\ \mathrm{H} & -2.42856400 & 1.82705300 & 0.00000600 \\ \mathrm{O} & -1.82990200 & -2.08567700 & -0.00001100 \\ \mathrm{H} & -2.62911200 & -2.62912500 & -0.00001000 \\ \mathrm{H} & 0.43085400 & -1.34027700 & -0.00000700 \\ \mathrm{H} & -0.57730700 & 3.50004400 & 0.00000300 \\ \mathrm{H} & 1.77801700 & 2.72227800 & -0.00000400 \\ \mathrm{~N} & 2.62652800 & 0.31177600 & -0.00000600 \\ \mathrm{~N} & 2.88304300 & -0.89219700 & 0.00000300 \\ \mathrm{~N} & 3.21190300 & -1.95988500 & 0.00001200\end{array}$

$\begin{array}{lrrr}\text { 3-Si(OMe) }{ }_{3} & & & \\ \mathrm{C} & -1.30537900 & -0.28291600 & -0.01374300 \\ \mathrm{C} & -2.62004600 & 0.16879000 & 0.00334200 \\ \mathrm{C} & -2.89218400 & 1.53330300 & 0.01850000 \\ \mathrm{C} & -1.84900100 & 2.44253500 & 0.01426900 \\ \mathrm{C} & -0.53613000 & 1.98860300 & -0.00220900 \\ \mathrm{C} & -0.24583300 & 0.62323200 & -0.01274300 \\ \mathrm{C} & -3.77238400 & -0.76807000 & 0.00149400 \\ \mathrm{O} & -4.92716500 & -0.43413800 & 0.01333000 \\ \mathrm{H} & -3.92375800 & 1.85709900 & 0.02937600 \\ \mathrm{O} & -3.40322900 & -2.06328000 & -0.01426700 \\ \mathrm{H} & -4.21730800 & -2.58380400 & -0.01411600 \\ \mathrm{H} & -2.05590000 & 3.50369100 & 0.02022900 \\ \mathrm{H} & -1.11391800 & -1.34729300 & -0.03701400 \\ \mathrm{H} & 0.27604500 & 2.70488400 & -0.01587400 \\ \mathrm{Si} & 1.50222900 & 0.02570300 & 0.00033800 \\ \mathrm{O} & 2.47356100 & 1.23914000 & -0.52321600 \\ \mathrm{O} & 1.62488300 & -1.27914900 & -0.98333800 \\ \mathrm{O} & 1.94569500 & -0.40519600 & 1.51932600 \\ \mathrm{C} & 2.52330100 & -2.35054500 & -0.77451900 \\ \mathrm{H} & 2.48877600 & -2.69639600 & 0.26061600 \\ \mathrm{H} & 2.23650900 & -3.17007300 & -1.43024000 \\ \mathrm{H} & 3.54885100 & -2.05612100 & -1.01523300 \\ \mathrm{C} & 3.25604500 & -0.27731000 & 2.03200700 \\ \mathrm{H} & 3.21899300 & -0.43051400 & 3.10861400 \\ \mathrm{H} & 3.92526300 & -1.02614400 & 1.59782500 \\ \mathrm{H} & 3.66185800 & 0.71645500 & 1.83069200 \\ \mathrm{C} & 3.65710500 & 1.04097700 & -1.27035700 \\ \mathrm{H} & 3.97537300 & 2.00144100 & -1.67043700 \\ \mathrm{H} & 4.45839800 & 0.64461100 & -0.64010500 \\ \mathrm{H} & 3.48668200 & 0.35170600 & -2.09999600\end{array}$

3-Cl

$\begin{array}{llll}\text { C } & 0.20187900 & -0.61564900 & -0.00000400\end{array}$

$\begin{array}{lllll}\text { C } & -0.93114000 & 0.19006800 & -0.00000200\end{array}$ 


$\begin{array}{lrrr}\mathrm{C} & -0.81745600 & 1.57547200 & 0.00000800 \\ \mathrm{C} & 0.43821200 & 2.15895900 & 0.00001700 \\ \mathrm{C} & 1.57868000 & 1.36765200 & 0.00001500 \\ \mathrm{C} & 1.44719800 & -0.01240200 & 0.00000500 \\ \mathrm{C} & -2.29748900 & -0.39842500 & -0.00001100 \\ \mathrm{O} & -3.31385200 & 0.24088500 & -0.00001000 \\ \mathrm{H} & -1.71822200 & 2.17228400 & 0.00001000 \\ \mathrm{O} & -2.29081700 & -1.74370500 & -0.00002000 \\ \mathrm{H} & -3.21387200 & -2.03021200 & -0.00002600 \\ \mathrm{H} & 0.11426500 & -1.69153500 & -0.00001200 \\ \mathrm{H} & 0.53684800 & 3.23526800 & 0.00002500 \\ \mathrm{H} & 2.56435800 & 1.81042900 & 0.00002200 \\ \mathrm{Cl} & 2.87262800 & -1.00398400 & 0.00000300\end{array}$

$\begin{array}{lrrr}\text { 3-Br } & & & \\ \mathrm{C} & -0.30917700 & -0.44914800 & -0.00000400 \\ \mathrm{C} & -1.54773300 & 0.18329700 & -0.00000200 \\ \mathrm{C} & -1.63663000 & 1.57039100 & 0.00000800 \\ \mathrm{C} & -0.47957500 & 2.33047100 & 0.00001600 \\ \mathrm{C} & 0.76455200 & 1.71373400 & 0.00001500 \\ \mathrm{C} & 0.83506100 & 0.32912300 & 0.00000400 \\ \mathrm{C} & -2.81419100 & -0.59734400 & -0.00001100 \\ \mathrm{O} & -3.91235000 & -0.11183900 & -0.00001000 \\ \mathrm{H} & -2.61484500 & 2.02959100 & 0.00000900 \\ \mathrm{O} & -2.61267700 & -1.92730400 & -0.00002100 \\ \mathrm{H} & -3.48446900 & -2.34462100 & -0.00002600 \\ \mathrm{H} & -0.24433600 & -1.52640300 & -0.00001200 \\ \mathrm{H} & -0.53846700 & 3.40971800 & 0.00002400 \\ \mathrm{H} & 1.67197100 & 2.30012100 & 0.00002100 \\ \mathrm{Br} & 2.52961500 & -0.51538300 & 0.00000200\end{array}$

$\begin{array}{lrrr}\text { 3-F } \mathbf{F}_{\mathbf{5}} \mathbf{P h} & & & \\ \mathrm{C} & 1.76650900 & -0.24629600 & -0.18446000 \\ \mathrm{C} & 3.10619000 & 0.08843600 & -0.03949600 \\ \mathrm{C} & 3.47394700 & 1.26557100 & 0.60246100 \\ \mathrm{C} & 2.49493100 & 2.10916400 & 1.09808500 \\ \mathrm{C} & 1.15543300 & 1.78025000 & 0.95333300 \\ \mathrm{C} & 0.78074000 & 0.60074900 & 0.31244800 \\ \mathrm{C} & 4.18853000 & -0.78812500 & -0.56089400 \\ \mathrm{O} & 5.36195700 & -0.55419200 & -0.45889500 \\ \mathrm{H} & 4.52484500 & 1.49841300 & 0.70141000 \\ \mathrm{O} & 3.72348900 & -1.89264300 & -1.17225200 \\ \mathrm{H} & 4.49349500 & -2.39430600 & -1.47156200 \\ \mathrm{H} & 1.49685500 & -1.16218400 & -0.68940600 \\ \mathrm{H} & 2.77120400 & 3.02531500 & 1.60034600 \\ \mathrm{H} & 0.39375200 & 2.43963900 & 1.34599900 \\ \mathrm{C} & -0.65062900 & 0.25135900 & 0.16011600 \\ \mathrm{C} & -1.13102800 & -1.01867600 & 0.46134700 \\ \mathrm{C} & -1.58613000 & 1.17572100 & -0.29226900\end{array}$




$\begin{array}{lrrr}C & -2.46504200 & -1.35705600 & 0.32333700 \\ C & -2.92486600 & 0.86197700 & -0.44113200 \\ C & -3.36697000 & -0.41166600 & -0.13110100 \\ F & -1.20443900 & 2.40788900 & -0.62272400 \\ F & -3.78325000 & 1.76927300 & -0.88630100 \\ F & -4.64546800 & -0.72374500 & -0.26812200 \\ F & -2.88537900 & -2.57604900 & 0.63195000 \\ F & -0.30517800 & -1.95521800 & 0.92205900\end{array}$

$\begin{array}{lrrr}\text { 3-OAC } & & & \\ \text { C } & -0.27981200 & -0.37903800 & -0.41219500 \\ \text { C } & -1.52119800 & 0.14334100 & -0.06798400 \\ \text { C } & -1.67064800 & 1.50112200 & 0.19130400 \\ \text { C } & -0.57311500 & 2.34157600 & 0.10276900 \\ \text { C } & 0.67165700 & 1.83024400 & -0.23744600 \\ \text { C } & 0.80796800 & 0.47301200 & -0.48329700 \\ \text { C } & -2.72659900 & -0.72332800 & 0.03003100 \\ \text { O } & -3.82535300 & -0.33183600 & 0.31371900 \\ \text { H } & -2.64961100 & 1.87627200 & 0.45302100 \\ \text { O } & -2.46508000 & -2.01682600 & -0.23196200 \\ \text { H } & -3.30011200 & -2.49661200 & -0.14912700 \\ H & -0.15758500 & -1.43070600 & -0.62861300 \\ H & -0.68370200 & 3.39933800 & 0.29496800 \\ \text { O } & 2.03829100 & -0.02234700 & -0.86862200 \\ \text { C } & 2.91162200 & -0.54901800 & 0.04589800 \\ \text { C } & 2.47221000 & -0.54711700 & 1.48422100 \\ \text { O } & 3.95395200 & -0.96920200 & -0.34679600 \\ \text { H } & 2.23364700 & 0.46410100 & 1.81153200 \\ \text { H } & 1.57172000 & -1.14768300 & 1.60740300 \\ \text { H } & 3.27811500 & -0.95668200 & 2.08233800 \\ \text { H } & 1.54053800 & 2.46887800 & -0.32204300\end{array}$

\section{3-CHO}

$0.33116700-0.53810600-0.00000400$

$\begin{array}{lll}-0.84354500 & 0.19591700 & -0.00000100\end{array}$

C $\quad-0.80342000-1.59041800-0.00001300$

$\begin{array}{lllll}\text { C } & -0.80342000 & 1.59041800 & 0.00001300\end{array}$

C $\quad 0.41172400 \quad 2.25220000 \quad 0.00002300$

$\begin{array}{llll}\text { C } & 1.59065200 & 1.51818600 & 0.00002000\end{array}$

C $\quad 1.55227500 \quad 0.12941800 \quad 0.00000700$

C $\quad-2.18011700-0.45854300-0.00001200$

$\begin{array}{lllll}\mathrm{O} & -3.22539100 & 0.13316200 & -0.00001000\end{array}$

$\mathrm{H} \quad-1.73866100 \quad 2.13303800 \quad 0.00001500$

$\begin{array}{llll}\text { O } & -2.10695800 & -1.79987400 & -0.00002500\end{array}$

$\mathrm{H} \quad-3.01394100-2.13401800-0.00003100$

$\mathrm{H} \quad 0.31819000 \quad-1.61899400 \quad-0.00001500$

$\begin{array}{llll}\mathrm{H} & 0.44157200 & 3.33238700 & 0.00003400\end{array}$

$\begin{array}{llll}\mathrm{H} & 2.54932400 & 2.02336100 & 0.00002900\end{array}$

$\begin{array}{llll}\text { C } & 2.82076300 & -0.63719200 & 0.00000400\end{array}$

$\begin{array}{llll}\mathrm{H} & 3.73831900 & -0.01942600 & 0.00001300\end{array}$ 


\begin{tabular}{|c|c|c|c|}
\hline \multirow{2}{*}{\multicolumn{4}{|c|}{$\begin{array}{l}\mathrm{O} \\
\text { 3-COPh }\end{array}$}} \\
\hline & & & \\
\hline C & -1.53391000 & -0.57264600 & -0.01085100 \\
\hline C & -2.67001200 & 0.22156000 & 0.02930500 \\
\hline C & -2.58430800 & 1.55140300 & 0.43458600 \\
\hline C & -1.35958600 & 2.08421900 & 0.79550300 \\
\hline C & -0.21634200 & 1.29763800 & 0.73393600 \\
\hline C & -0.29775500 & -0.03155800 & 0.32884500 \\
\hline C & -4.00957200 & -0.30556300 & -0.34428600 \\
\hline $\mathrm{O}$ & -5.02476900 & 0.33653800 & -0.31767900 \\
\hline $\mathrm{H}$ & -3.48784500 & 2.14444600 & 0.46307600 \\
\hline $\mathrm{O}$ & -3.98078400 & -1.59463700 & -0.72381500 \\
\hline $\mathrm{H}$ & -4.88851800 & -1.84543600 & -0.94102600 \\
\hline $\mathrm{H}$ & -1.59395800 & -1.61176300 & -0.30096900 \\
\hline $\mathrm{H}$ & -1.29094600 & 3.11121700 & 1.12516100 \\
\hline $\mathrm{H}$ & 0.73967800 & 1.71379500 & 1.02181500 \\
\hline C & 0.89363800 & -0.94074200 & 0.31616600 \\
\hline C & 2.24905700 & -0.37833000 & 0.02813000 \\
\hline C & 2.43709800 & 0.71572300 & -0.81261000 \\
\hline C & 3.35487600 & -1.02576900 & 0.57481500 \\
\hline C & 3.71980400 & 1.15972600 & -1.09956500 \\
\hline $\mathrm{H}$ & 1.58384100 & 1.20579100 & -1.26178700 \\
\hline C & 4.63300700 & -0.56830100 & 0.30484200 \\
\hline $\mathrm{H}$ & 3.19017300 & -1.88740200 & 1.20706100 \\
\hline C & 4.81622700 & 0.52476800 & -0.53397100 \\
\hline $\mathrm{H}$ & 3.86282900 & 1.99969500 & -1.76508100 \\
\hline $\mathrm{H}$ & 5.48830200 & -1.06489400 & 0.74174700 \\
\hline $\mathrm{H}$ & 5.81523300 & 0.87808700 & -0.75054400 \\
\hline $\mathrm{O}$ & 0.75403700 & -2.12143900 & 0.54043000 \\
\hline
\end{tabular}

$\begin{array}{lrrr}3-\mathrm{CO}_{2} \mathrm{Ph} & & & \\ \mathrm{C} & 1.59829800 & -0.18827700 & 0.02776400 \\ \mathrm{C} & 2.98729600 & -0.18065500 & 0.02797700 \\ \mathrm{C} & 3.68507600 & 1.01563100 & -0.10589400 \\ \mathrm{C} & 2.99469900 & 2.20945700 & -0.23981500 \\ \mathrm{C} & 1.60876800 & 2.20803100 & -0.23927400 \\ \mathrm{C} & 0.91102400 & 1.01151200 & -0.10604400 \\ \mathrm{C} & 3.77542700 & -1.43331700 & 0.16909200 \\ \mathrm{O} & 4.97540900 & -1.48566800 & 0.17798700 \\ \mathrm{H} & 4.76595200 & 0.98739500 & -0.10240800 \\ \mathrm{O} & 3.00504100 & -2.52960300 & 0.28874800 \\ \mathrm{H} & 3.60198700 & -3.28477900 & 0.37530300 \\ \mathrm{H} & 3.53517700 & 3.13937300 & -0.34417200 \\ \mathrm{H} & 1.04614500 & 3.12567600 & -0.34158200 \\ \mathrm{H} & 1.05913000 & -1.11716800 & 0.13163900 \\ \mathrm{C} & -0.57700200 & 1.07635800 & -0.11207600 \\ \mathrm{O} & -1.20818700 & 2.08942300 & -0.20348700 \\ \mathrm{O} & -1.12987600 & -0.15435200 & -0.00679200 \\ \mathrm{C} & -2.51538600 & -0.27844200 & 0.01416200\end{array}$




$\begin{array}{lrrr}\text { C } & -3.08083600 & -1.14546800 & -0.90238900 \\ \text { C } & -3.28249300 & 0.37978200 & 0.95994700 \\ \text { C } & -4.45155300 & -1.35892100 & -0.87256100 \\ \text { H } & -2.44505600 & -1.64306800 & -1.62097600 \\ \text { C } & -4.65116300 & 0.16008700 & 0.97746600 \\ H & -2.81468600 & 1.05582700 & 1.65997500 \\ \text { C } & -5.23853800 & -0.70641200 & 0.06487000 \\ H & -4.90199800 & -2.03637300 & -1.58456700 \\ H & -5.26100800 & 0.67073000 & 1.70971000 \\ H & -6.30644000 & -0.87220400 & 0.08608100\end{array}$

$\begin{array}{lrrr}3-\mathrm{CO}_{2} \mathrm{Me} & & & \\ \mathrm{C} & -0.77288200 & 0.59735200 & 0.00000000 \\ \mathrm{C} & 0.26156100 & -0.32947700 & 0.00000000 \\ \mathrm{C} & 1.57677200 & 0.11741400 & 0.00000000 \\ \mathrm{C} & 1.85726800 & 1.48035400 & 0.00000000 \\ \mathrm{C} & 0.82173700 & 2.40068600 & 0.00000000 \\ \mathrm{C} & -0.49253500 & 1.95989400 & 0.00000000 \\ \mathrm{H} & 2.89144100 & 1.79582200 & 0.00000000 \\ \mathrm{H} & 1.03803100 & 3.45949600 & 0.00000000 \\ \mathrm{H} & -1.31937800 & 2.65668500 & 0.00000000 \\ \mathrm{C} & -2.20389200 & 0.18119700 & 0.00000000 \\ \mathrm{O} & -3.12893800 & 0.94968500 & 0.00000100 \\ \mathrm{O} & -2.35410200 & -1.14796500 & 0.00000000 \\ \mathrm{C} & -3.70597000 & -1.60591100 & 0.00000000 \\ \mathrm{H} & -4.22515000 & -1.24568100 & 0.88574300 \\ \mathrm{H} & -4.22515100 & -1.24567600 & -0.88574000 \\ \mathrm{H} & -3.65230100 & -2.68919200 & -0.00000300 \\ \mathrm{H} & 0.04487500 & -1.38661700 & 0.00000000 \\ \mathrm{C} & 2.72341100 & -0.82831600 & 0.00000000 \\ \mathrm{O} & 2.34239100 & -2.11904200 & 0.00000000 \\ \mathrm{O} & 3.87874600 & -0.49944300 & 0.00000000 \\ \mathrm{H} & 3.15004100 & -2.64987900 & 0.00000000\end{array}$

\section{$3-\mathrm{CO}_{2} \mathrm{H}$}

$\mathrm{C}$

C

C

$\begin{array}{lll}-0.03149000 & -0.52428600 & -0.00000300\end{array}$

$\begin{array}{lll}-1.21232200 & 0.20297200 & -0.00001000\end{array}$

$\begin{array}{lll}-1.17935900 & 1.59510700 & -0.00000200\end{array}$

C $\quad 0.03488300 \quad 2.26015500 \quad 0.00001200$

C $\quad 1.21995400 \quad 1.53799400 \quad 0.00001900$

C $\quad 1.18488500 \quad 0.14740600 \quad 0.00001100$

C $\quad-2.54301100-0.46122800-0.00002500$

$\begin{array}{lllll}\mathrm{O} & -3.59331000 & 0.12179700 & -0.00003100\end{array}$

$\mathrm{H} \quad-2.11643700 \quad 2.13451600 \quad-0.00000800$

$\begin{array}{llll}\mathrm{O} & -2.46044700 & -1.80255600 & -0.00003100\end{array}$

$\mathrm{H} \quad-3.36535200-2.14213100-0.00004100$

$\mathrm{H} \quad-0.04060200 \quad-1.60458800-0.00000900$

$\begin{array}{llll}\mathrm{H} & 0.06074900 & 3.34047300 & 0.00001800\end{array}$

$\begin{array}{llll}\mathrm{H} & 2.17319800 & 2.04629400 & 0.00003000\end{array}$ 


$\begin{array}{lrrr}\mathrm{C} & 2.42736600 & -0.66906000 & 0.00001800 \\ \mathrm{O} & 2.45535300 & -1.86930200 & 0.00001000 \\ \mathrm{O} & 3.54704500 & 0.07834500 & 0.00003500 \\ \mathrm{H} & 4.29387100 & -0.53519700 & 0.00003700 \\ & & & \\ \mathbf{3 - C C l}{ }_{3} & & & \\ \mathrm{C} & -0.82725200 & -0.28809400 & 0.00008200 \\ \mathrm{C} & -2.08876000 & 0.28141500 & 0.00004400 \\ \mathrm{C} & -2.23880400 & 1.66670500 & 0.00004600 \\ \mathrm{C} & -1.11741700 & 2.47059200 & 0.00009200 \\ \mathrm{C} & 0.15409800 & 1.90438500 & 0.00013800 \\ \mathrm{C} & 0.30269300 & 0.52645200 & 0.00013900 \\ \mathrm{C} & -3.31999000 & -0.55357700 & 0.00000400 \\ \mathrm{O} & -4.43732800 & -0.11388100 & -0.00007600 \\ \mathrm{H} & -3.23622800 & 2.08309600 & 0.00001300 \\ \mathrm{O} & -3.06188700 & -1.87302600 & 0.00007200 \\ \mathrm{H} & -3.91469100 & -2.32795000 & 0.00004100 \\ \mathrm{H} & -0.72400900 & -1.36397900 & 0.00006100 \\ \mathrm{H} & -1.21927300 & 3.54641200 & 0.00009300 \\ \mathrm{H} & 1.02205900 & 2.54486700 & 0.00016700 \\ \mathrm{C} & 1.65857500 & -0.15342800 & 0.00002400 \\ \mathrm{Cl} & 3.01910900 & 0.98611800 & 0.00074500 \\ \mathrm{Cl} & 1.81167500 & -1.19124600 & 1.44760500 \\ \mathrm{Cl} & 1.81198200 & -1.18980800 & -1.44857200 \\ & & & \\ & & & \\ & & & \end{array}$

\section{3-COMe}

$\begin{array}{lrrr}\mathrm{C} & 0.00569400 & -0.46658400 & -0.00003300 \\ \mathrm{C} & -1.20825700 & 0.21161700 & -0.00001200 \\ \mathrm{C} & -1.23405100 & 1.60140200 & 0.00006300 \\ \mathrm{C} & -0.04478200 & 2.31462400 & 0.00006000 \\ \mathrm{C} & 1.16461800 & 1.63999300 & -0.00001400 \\ \mathrm{C} & 1.19799500 & 0.24651600 & -0.00003900 \\ \mathrm{C} & -2.50693400 & -0.51011900 & -0.00001900 \\ \mathrm{O} & -3.58398500 & 0.02101500 & 0.00010700 \\ \mathrm{H} & -2.19233600 & 2.10215800 & 0.00012700 \\ \mathrm{O} & -2.36568500 & -1.84971200 & -0.00018200 \\ \mathrm{H} & -3.25636000 & -2.22501500 & -0.00016200 \\ \mathrm{H} & -0.06285200 & 3.39530800 & 0.00016200 \\ \mathrm{H} & 2.10514400 & 2.17359900 & -0.00024800 \\ \mathrm{C} & 2.53411200 & -0.43585600 & -0.00001700 \\ \mathrm{O} & 3.55108000 & 0.21533300 & -0.00018300 \\ \mathrm{H} & 0.00342100 & -1.54670100 & -0.00004000 \\ \mathrm{C} & 2.56975100 & -1.94329400 & 0.00023400 \\ \mathrm{H} & 2.05891600 & -2.33660900 & 0.87986800 \\ \mathrm{H} & 2.05887000 & -2.33690400 & -0.87924100 \\ \mathrm{H} & 3.60503700 & -2.26871600 & 0.00025500\end{array}$

\section{$3-\mathrm{CF}_{3}$}

$\begin{array}{llll}\text { C } & -0.26808200 & -0.43926700 & 0.01707100\end{array}$ 


$\begin{array}{lrrr}\text { C } & -1.49243600 & 0.21370700 & 0.00286800 \\ \text { C } & -1.54786100 & 1.60427400 & -0.00479700 \\ \text { C } & -0.37787600 & 2.34266600 & 0.00171800 \\ \text { C } & 0.85128100 & 1.69621900 & 0.01534400 \\ \text { C } & 0.89840200 & 0.31068900 & 0.02395200 \\ \text { C } & -2.77741600 & -0.53611100 & -0.00177700 \\ \text { O } & -3.86262500 & -0.02292900 & -0.01477200 \\ \text { H } & -2.51681800 & 2.08357200 & -0.01366400 \\ \text { O } & -2.60605400 & -1.86938800 & 0.00976400 \\ \text { H } & -3.48617200 & -2.26904500 & 0.00552700 \\ \text { H } & -0.22676700 & -1.51911900 & 0.02789900 \\ \text { H } & -0.41762200 & 3.42244900 & -0.00150800 \\ \text { H } & 1.77010300 & 2.26502900 & 0.02473700 \\ \text { C } & 2.21795800 & -0.40746000 & 0.00095000 \\ \text { F } & 3.23035200 & 0.38484800 & 0.36689900 \\ \text { F } & 2.50492500 & -0.86974800 & -1.22471200 \\ \text { F } & 2.22059900 & -1.46539400 & 0.82060100\end{array}$

\section{3-C $\left(\mathrm{CF}_{3}\right)_{3}$}

$\mathrm{C}$

$-1.52105900-0.17802100$

0.04775900

C $\quad \begin{array}{llll}\text { C } & -2.79381600 & 0.37373800 & 0.05055800\end{array}$

C $\quad-2.97039100 \quad 1.75092500 \quad 0.07140600$

C $\quad-1.85797400 \quad 2.56872600 \quad 0.07367400$

C $\quad-0.58089500 \quad 2.02341300 \quad 0.07040500$

C $\quad-0.39293500 \quad 0.64219400 \quad 0.07817000$

$\begin{array}{llll}\text { C } & -4.00921200 & -0.48516700 & 0.02448800\end{array}$

$\begin{array}{llll}\text { O } & -5.13381800 & -0.06510500 & 0.02799600\end{array}$

H $\quad-3.97395200 \quad 2.15232600 \quad 0.07520200$

$\begin{array}{lllll}\mathrm{O} & -3.72624400 & -1.79872800 & -0.00416100\end{array}$

$\mathrm{H} \quad-4.56960600-2.27074500 \quad-0.02115200$

$\begin{array}{llll}\mathrm{H} & -1.43232000 & -1.25057800 & 0.01181200\end{array}$

$\begin{array}{llll}\mathrm{H} & -1.97147000 & 3.64331300 & 0.07332300\end{array}$

$\begin{array}{llll}\mathrm{H} & 0.25285800 & 2.70434800 & 0.05467200\end{array}$

$\begin{array}{llll}\text { C } & 1.00049900 & -0.00448300 & 0.01898500\end{array}$

$\begin{array}{llll}\text { C } & 2.14885300 & 1.04279600 & 0.03765000\end{array}$

$\begin{array}{llll}\text { C } & 1.24750700 & -0.97377200 & 1.21494600\end{array}$

C $\quad 1.11398500 \quad-0.80745000 \quad-1.31375500$

F $\quad \begin{array}{llll}\text { C } & 2.15024200 & 1.74139400 & -1.09898700\end{array}$

F $\quad 3.34085200 \quad 0.47178000 \quad 0.16607100$

F $\quad \begin{array}{llll}2.02710300 & 1.90501200 & 1.04489400\end{array}$

F $\quad 0.57005600 \quad-0.12889000 \quad-2.31785500$

F $\quad 2.37211400 \quad-1.07480000-1.64506000$

F $\quad \begin{array}{llll}0.46935600 & -1.97137800 & -1.21223200\end{array}$

F $\quad 2.28428600 \quad-1.77461000 \quad 0.97963500$

F $\quad 0.20250300 \quad-1.75310600 \quad 1.46972100$

F $\quad \begin{array}{llll}1.50211200 & -0.28044600 & 2.32156300\end{array}$

\section{3-CN}

C

$0.30831300-0.63708800-0.00000300$ 


$\begin{array}{lrrr}\mathrm{C} & -0.82798500 & 0.15885200 & -0.00000100 \\ \mathrm{C} & -0.71779300 & 1.54547400 & 0.00000800 \\ \mathrm{C} & 0.53226500 & 2.14249100 & 0.00001600 \\ \mathrm{C} & 1.67494000 & 1.35882500 & 0.00001400 \\ \mathrm{C} & 1.56079800 & -0.03052100 & 0.00000500 \\ \mathrm{C} & -2.19413100 & -0.43285800 & -0.00001000 \\ \mathrm{O} & -3.20894000 & 0.20768400 & -0.00000800 \\ \mathrm{H} & -1.62335200 & 2.13600400 & 0.00001000 \\ \mathrm{O} & -2.18135400 & -1.77629900 & -0.00001900 \\ \mathrm{H} & -3.10203600 & -2.07109300 & -0.00002400 \\ \mathrm{H} & 0.22406100 & -1.71353700 & -0.00001100 \\ \mathrm{H} & 0.61894600 & 3.21946900 & 0.00002400 \\ \mathrm{C} & 2.74513000 & -0.84221700 & 0.00000300 \\ \mathrm{~N} & 3.69412500 & -1.48723900 & 0.00000100 \\ \mathrm{H} & 2.65663400 & 1.81100500 & 0.00002000\end{array}$

\section{3-SO ${ }_{2} \mathrm{Ph}$}

$\mathrm{C}$

C

C

C

C

C

C

$\mathrm{O}$

$\mathrm{H}$

$\mathrm{O}$

$\mathrm{H}$

$\mathrm{H}$

$\mathrm{H}$

$\mathrm{H}$

$\mathrm{S}$

O

O

C

C

C

C

$\mathrm{H}$

C

$\mathrm{H}$

C

$\mathrm{H}$

$\mathrm{H}$

$\mathrm{H}$

$\begin{array}{rrr}-1.43246500 & 0.03876600 & 0.44867900 \\ -2.60890900 & 0.07128400 & -0.28939800 \\ -2.82521900 & -0.85043900 & -1.30945700 \\ -1.87048100 & -1.81255100 & -1.59572300 \\ -0.68967000 & -1.85460100 & -0.86729700 \\ -0.48718700 & -0.92489800 & 0.14112600 \\ -3.67396700 & 1.07423800 & -0.01783100 \\ -4.71145800 & 1.14163700 & -0.61827400 \\ -3.75316100 & -0.79922700 & -1.86194100 \\ -3.35748900 & 1.91568800 & 0.98090100 \\ -4.10303500 & 2.52119400 & 1.08984600 \\ -1.26130100 & 0.73385700 & 1.25829900 \\ -2.04613400 & -2.53453200 & -2.38030100 \\ 0.06354500 & -2.60732600 & -1.05634200 \\ 1.03361500 & -0.97186700 & 1.07223500 \\ 0.77013100 & -0.36835500 & 2.36352400 \\ 1.56648400 & -2.31576700 & 0.96034100 \\ 2.11501800 & 0.11872500 & 0.17261500 \\ 2.12937200 & 1.46693600 & 0.49575200 \\ 2.91297000 & -0.40478800 & -0.83293300 \\ 2.96296100 & 2.31413300 & -0.21847100 \\ 1.51206500 & 1.83139900 & 1.30528500 \\ 3.74264200 & 0.45280800 & -1.53948200 \\ 2.89267300 & -1.46676100 & -1.03520800 \\ 3.76350900 & 1.80761600 & -1.23433900 \\ 2.99334600 & 3.36741200 & 0.02256100 \\ 4.37769700 & 0.06285600 & -2.32248100 \\ 4.41365800 & 2.47200300 & -1.78685900\end{array}$

\section{3- $\mathrm{SO}_{2} \mathrm{Me}$}

$\mathrm{C}$

C

$\begin{array}{rrr}-0.50398200 & -0.40319000 & -0.07249000 \\ -1.75312900 & 0.20054200 & 0.00058100\end{array}$ 


$\begin{array}{lrrr}\text { C } & -1.86185700 & 1.58701000 & 0.05453400 \\ \mathrm{C} & -0.72565300 & 2.37939500 & 0.03242100 \\ \mathrm{C} & 0.52887600 & 1.78888100 & -0.03711700 \\ \mathrm{C} & 0.61990800 & 0.40593200 & -0.08248500 \\ \mathrm{C} & -3.00857300 & -0.59896500 & 0.01421200 \\ \mathrm{O} & -4.11123400 & -0.12760800 & 0.06889700 \\ \mathrm{H} & -2.84938200 & 2.02430400 & 0.10567100 \\ \mathrm{O} & -2.78505500 & -1.92240700 & -0.03814500 \\ \mathrm{H} & -3.64784600 & -2.35836700 & -0.03225100 \\ \mathrm{H} & -0.40443600 & -1.47743000 & -0.14155600 \\ \mathrm{H} & -0.81444000 & 3.45593100 & 0.06120600 \\ \mathrm{H} & 1.43216600 & 2.38258700 & -0.07782300 \\ \mathrm{~S} & 2.23063200 & -0.35462800 & -0.16216200 \\ \mathrm{O} & 2.08498000 & -1.64416000 & -0.80781000 \\ \mathrm{O} & 3.16130300 & 0.63096800 & -0.67706900 \\ \mathrm{C} & 2.63908600 & -0.64700700 & 1.54671400 \\ \mathrm{H} & 3.62255000 & -1.11229900 & 1.53708400 \\ \mathrm{H} & 2.66619300 & 0.30696400 & 2.06588000 \\ \mathrm{H} & 1.89707700 & -1.31759100 & 1.97117000\end{array}$

$\begin{array}{lrrr}\text { 3-NO } & \\ \mathrm{C} & & & \\ \mathrm{C} & 0.00155200 & -0.53596100 & -0.00000500 \\ \mathrm{C} & -1.16806700 & 0.21156400 & -0.00000200 \\ \mathrm{C} & -1.11702800 & 1.60214600 & 0.00001200 \\ \mathrm{C} & 0.10463400 & 2.25596400 & 0.00002200 \\ \mathrm{C} & 1.28325900 & 1.52510900 & 0.00002000 \\ \mathrm{C} & 1.20345000 & 0.14413300 & 0.00000600 \\ \mathrm{O} & -2.50761700 & -0.43868700 & -0.00001300 \\ \mathrm{H} & -3.54820900 & 0.15913700 & -0.00001100 \\ \mathrm{O} & -2.04786100 & 2.15202700 & 0.00001400 \\ \mathrm{H} & -2.43597200 & -1.77922700 & -0.00002600 \\ \mathrm{H} & -3.34240100 & -2.11543300 & -0.00003200 \\ \mathrm{H} & -0.01442900 & -1.61454800 & -0.00001600 \\ \mathrm{H} & 0.14282800 & 3.33555500 & 0.00003300 \\ \mathrm{~N} & 2.25205000 & 2.00092200 & 0.00002800 \\ \mathrm{O} & 2.45714300 & -0.63806600 & 0.00000300 \\ \mathrm{O} & 2.36215000 & -1.84381600 & -0.00000900 \\ & 3.49812100 & -0.02080400 & 0.00001200\end{array}$

\section{$3-\mathrm{COCF}_{3}$}

$\mathrm{C}$

C $\quad-1.91850900 \quad 0.13676900 \quad-0.00003200$

C $\quad-2.38404900 \quad 1.44655900 \quad 0.00017100$

C $\quad-1.48594800 \quad 2.50334700 \quad 0.00015000$

C $\quad-0.12627300 \quad 2.24888500 \quad-0.00005800$

C $\quad 0.34534800 \quad 0.93553800 \quad-0.00015700$

C $\quad-2.92472000 \quad-0.95975500 \quad-0.00003500$

$\begin{array}{llll}\mathrm{O} & -4.11283800 & -0.78858500 & 0.00027300\end{array}$ 


$\begin{array}{lrrr}H & -3.45218100 & 1.61509600 & 0.00034300 \\ \mathrm{O} & -2.36669200 & -2.18199800 & -0.00042400 \\ \mathrm{H} & -3.08825500 & -2.82529300 & -0.00037600 \\ \mathrm{H} & -1.84690900 & 3.52177900 & 0.00037800 \\ \mathrm{H} & 0.59367000 & 3.05564100 & -0.00054100 \\ \mathrm{C} & 1.81822900 & 0.74746200 & -0.00018100 \\ \mathrm{O} & 2.60836100 & 1.64831000 & -0.00040300 \\ \mathrm{C} & 2.36801300 & -0.70225400 & 0.00012600 \\ \mathrm{H} & -0.21893700 & -1.15196200 & -0.00020100 \\ \mathrm{~F} & 1.94335300 & -1.36733500 & -1.08063200 \\ \mathrm{~F} & 1.94314700 & -1.36697600 & 1.08102000 \\ \mathrm{~F} & 3.68654600 & -0.71228700 & 0.00025300\end{array}$

\begin{tabular}{|c|c|c|c|}
\hline \multicolumn{4}{|c|}{$3-\mathrm{SO}_{2} \mathrm{~F}$} \\
\hline C & -0.49216500 & -0.41423000 & -0.05565700 \\
\hline C & -1.73459000 & 0.20338000 & -0.01002300 \\
\hline C & -1.82999700 & 1.59132200 & 0.01679400 \\
\hline C & -0.68746600 & 2.37528600 & -0.00443900 \\
\hline C & 0.56236300 & 1.77574000 & -0.04953800 \\
\hline C & 0.63450100 & 0.39069700 & -0.06937200 \\
\hline C & -2.99850500 & -0.58517000 & 0.00577800 \\
\hline $\mathrm{O}$ & -4.09484300 & -0.09962100 & 0.04471100 \\
\hline $\mathrm{H}$ & -2.81436100 & 2.03764800 & 0.04885800 \\
\hline $\mathrm{O}$ & -2.78424400 & -1.90923600 & -0.02733800 \\
\hline $\mathrm{H}$ & -3.64851900 & -2.34251300 & -0.01546300 \\
\hline $\mathrm{H}$ & -0.40121800 & -1.49028200 & -0.09021100 \\
\hline $\mathrm{H}$ & -0.76722400 & 3.45253900 & 0.00885100 \\
\hline $\mathrm{H}$ & 1.47044600 & 2.36148800 & -0.08018400 \\
\hline S & 2.22003700 & -0.37520200 & -0.12680800 \\
\hline $\mathrm{O}$ & 2.11403300 & -1.71007000 & -0.62153600 \\
\hline $\mathrm{O}$ & 3.20118500 & 0.54552800 & -0.60505000 \\
\hline $\mathrm{F}$ & 2.49182100 & -0.51673500 & 1.42550300 \\
\hline
\end{tabular}

\begin{tabular}{|c|c|c|c|}
\hline \multicolumn{4}{|c|}{$3-\mathrm{SO}_{2} \mathrm{CF}_{3}$} \\
\hline C & -1.05765400 & -0.29620500 & -0.35853000 \\
\hline C & -2.30959700 & 0.17262500 & 0.01513300 \\
\hline C & -2.48843600 & 1.51288400 & 0.34520700 \\
\hline C & -1.42272100 & 2.39809300 & 0.30325000 \\
\hline C & -0.16471300 & 1.94737500 & -0.06760400 \\
\hline C & -0.00668900 & 0.60652700 & -0.39006400 \\
\hline C & -3.49643100 & -0.72612200 & 0.07208400 \\
\hline $\mathrm{O}$ & -4.59742500 & -0.36858500 & 0.38728200 \\
\hline $\mathrm{H}$ & -3.47809800 & 1.84287500 & 0.62989500 \\
\hline $\mathrm{O}$ & -3.20580600 & -1.99089400 & -0.26800600 \\
\hline $\mathrm{H}$ & -4.02390400 & -2.50249800 & -0.20630800 \\
\hline $\mathrm{H}$ & -0.90203600 & -1.33060400 & -0.63017800 \\
\hline $\mathrm{H}$ & -1.57028400 & 3.43842900 & 0.55416300 \\
\hline $\mathrm{H}$ & 0.68415700 & 2.61501600 & -0.12195600 \\
\hline $\mathrm{S}$ & 1.59627600 & 0.02747500 & -0.86055100 \\
\hline
\end{tabular}




$\begin{array}{lrrr}\text { O } & 1.47263800 & -1.19495400 & -1.61405900 \\ \text { O } & 2.40185900 & 1.13710500 & -1.30592600 \\ \text { C } & 2.31065800 & -0.47404000 & 0.78437400 \\ F & 3.53077100 & -0.93600100 & 0.59531900 \\ F & 2.35347300 & 0.57305900 & 1.59521800 \\ F & 1.55943500 & -1.41606000 & 1.33454900\end{array}$

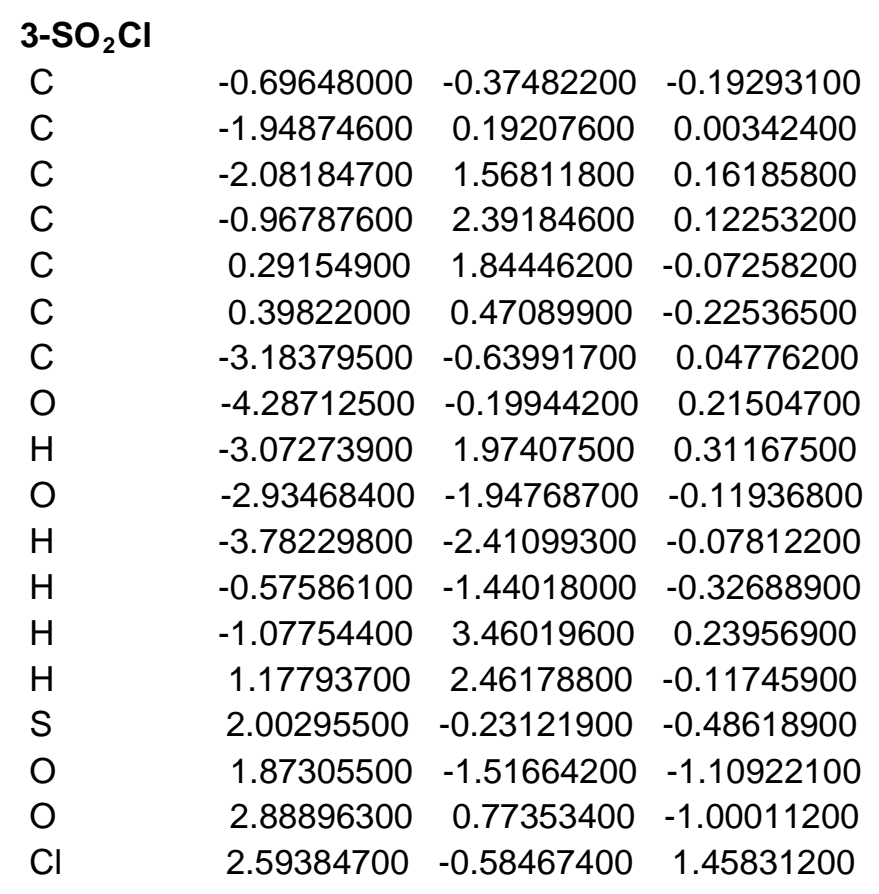

C. Cartesian Coordinates of geometry optimized ortho-substituted benzoic acids

\begin{tabular}{|c|c|c|c|}
\hline \multicolumn{4}{|c|}{$2-\mathrm{NMe}_{2}$} \\
\hline C & 0.03062600 & -0.70938100 & -0.02663300 \\
\hline C & 0.51793100 & 0.61573200 & 0.02628100 \\
\hline C & 1.88737000 & 0.85598300 & 0.11977500 \\
\hline C & 2.80645000 & -0.17461600 & 0.08402500 \\
\hline C & 2.33753800 & -1.47506100 & -0.04459800 \\
\hline C & 0.98145200 & -1.74093200 & -0.09024600 \\
\hline C & -0.31202000 & 1.83855200 & -0.12334400 \\
\hline $\mathrm{O}$ & -0.02964900 & 2.91390900 & 0.33913200 \\
\hline $\mathrm{H}$ & 2.21024500 & 1.88586200 & 0.18485000 \\
\hline $\mathrm{O}$ & -1.39680700 & 1.67767700 & -0.90771600 \\
\hline $\mathrm{H}$ & -1.81244400 & 2.54851600 & -0.97054600 \\
\hline $\mathrm{H}$ & 3.86528800 & 0.03104200 & 0.13855300 \\
\hline $\mathrm{H}$ & 3.03512000 & -2.30120200 & -0.08600400 \\
\hline $\mathrm{H}$ & 0.65230100 & -2.76745400 & -0.13823500 \\
\hline $\mathrm{N}$ & -1.32199000 & -0.99691000 & -0.00673900 \\
\hline C & -1.76222300 & -2.33248500 & -0.33837800 \\
\hline $\mathrm{H}$ & -1.57721100 & -3.05463800 & 0.46831700 \\
\hline $\mathrm{H}$ & -2.83475900 & -2.30331800 & -0.52528800 \\
\hline C & -2.20361000 & -0.32133500 & 0.92926100 \\
\hline
\end{tabular}




$\begin{array}{lrrr}H & -3.07689300 & 0.08880000 & 0.42110800 \\ H & -2.54003200 & -1.02803800 & 1.69551300 \\ H & -1.68630600 & 0.48894700 & 1.43500700 \\ H & -1.27081600 & -2.68158500 & -1.24429500\end{array}$

$\begin{array}{lrrr}\text { 2-NEt }{ }_{2} & & & \\ \mathrm{C} & -0.03912300 & -0.57529300 & -0.07054000 \\ \mathrm{C} & 1.02041800 & 0.28517800 & 0.20829400 \\ \mathrm{C} & 2.32847400 & -0.18931500 & 0.24034000 \\ \mathrm{C} & 2.58708000 & -1.53523100 & 0.04799500 \\ \mathrm{C} & 1.53475300 & -2.40551800 & -0.20591600 \\ \mathrm{C} & 0.23716100 & -1.92508000 & -0.27766700 \\ \mathrm{C} & 0.81766300 & 1.74627700 & 0.43946800 \\ \mathrm{O} & 1.39486100 & 2.60619600 & -0.17024800 \\ \mathrm{H} & 3.13436600 & 0.50881700 & 0.42187000 \\ \mathrm{O} & -0.01915900 & 2.01979200 & 1.45170600 \\ \mathrm{H} & -0.06976600 & 2.98350600 & 1.51923700 \\ \mathrm{H} & 3.60247000 & -1.90336100 & 0.09031300 \\ \mathrm{H} & 1.72824600 & -3.45799100 & -0.36358000 \\ \mathrm{H} & -0.57630000 & -2.60097200 & -0.50786900 \\ \mathrm{~N} & -1.36180500 & -0.04716500 & -0.21657800 \\ \mathrm{C} & -1.77190300 & 0.01746700 & -1.62309400 \\ \mathrm{H} & -1.83289900 & -0.99257300 & -2.05886400 \\ \mathrm{H} & -2.78209000 & 0.42947600 & -1.63345900 \\ \mathrm{C} & -2.36376700 & -0.73494400 & 0.59755600 \\ \mathrm{H} & -3.30651700 & -0.21047000 & 0.43504200 \\ \mathrm{H} & -2.52112900 & -1.77033700 & 0.25721500 \\ \mathrm{C} & -0.85791700 & 0.88969300 & -2.46502400 \\ \mathrm{H} & 0.14961100 & 0.47871100 & -2.52338300 \\ \mathrm{H} & -1.24868900 & 0.96560500 & -3.47906100 \\ \mathrm{H} & -0.78861700 & 1.89221100 & -2.04349900 \\ \mathrm{C} & -2.01951300 & -0.71694200 & 2.07608100 \\ \mathrm{H} & -2.82720900 & -1.16538800 & 2.65337300 \\ \mathrm{H} & -1.10817400 & -1.27922500 & 2.27894100 \\ \mathrm{H} & -1.86623700 & 0.30648700 & 2.41314300\end{array}$

\begin{tabular}{|c|c|c|c|}
\hline $2-\mathrm{NH}_{2}$ & & & \\
\hline $\mathrm{C}$ & -0.47206600 & 0.97557700 & -0.01374600 \\
\hline C & 0.19385800 & -0.26806100 & -0.00621100 \\
\hline C & -0.55373700 & -1.45262000 & 0.00377100 \\
\hline C & -1.92856600 & -1.44624700 & 0.01466500 \\
\hline C & -2.58815400 & -0.21636200 & 0.01426200 \\
\hline C & -1.87915200 & 0.96117300 & -0.00052500 \\
\hline C & 1.65856200 & -0.45365600 & -0.00325400 \\
\hline $\mathrm{O}$ & 2.22036800 & -1.51673300 & -0.04156800 \\
\hline $\mathrm{H}$ & -0.00190900 & -2.38196700 & 0.00606900 \\
\hline $\mathrm{O}$ & 2.38036100 & 0.69574000 & 0.05763300 \\
\hline $\mathrm{H}$ & 3.30896700 & 0.42709700 & 0.05555800 \\
\hline
\end{tabular}




$\begin{array}{rrrr}H & -2.48425100 & -2.37194300 & 0.02500500 \\ H & -3.66953000 & -0.18098800 & 0.02454200 \\ H & -2.40194500 & 1.90920500 & -0.00892900 \\ \mathrm{~N} & 0.15803500 & 2.18892900 & -0.07438500 \\ \mathrm{H} & 1.14360700 & 2.24193400 & 0.10484500 \\ \mathrm{H} & -0.39149600 & 3.00328300 & 0.13130900\end{array}$

\begin{tabular}{|c|c|c|c|}
\hline \multicolumn{4}{|c|}{ 2-0-iPr } \\
\hline C & -0.00713600 & 0.44352900 & -0.12774400 \\
\hline C & 1.24458900 & -0.18902900 & -0.01277900 \\
\hline C & 2.40145600 & 0.58500700 & 0.02128700 \\
\hline C & 2.35171800 & 1.96532600 & -0.02546700 \\
\hline C & 1.11454600 & 2.58397500 & -0.12016800 \\
\hline C & -0.05205500 & 1.83922300 & -0.17388700 \\
\hline $\mathrm{H}$ & 3.25996200 & 2.54848100 & 0.01300800 \\
\hline C & 1.46090600 & -1.66017000 & 0.07104200 \\
\hline $\mathrm{O}$ & 2.52643000 & -2.18147500 & -0.14032100 \\
\hline $\mathrm{H}$ & 1.04814200 & 3.66320800 & -0.15672200 \\
\hline $\mathrm{H}$ & 3.34555300 & 0.06340500 & 0.09092800 \\
\hline $\mathrm{O}$ & 0.39281300 & -2.38021400 & 0.44668400 \\
\hline $\mathrm{H}$ & 0.69387700 & -3.29863300 & 0.48149400 \\
\hline $\mathrm{H}$ & -0.99709400 & 2.35187200 & -0.25523100 \\
\hline $\mathrm{O}$ & -1.09799800 & -0.33635700 & -0.23379800 \\
\hline C & -2.40843500 & 0.23360200 & -0.25475700 \\
\hline $\mathrm{H}$ & -2.42172300 & 1.07066800 & -0.95754600 \\
\hline C & -3.31670800 & -0.86199700 & -0.77433600 \\
\hline $\mathrm{H}$ & -3.27199500 & -1.72336400 & -0.10833600 \\
\hline $\mathrm{H}$ & -4.34515400 & -0.50576100 & -0.82015600 \\
\hline $\mathrm{H}$ & -3.00720000 & -1.17640900 & -1.76913200 \\
\hline C & -2.81001500 & 0.69271800 & 1.13700900 \\
\hline $\mathrm{H}$ & -3.80423900 & 1.13829400 & 1.11187600 \\
\hline $\mathrm{H}$ & -2.82987400 & -0.16614600 & 1.80795100 \\
\hline $\mathrm{H}$ & -2.11341200 & 1.42565900 & 1.54015000 \\
\hline
\end{tabular}

$\begin{array}{lrrr}\text { 2-OBn } & & & \\ \text { C } & -1.35251100 & -0.61273800 & 0.01226400 \\ \text { C } & -2.34383700 & 0.38590900 & -0.00955800 \\ \text { C } & -3.68413200 & 0.00798200 & -0.01397800 \\ \text { C } & -4.06588800 & -1.32015800 & 0.01379300 \\ \text { C } & -3.08299900 & -2.29666800 & 0.04072100 \\ \text { C } & -1.74067300 & -1.95311900 & 0.03772100 \\ \text { H } & -5.11193100 & -1.58888900 & 0.01412700 \\ \text { C } & -2.10020500 & 1.85476900 & -0.03256100 \\ \text { O } & -2.97844000 & 2.66485300 & -0.18448400 \\ \text { H } & -3.35587300 & -3.34324100 & 0.06220300 \\ \text { H } & -4.42041900 & 0.79860300 & -0.03847300 \\ \text { O } & -0.82570300 & 2.23911900 & 0.14827700 \\ \text { H } & -0.84102800 & 3.20612100 & 0.12149600 \\ \text { H } & -0.99806800 & -2.73462700 & 0.05301200\end{array}$




$\begin{array}{lrrr}\mathrm{O} & -0.06087500 & -0.23340500 & -0.00537800 \\ \mathrm{C} & 0.94283000 & -1.22549700 & 0.01152000 \\ \mathrm{H} & 0.83913900 & -1.84337300 & 0.90977800 \\ \mathrm{H} & 0.83764300 & -1.87875100 & -0.86083700 \\ \mathrm{C} & 2.29525100 & -0.56653700 & -0.00209900 \\ \mathrm{C} & 3.42593800 & -1.37422700 & -0.08077600 \\ \mathrm{C} & 2.44223300 & 0.81154000 & 0.07134500 \\ \mathrm{C} & 4.69204100 & -0.81275600 & -0.08419000 \\ \mathrm{H} & 3.31329200 & -2.45063900 & -0.14099800 \\ \mathrm{C} & 3.71378600 & 1.37292300 & 0.06632300 \\ \mathrm{H} & 1.56057500 & 1.43350900 & 0.12946200 \\ \mathrm{C} & 4.83883500 & 0.56694900 & -0.01042300 \\ \mathrm{H} & 5.56389400 & -1.44957300 & -0.14605300 \\ \mathrm{H} & 3.82300000 & 2.44759200 & 0.12263400 \\ \mathrm{H} & 5.82589900 & 1.00850200 & -0.01429300\end{array}$

$\begin{array}{lrrr}2-O H & & & \\ \text { C } & 0.48703700 & 0.95920400 & 0.00000200 \\ \text { C } & -0.20894200 & -0.25652000 & 0.00000000 \\ \text { C } & 0.52074700 & -1.44680700 & -0.00000300 \\ \text { C } & 1.90059100 & -1.45566200 & -0.00000500 \\ \text { C } & 2.58096500 & -0.24367200 & -0.00000200 \\ \text { C } & 1.88189800 & 0.94746400 & 0.00000200 \\ \text { C } & -1.68939800 & -0.40811700 & 0.00000100 \\ \text { O } & -2.24305600 & -1.47798700 & 0.00001500 \\ \text { H } & -0.04264600 & -2.36911100 & -0.00000400 \\ \text { O } & -2.37845600 & 0.74099200 & -0.00001700 \\ \text { H } & -3.31308100 & 0.49254600 & -0.00001600 \\ \text { H } & 2.44156600 & -2.39036000 & -0.00000800 \\ \text { H } & 3.66239100 & -0.22431100 & -0.00000300 \\ \text { H } & 2.41285400 & 1.89206300 & 0.00000500 \\ \text { O } & -0.18407900 & 2.13265200 & 0.00000800 \\ \text { H } & 0.44625500 & 2.85858200 & 0.00001300\end{array}$

$\begin{array}{lrrr}\text { 2-OMe } & & & \\ \text { C } & -0.39407800 & 0.70900500 & -0.26544500 \\ \text { C } & 0.04804000 & -0.59765800 & -0.04231800 \\ \text { C } & -0.89167500 & -1.60235500 & 0.18986100 \\ \text { C } & -2.24251700 & -1.31711700 & 0.23818500 \\ \text { C } & -2.67356100 & -0.01180100 & 0.03144000 \\ \text { C } & -1.75569500 & 0.99092400 & -0.22606500 \\ \text { H } & -2.95683100 & -2.10516300 & 0.42855100 \\ \text { C } & 1.47497000 & -1.02245800 & -0.04821900 \\ \text { O } & 1.82911500 & -2.15839700 & -0.22249100 \\ \text { O } & 0.47282400 & 1.70834600 & -0.59707600 \\ \text { C } & 0.79446800 & 2.58473100 & 0.47347000 \\ \text { H } & -0.10590200 & 3.06275500 & 0.86649400 \\ \text { H } & 1.30078500 & 2.04044500 & 1.27190200 \\ \text { H } & 1.46031600 & 3.34074500 & 0.06723000\end{array}$




$\begin{array}{rrrr}H & -3.72926200 & 0.22214400 & 0.05618500 \\ H & -0.52778200 & -2.60999400 & 0.33421100 \\ \mathrm{O} & 2.35347300 & -0.03859500 & 0.20774700 \\ \mathrm{H} & 3.23018700 & -0.44666000 & 0.18932800 \\ \mathrm{H} & -2.07451700 & 2.00526500 & -0.42479800\end{array}$

$\begin{array}{lrrr}2-\text { OSiMe }_{3} & & & \\ \mathrm{C} & 0.54204800 & -0.58600700 & -0.47841000 \\ \mathrm{C} & 1.54148000 & 0.32939100 & -0.11826200 \\ \mathrm{C} & 2.80439200 & -0.14970400 & 0.23832300 \\ \mathrm{C} & 3.08320600 & -1.50098800 & 0.25716900 \\ \mathrm{C} & 2.08750300 & -2.40361600 & -0.10087500 \\ \mathrm{C} & 0.83479300 & -1.95041300 & -0.46869400 \\ \mathrm{H} & 4.06499600 & -1.85060100 & 0.54157100 \\ \mathrm{C} & 1.38554200 & 1.80803900 & -0.09711700 \\ \mathrm{O} & 2.30497400 & 2.57117000 & 0.04819900 \\ \mathrm{H} & 2.29090900 & -3.46610800 & -0.09967900 \\ \mathrm{H} & 3.55854900 & 0.57858400 & 0.50100700 \\ \mathrm{O} & 0.12394700 & 2.26025600 & -0.23356300 \\ \mathrm{H} & 0.18894800 & 3.22506300 & -0.20123100 \\ \mathrm{H} & 0.05624800 & -2.63742100 & -0.77142100 \\ \mathrm{O} & -0.68399300 & -0.18949300 & -0.87513900 \\ \mathrm{Si} & -2.05563300 & -0.15157200 & 0.09917700 \\ \mathrm{C} & -3.16866100 & 1.12356700 & -0.67149200 \\ \mathrm{H} & -3.38219400 & 0.86685200 & -1.70947200 \\ \mathrm{H} & -4.11690700 & 1.18941300 & -0.13669100 \\ \mathrm{H} & -2.69169900 & 2.10277500 & -0.65476900 \\ \mathrm{C} & -2.84621700 & -1.84593800 & 0.06080700 \\ \mathrm{H} & -2.20029900 & -2.59117900 & 0.52754800 \\ \mathrm{H} & -3.79282500 & -1.84169200 & 0.60386100 \\ \mathrm{H} & -3.04626500 & -2.16016300 & -0.96418400 \\ \mathrm{C} & -1.55479900 & 0.26508500 & 1.84828300 \\ \mathrm{H} & -2.42491300 & 0.23345300 & 2.50605300 \\ \mathrm{H} & -0.82826000 & -0.45736400 & 2.22484900 \\ \mathrm{H} & -1.11256800 & 1.25841800 & 1.90971100\end{array}$

$\begin{array}{lrrr}\text { 2-OEt } & & & \\ \text { C } & 0.18813700 & 0.52964800 & 0.00469100 \\ \text { C } & -0.96648700 & -0.27516100 & -0.00073500 \\ \text { C } & -2.21822200 & 0.33412900 & -0.00098900 \\ \text { C } & -2.35728800 & 1.70939600 & 0.02443500 \\ \text { C } & -1.21566700 & 2.49510900 & 0.04071400 \\ \text { C } & 0.04418100 & 1.91834000 & 0.02720100 \\ \text { H } & -3.33868100 & 2.16032100 & 0.03129400 \\ \text { C } & -0.98369000 & -1.76432500 & -0.01182400 \\ \text { O } & -1.97383400 & -2.40882000 & -0.24914300 \\ \text { H } & -1.29801400 & 3.57364700 & 0.06037300 \\ \text { H } & -3.08374900 & -0.31291500 & -0.01842800 \\ \text { O } & 0.17933000 & -2.35789700 & 0.29497200\end{array}$




$\begin{array}{rrrr}\mathrm{H} & 0.00489800 & -3.30875100 & 0.26586800 \\ \mathrm{H} & 0.91483600 & 2.55446900 & 0.03047400 \\ \mathrm{O} & 1.38954800 & -0.07410000 & -0.03339300 \\ \mathrm{C} & 2.56364800 & 0.72263300 & -0.03563700 \\ \mathrm{H} & 2.55829600 & 1.38303600 & -0.90749700 \\ \mathrm{H} & 2.58778400 & 1.34304900 & 0.86467000 \\ \mathrm{C} & 3.74281200 & -0.22002900 & -0.07589900 \\ \mathrm{H} & 3.70552500 & -0.83412800 & -0.97381300 \\ \mathrm{H} & 4.67330300 & 0.34595800 & -0.07628900 \\ \mathrm{H} & 3.73090600 & -0.87659800 & 0.79211200\end{array}$

$\begin{array}{lrrr}\text { 2-Cyclopropyl } & & \\ \mathrm{C} & -0.49946000 & 0.26854600 & -0.01457500 \\ \mathrm{C} & 0.88397100 & 0.03068100 & -0.01680600 \\ \mathrm{C} & 1.78672200 & 1.09628900 & 0.00873400 \\ \mathrm{C} & 1.34865900 & 2.40247100 & 0.08218100 \\ \mathrm{C} & -0.01735900 & 2.65047500 & 0.11461700 \\ \mathrm{C} & -0.91555200 & 1.60166300 & 0.05715200 \\ \mathrm{C} & 1.53726300 & -1.31050100 & -0.03881000 \\ \mathrm{O} & 2.62765100 & -1.51720200 & -0.50144900 \\ \mathrm{H} & 2.84230500 & 0.86638500 & -0.02194600 \\ \mathrm{O} & 0.83977300 & -2.29183900 & 0.56789600 \\ \mathrm{H} & 1.38976100 & -3.08584100 & 0.51539800 \\ \mathrm{H} & 2.05892800 & 3.21617900 & 0.11510000 \\ \mathrm{H} & -0.38534200 & 3.66587500 & 0.17547800 \\ \mathrm{H} & -1.97401000 & 1.82552100 & 0.06301900 \\ \mathrm{C} & -1.51994400 & -0.80641400 & -0.13087600 \\ \mathrm{C} & -2.80887200 & -0.70672800 & 0.64495300 \\ \mathrm{C} & -2.82513800 & -0.53931000 & -0.83612200 \\ \mathrm{H} & -1.13521300 & -1.80252200 & -0.24836600 \\ \mathrm{H} & -2.93999200 & 0.17320800 & 1.25790700 \\ \mathrm{H} & -3.20732100 & -1.61734000 & 1.06577900 \\ \mathrm{H} & -3.22816400 & -1.34147700 & -1.43565700 \\ \mathrm{H} & -2.98208000 & 0.44931100 & -1.24098900\end{array}$

$\begin{array}{lrrr}\text { 2-t-Bu } & & & \\ \text { C } & 0.91401800 & 0.31041000 & -0.02305700 \\ \text { C } & -0.10360600 & -0.65801500 & 0.00351900 \\ \text { C } & 0.31699800 & -1.98783400 & 0.07738900 \\ \text { C } & 1.65427800 & -2.35108300 & 0.11596600 \\ \text { C } & 2.63958000 & -1.38180000 & 0.05252900 \\ \text { C } & 2.25897500 & -0.05520500 & -0.02676800 \\ \text { H } & -0.41823100 & -2.77671800 & 0.09383700 \\ \text { H } & 1.91981500 & -3.39777400 & 0.17795700 \\ \text { H } & 3.68643300 & -1.65034800 & 0.05944800 \\ \text { H } & 3.00535300 & 0.72512200 & -0.09038700 \\ \text { C } & 0.72656000 & 1.79423500 & -0.04853700 \\ \text { O } & 1.17163000 & 2.52155100 & -0.89261000 \\ \text { O } & 0.09059200 & 2.27387300 & 1.03951900\end{array}$




$\begin{array}{lrrr}\mathrm{C} & -1.60512700 & -0.34195000 & -0.07261200 \\ \mathrm{C} & -2.13812400 & -0.01570800 & 1.33087000 \\ \mathrm{H} & -3.20977600 & 0.18694800 & 1.27780800 \\ \mathrm{H} & -1.98504700 & -0.86408300 & 1.99943300 \\ \mathrm{H} & -1.64048400 & 0.85140200 & 1.75641500 \\ \mathrm{C} & -1.89109500 & 0.82163600 & -1.03388700 \\ \mathrm{H} & -2.96706100 & 0.89682600 & -1.19383100 \\ \mathrm{H} & -1.56597600 & 1.78332700 & -0.64793500 \\ \mathrm{H} & -1.41475200 & 0.65700100 & -2.00116400 \\ \mathrm{C} & -2.39593800 & -1.54834700 & -0.60076500 \\ \mathrm{H} & -2.00590900 & -1.89521300 & -1.55863300 \\ \mathrm{H} & -2.39515300 & -2.38275500 & 0.09904300 \\ \mathrm{H} & -3.43512000 & -1.25240500 & -0.74287600 \\ \mathrm{H} & 0.06902500 & 3.23725800 & 0.94775100\end{array}$

$\begin{array}{lrrr}\text { 2-Me } & & & \\ \text { C } & -0.46739900 & 0.96842600 & 0.00006100 \\ \text { C } & 0.17338300 & -0.28203800 & 0.00005500 \\ \text { C } & -0.57638900 & -1.45884400 & 0.00003800 \\ \text { C } & -1.95728700 & -1.42787700 & -0.00001900 \\ \text { C } & -2.60229600 & -0.20000200 & -0.00005700 \\ \text { C } & -1.86205800 & 0.97058500 & 0.00000200 \\ \text { H } & -2.52321700 & -2.34856600 & -0.00003600 \\ \text { C } & 1.64922100 & -0.49426700 & -0.00000100 \\ \text { O } & 2.17493600 & -1.57656700 & 0.00001300 \\ \text { H } & -3.68296300 & -0.15093600 & -0.00011900 \\ \text { H } & -0.04156500 & -2.39774800 & 0.00006200 \\ \text { O } & 2.38539700 & 0.63367700 & -0.00007000 \\ \text { H } & 3.30964000 & 0.34899000 & -0.00009000 \\ \text { H } & -2.37627500 & 1.92269900 & 0.00000000 \\ \text { C } & 0.23833800 & 2.29844900 & 0.00004000 \\ \text { H } & 0.87844600 & 2.41020100 & 0.87289000 \\ \text { H } & 0.87640800 & 2.41114200 & -0.87420700 \\ \text { H } & -0.49621800 & 3.10074100 & 0.00124700\end{array}$

$\begin{array}{lrrr}2-i-P r & & & \\ \text { C } & 0.46692100 & -0.47458000 & -0.12804600 \\ \text { C } & -0.83612700 & 0.05477900 & -0.07512800 \\ \text { C } & -1.94154700 & -0.78451800 & 0.04536000 \\ \text { C } & -1.79366800 & -2.15901700 & 0.08545200 \\ \text { C } & -0.51921100 & -2.69575300 & 0.02240400 \\ \text { C } & 0.58573900 & -1.86183900 & -0.07397700 \\ \text { H } & -2.66102700 & -2.79871800 & 0.16600800 \\ \text { C } & -1.18821100 & 1.50553800 & -0.12296000 \\ \text { O } & -2.16815100 & 1.97296700 & 0.39384200 \\ \text { H } & -0.37682400 & -3.76769900 & 0.05375800 \\ \text { H } & -2.92113500 & -0.33137800 & 0.10265000 \\ \text { O } & -0.34756700 & 2.27384000 & -0.84302200 \\ \text { H } & -0.70543600 & 3.17183800 & -0.80935400\end{array}$




$\begin{array}{lrrr}\mathrm{H} & 1.56677500 & -2.31118200 & -0.10612900 \\ \mathrm{C} & 1.70956800 & 0.40092000 & -0.14860000 \\ \mathrm{H} & 1.57991000 & 1.14424200 & -0.93125500 \\ \mathrm{C} & 1.84629900 & 1.13704200 & 1.18992900 \\ \mathrm{H} & 1.98648000 & 0.41708800 & 1.99831100 \\ \mathrm{H} & 2.71050300 & 1.80166200 & 1.17110000 \\ \mathrm{H} & 0.96556600 & 1.73435200 & 1.42137900 \\ \mathrm{C} & 2.99880300 & -0.35844600 & -0.44674300 \\ \mathrm{H} & 3.25902800 & -1.04490000 & 0.36062000 \\ \mathrm{H} & 2.93176200 & -0.92689300 & -1.37432400 \\ \mathrm{H} & 3.81873900 & 0.35237300 & -0.54547000\end{array}$

$\begin{array}{lrrr}\text { 2-Cyclohexyl } & & \\ \mathrm{C} & -0.55212900 & -0.56357000 & -0.02154300 \\ \mathrm{C} & -1.67620300 & 0.27581400 & -0.06330900 \\ \mathrm{C} & -2.96441600 & -0.25163300 & 0.03343600 \\ \mathrm{C} & -3.16546400 & -1.60517000 & 0.22142400 \\ \mathrm{C} & -2.06321800 & -2.44386400 & 0.29778800 \\ \mathrm{C} & -0.78520400 & -1.92645300 & 0.16737100 \\ \mathrm{C} & -1.63858000 & 1.76104800 & -0.20634800 \\ \mathrm{O} & -2.50581300 & 2.40915600 & -0.72877500 \\ \mathrm{H} & -3.80095000 & 0.42939300 & -0.03589100 \\ \mathrm{O} & -0.56901000 & 2.35345100 & 0.36302700 \\ \mathrm{H} & -0.68288800 & 3.30492800 & 0.23048200 \\ \mathrm{H} & -4.16752600 & -2.00081600 & 0.30760500 \\ \mathrm{H} & -2.19727900 & -3.50666700 & 0.44865900 \\ \mathrm{H} & 0.05603700 & -2.60365900 & 0.20850300 \\ \mathrm{C} & 0.87031100 & -0.06970300 & -0.17802200 \\ \mathrm{C} & 1.53691500 & 0.12637200 & 1.19180900 \\ \mathrm{C} & 1.74414500 & -0.97521000 & -1.05489000 \\ \mathrm{H} & 0.84503900 & 0.90304900 & -0.66809400 \\ \mathrm{C} & 2.93646500 & 0.71589200 & 1.03836500 \\ \mathrm{H} & 1.59656600 & -0.84711100 & 1.69073600 \\ \mathrm{H} & 0.91489100 & 0.77043100 & 1.81284000 \\ \mathrm{C} & 3.13398100 & -0.36550900 & -1.22863400 \\ \mathrm{H} & 1.85695900 & -1.95817900 & -0.59060000 \\ \mathrm{H} & 1.26477000 & -1.13160400 & -2.02291300 \\ \mathrm{C} & 3.80685100 & -0.14353900 & 0.12434400 \\ \mathrm{H} & 3.40648000 & 0.82680500 & 2.01655500 \\ \mathrm{H} & 2.84944400 & 1.72068100 & 0.61271800 \\ \mathrm{H} & 3.74954200 & -1.01033700 & -1.85721900 \\ \mathrm{H} & 3.04478700 & 0.59290800 & -1.74928200 \\ \mathrm{H} & 4.78657300 & 0.31693200 & -0.00993800 \\ \mathrm{H} & 3.97541400 & -1.11445100 & 0.60106500\end{array}$

\section{2-Cyclobutyl}
$\mathrm{C}$
$0.36814200 \quad-0.79793200 \quad 0.08469800$
$\begin{array}{lllll}\text { C } & 0.84923600 & 0.51783700 & -0.00342000\end{array}$
$\begin{array}{lllll}\text { C } & 2.21917100 & 0.77089700 & -0.06603800\end{array}$ 


$\begin{array}{lrrr}\text { C } & 3.13663000 & -0.26278000 & -0.09715800 \\ \mathrm{C} & 2.67822600 & -1.56953000 & -0.03644800 \\ \mathrm{C} & 1.32021400 & -1.81926500 & 0.06851700 \\ \mathrm{C} & 0.01336300 & 1.75424200 & -0.02703800 \\ \mathrm{O} & 0.28445100 & 2.76274900 & 0.56678800 \\ \mathrm{H} & 2.54825500 & 1.80044000 & -0.09443000 \\ \mathrm{O} & -1.05597300 & 1.68324600 & -0.84379700 \\ \mathrm{H} & -1.49899300 & 2.54208100 & -0.79351800 \\ \mathrm{H} & 4.19432400 & -0.04983000 & -0.16059500 \\ \mathrm{H} & 3.37668600 & -2.39524600 & -0.05234900 \\ \mathrm{C} & -1.07196200 & -1.20694500 & 0.24856000 \\ \mathrm{C} & -2.07078900 & -0.40106200 & 1.11708000 \\ \mathrm{C} & -2.06671200 & -1.12402300 & -0.94372300 \\ \mathrm{H} & -1.06374200 & -2.23453300 & 0.61094700 \\ \mathrm{C} & -3.15431000 & -0.62934300 & 0.03725100 \\ \mathrm{H} & -1.80839300 & 0.64469300 & 1.24246300 \\ \mathrm{H} & -2.26584300 & -0.82059300 & 2.10167900 \\ \mathrm{H} & -1.78179900 & -0.37371900 & -1.67211600 \\ \mathrm{H} & -2.25743700 & -2.06596100 & -1.45355600 \\ \mathrm{H} & -3.71793300 & 0.24773100 & -0.27246600 \\ \mathrm{H} & -3.85673500 & -1.41323400 & 0.31369400 \\ \mathrm{H} & 0.97652900 & -2.84236600 & 0.15263000\end{array}$

\section{2-Cyclopentyl}

$\begin{array}{lrrr}\text { C } & 0.26886600 & 0.54407300 & -0.04829900 \\ \mathrm{C} & 1.41737400 & -0.26153200 & -0.05319500 \\ \mathrm{C} & 2.68927300 & 0.30642600 & 0.02858000 \\ \mathrm{C} & 2.84912300 & 1.67132400 & 0.16537300 \\ \mathrm{C} & 1.72157600 & 2.47892900 & 0.20718400 \\ \mathrm{C} & 0.46039700 & 1.91949500 & 0.09180800 \\ \mathrm{C} & 1.42048800 & -1.75150000 & -0.13098900 \\ \mathrm{O} & 2.30151200 & -2.39879600 & -0.63076200 \\ \mathrm{H} & 3.54567400 & -0.35202300 & -0.00964600 \\ \mathrm{O} & 0.37139900 & -2.34450200 & 0.47415700 \\ \mathrm{H} & 0.50916500 & -3.29781300 & 0.38457200 \\ \mathrm{H} & 3.83840300 & 2.10009200 & 0.23980800 \\ \mathrm{H} & 1.82332300 & 3.55014100 & 0.31848200 \\ \mathrm{H} & -0.40055500 & 2.57269900 & 0.10573500 \\ \mathrm{C} & -1.13228100 & 0.00223600 & -0.19932900 \\ \mathrm{C} & -1.89830400 & -0.12097200 & 1.12825700 \\ \mathrm{C} & -2.10588700 & 0.82421100 & -1.05188400 \\ \mathrm{H} & -1.08410800 & -0.98796100 & -0.64984200 \\ \mathrm{C} & -3.37488200 & -0.29049100 & 0.71357200 \\ \mathrm{H} & -1.76230500 & 0.80466300 & 1.69218100 \\ \mathrm{H} & -1.52220100 & -0.93779400 & 1.74033500 \\ \mathrm{C} & -3.44603400 & 0.13769300 & -0.77339900 \\ \mathrm{H} & -1.83628900 & 0.84005000 & -2.10727500 \\ \mathrm{H} & -2.14617300 & 1.85672500 & -0.70160900 \\ \mathrm{H} & -3.70095500 & -1.32227800 & 0.83403200\end{array}$




$\begin{array}{lrrr}H & -4.02632400 & 0.32083600 & 1.33582700 \\ H & -3.54166000 & -0.74197100 & -1.41081000 \\ H & -4.29754000 & 0.78166700 & -0.98502800\end{array}$

$\begin{array}{lrrr}\text { 2-Bn } & & & \\ \mathrm{C} & -1.14393000 & -0.64155100 & -0.66216500 \\ \mathrm{C} & -1.53446800 & 0.55625200 & -0.04480800 \\ \mathrm{C} & -2.73410600 & 0.63040400 & 0.65794500 \\ \mathrm{C} & -3.57893400 & -0.46256600 & 0.74069700 \\ \mathrm{C} & -3.20852500 & -1.64982800 & 0.12950100 \\ \mathrm{C} & -2.00232700 & -1.73175800 & -0.55087400 \\ \mathrm{H} & -4.51257500 & -0.38851600 & 1.28011100 \\ \mathrm{C} & -0.73252100 & 1.81446800 & -0.05123400 \\ \mathrm{O} & -0.80522700 & 2.66026100 & 0.79923500 \\ \mathrm{H} & -3.85142200 & -2.51767900 & 0.18806900 \\ \mathrm{H} & -2.98746500 & 1.56620000 & 1.13611800 \\ \mathrm{O} & 0.06117500 & 1.95873900 & -1.12752300 \\ \mathrm{H} & 0.54480700 & 2.78723500 & -1.00503000 \\ \mathrm{H} & -1.71097300 & -2.66975700 & -1.00565500 \\ \mathrm{C} & 0.17422800 & -0.83097200 & -1.37474300 \\ \mathrm{H} & 0.24357100 & -0.15484200 & -2.22485700 \\ \mathrm{H} & 0.19120000 & -1.84612400 & -1.77670800 \\ \mathrm{C} & 1.39624800 & -0.64040200 & -0.50023800 \\ \mathrm{C} & 2.58200600 & -0.17393400 & -1.06134100 \\ \mathrm{C} & 1.37905900 & -0.95276800 & 0.85508100 \\ \mathrm{C} & 3.72532500 & -0.02840300 & -0.29118000 \\ \mathrm{H} & 2.60285300 & 0.08519400 & -2.11300700 \\ \mathrm{C} & 2.52193000 & -0.80588300 & 1.63071800 \\ \mathrm{H} & 0.46360500 & -1.30714100 & 1.31216500 \\ \mathrm{C} & 3.69869200 & -0.34454300 & 1.06098000 \\ \mathrm{H} & 4.63648700 & 0.33835600 & -0.74443800 \\ \mathrm{H} & 2.48893800 & -1.04972400 & 2.68393800 \\ \mathrm{H} & 4.58731600 & -0.22629300 & 1.66558100\end{array}$

$\begin{array}{lrrr}\text { 2-SiMe } & & & \\ \mathrm{C} & & & \\ \mathrm{C} & 0.19955800 & -0.60452600 & 0.03602400 \\ \mathrm{C} & 1.27953600 & 0.29658900 & 0.01563300 \\ \mathrm{C} & 2.60023000 & -0.14467800 & -0.00456300 \\ \mathrm{C} & 2.89129800 & -1.49658800 & 0.02416500 \\ \mathrm{C} & 1.84904400 & -2.40844300 & 0.05824500 \\ \mathrm{C} & 1.12567100 & 1.77765300 & 0.01398200 \\ \mathrm{O} & 1.98301700 & 2.55937000 & -0.29915500 \\ \mathrm{H} & 3.38802300 & 0.59481200 & -0.03749100 \\ \mathrm{O} & -0.08572100 & 2.19288600 & 0.43348000 \\ \mathrm{H} & -0.08658300 & 3.15804000 & 0.37369300 \\ \mathrm{H} & 3.91867500 & -1.83297900 & 0.01864000 \\ \mathrm{H} & 2.05508000 & -3.47036400 & 0.07975600 \\ \mathrm{C} & 0.53394000 & -1.96282200 & 0.05300500 \\ \mathrm{H} & -0.25247200 & -2.70487300 & 0.05532100\end{array}$




$\begin{array}{lrrr}\mathrm{Si} & -1.66629000 & -0.19737100 & -0.03665100 \\ \mathrm{C} & -2.32027400 & 0.34482200 & 1.63569500 \\ \mathrm{H} & -3.39973500 & 0.49638500 & 1.57572300 \\ \mathrm{H} & -2.13547600 & -0.43140500 & 2.37964300 \\ \mathrm{H} & -1.85884000 & 1.26729100 & 1.97920000 \\ \mathrm{C} & -2.06186000 & 1.03176600 & -1.40957100 \\ \mathrm{H} & -2.36509800 & 1.99503400 & -1.00254900 \\ \mathrm{H} & -1.19956300 & 1.19976700 & -2.05526500 \\ \mathrm{H} & -2.87474900 & 0.65261600 & -2.02960300 \\ \mathrm{C} & -2.55611100 & -1.80194700 & -0.46222300 \\ \mathrm{H} & -2.19130000 & -2.24029200 & -1.39215000 \\ \mathrm{H} & -2.47783600 & -2.55103700 & 0.32621500 \\ \mathrm{H} & -3.61661900 & -1.57880700 & -0.59496900\end{array}$

\section{2-OPh}

C

C

C

C

C

C

$\mathrm{H}$

C

$\mathrm{O}$

$\mathrm{H}$

$\mathrm{H}$

$\mathrm{O}$

$\mathrm{H}$

$\mathrm{H}$

O

C

C

C

C

$\mathrm{H}$

C

$\mathrm{H}$

C

$\mathrm{H}$

$\mathrm{H}$

H

$\begin{array}{rrr}1.04752800 & 0.72287900 & -0.50047000 \\ 1.51265200 & -0.53000600 & -0.09151900 \\ 2.77609300 & -0.62047400 & 0.48845900 \\ 3.58101300 & 0.49565400 & 0.62753700 \\ 3.11582300 & 1.73065700 & 0.19603600 \\ 1.85010500 & 1.84464500 & -0.35506400 \\ 4.56353200 & 0.40382900 & 1.06750200 \\ 0.75468300 & -1.80633100 & -0.22205200 \\ 0.95060900 & -2.76776200 & 0.47221000 \\ 3.73436300 & 2.61171200 & 0.29872800 \\ 3.10993200 & -1.59475600 & 0.81718700 \\ -0.14122600 & -1.80939300 & -1.21908900 \\ -0.57974200 & -2.67124700 & -1.19915900 \\ 1.45671000 & 2.79782400 & -0.67858600 \\ -0.18001500 & 0.91186200 & -1.07661400 \\ -1.31800100 & 0.65092200 & -0.35286900 \\ -2.49532300 & 0.56201000 & -1.08400500 \\ -1.32703800 & 0.51310100 & 1.02733800 \\ -3.68959400 & 0.32973900 & -0.42563800 \\ -2.44867300 & 0.67120100 & -2.15821500 \\ -2.53482900 & 0.27473600 & 1.67304500 \\ -0.41052600 & 0.59217800 & 1.59470200 \\ -3.71669400 & 0.18101300 & 0.95681100 \\ -4.60566500 & 0.25943100 & -0.99622900 \\ -2.54260700 & 0.16545200 & 2.74890200 \\ -4.65077800 & -0.00454100 & 1.46744600\end{array}$

\section{2-Ph}

$\begin{array}{lrrr}\text { C } & -0.51074000 & -0.67185100 & -0.02298300 \\ \text { C } & -1.42938700 & 0.38363400 & 0.03419400 \\ \text { C } & -2.79581400 & 0.13686200 & -0.05996600 \\ C & -3.27248900 & -1.15817500 & -0.16282500 \\ C & -2.37331000 & -2.21484400 & -0.18561800 \\ C & -1.01101400 & -1.96934500 & -0.12737800\end{array}$




$\begin{array}{lrrr}\text { H } & -4.33593600 & -1.34159000 & -0.22327100 \\ \mathrm{C} & -1.04592700 & 1.81114600 & 0.24019500 \\ \mathrm{O} & -1.60622700 & 2.74064600 & -0.27668000 \\ \mathrm{H} & -2.73163800 & -3.23188900 & -0.26872100 \\ \mathrm{H} & -3.47359000 & 0.97896900 & -0.03469800 \\ \mathrm{O} & -0.04688000 & 1.97316000 & 1.12240700 \\ \mathrm{H} & 0.12193800 & 2.92307300 & 1.18854400 \\ \mathrm{H} & -0.31019000 & -2.79088700 & -0.19533600 \\ \mathrm{C} & 0.95715900 & -0.46748000 & -0.06330900 \\ \mathrm{C} & 1.79767200 & -1.20556200 & 0.76526100 \\ \mathrm{C} & 1.52073500 & 0.43156100 & -0.96669300 \\ \mathrm{C} & 3.17335400 & -1.03794500 & 0.70326200 \\ \mathrm{H} & 1.36680800 & -1.89862900 & 1.47628800 \\ \mathrm{C} & 2.89460700 & 0.59580800 & -1.03327500 \\ \mathrm{H} & 0.87527100 & 0.99576000 & -1.62911500 \\ \mathrm{C} & 3.72545200 & -0.13714800 & -0.19591300 \\ \mathrm{H} & 3.81400800 & -1.60938200 & 1.36099200 \\ \mathrm{H} & 3.31851800 & 1.29240900 & -1.74366500 \\ \mathrm{H} & 4.79788200 & -0.00824300 & -0.24656800 \\ & & & \\ 2-\mathrm{F} & & & \\ \mathrm{C} & -0.48660800 & 0.94299000 & -0.00001500 \\ \mathrm{C} & 0.20610900 & -0.26365400 & 0.00000600 \\ \mathrm{C} & -0.54129100 & -1.44235700 & 0.00000600 \\ \mathrm{C} & -1.92212800 & -1.41725300 & -0.00001300 \\ \mathrm{C} & -2.58583000 & -0.19568000 & -0.00003400 \\ \mathrm{C} & -1.86933200 & 0.98820200 & -0.00003500 \\ \mathrm{H} & -2.48095700 & -2.34168200 & -0.00001300 \\ \mathrm{C} & 1.68758200 & -0.40907000 & 0.00002800 \\ \mathrm{O} & 2.24521900 & -1.47437500 & 0.00005400 \\ \mathrm{H} & -3.66664000 & -0.16340300 & -0.00005000 \\ \mathrm{H} & 0.00355700 & -2.37580400 & 0.00002300 \\ \mathrm{O} & 2.35833400 & 0.75024400 & 0.00001400 \\ \mathrm{H} & 3.29868500 & 0.52396100 & 0.00003100 \\ \mathrm{~F} & 0.16013200 & 2.10864300 & -0.00001600 \\ \mathrm{H} & -2.35527000 & 1.95312600 & -0.00005000\end{array}$

$\begin{array}{lrrr}\mathbf{2 - N} \mathbf{N}_{3} & & & \\ \mathrm{C} & 0.38689000 & -0.84993300 & -0.17420000 \\ \mathrm{C} & 0.33766200 & 0.54043500 & -0.00464100 \\ \mathrm{C} & 1.52658700 & 1.23759400 & 0.21593000 \\ \mathrm{C} & 2.74796100 & 0.59439900 & 0.20952200 \\ \mathrm{C} & 2.79253600 & -0.77640200 & -0.01775000 \\ \mathrm{C} & 1.62431000 & -1.49027800 & -0.20044600 \\ \mathrm{C} & -0.88084500 & 1.38862300 & -0.11609100 \\ \mathrm{O} & -1.00650700 & 2.46198000 & 0.40686700 \\ \mathrm{H} & 1.45902400 & 2.30562500 & 0.36870800 \\ \mathrm{O} & -1.82037900 & 0.87190600 & -0.92840300 \\ \mathrm{H} & -2.55896300 & 1.49659300 & -0.94042100\end{array}$




$\begin{array}{lrrr}H & 1.63784900 & -2.56088600 & -0.34546100 \\ H & 3.65779300 & 1.15496900 & 0.36779000 \\ H & 3.74136900 & -1.29467000 & -0.03727600 \\ \mathrm{~N} & -0.72387800 & -1.71536400 & -0.33215500 \\ \mathrm{~N} & -1.75762400 & -1.46065100 & 0.28273100 \\ \mathrm{~N} & -2.73743900 & -1.34389300 & 0.80442500\end{array}$

\section{2-Si(OMe)}

$\begin{array}{lrrr}\text { C } & -0.81858400 & -0.63428400 & 0.07588500 \\ \text { C } & -1.88394700 & 0.27459000 & -0.02026300 \\ \text { C } & -3.20555200 & -0.15447600 & -0.05612000 \\ \text { C } & -3.50391900 & -1.50352600 & 0.02932600 \\ \text { C } & -2.47116800 & -2.42094200 & 0.13605200 \\ \text { C } & -1.68614300 & 1.74607500 & -0.09339200 \\ \text { O } & -2.49895600 & 2.53809200 & -0.48526400 \\ \text { H } & -3.98602200 & 0.58798400 & -0.14719700 \\ \text { O } & -0.47801500 & 2.12885600 & 0.36187100 \\ \text { H } & -0.39950800 & 3.07972000 & 0.20762400 \\ \text { H } & -4.53306300 & -1.83442600 & 0.01108200 \\ \text { H } & -2.68894800 & -3.47853500 & 0.20141500 \\ \mathrm{C} & -1.15097100 & -1.98994800 & 0.14840200 \\ \text { H } & -0.35817500 & -2.72182200 & 0.20526400 \\ \mathrm{Si} & 1.01196200 & -0.23634700 & 0.02021900 \\ \mathrm{O} & 1.35312200 & 0.71053000 & -1.27693500 \\ \mathrm{O} & 1.72369400 & -1.70480500 & -0.16154700 \\ \mathrm{O} & 1.67340600 & 0.38473900 & 1.38015900 \\ \mathrm{C} & 3.03403400 & -2.04345000 & 0.24272900 \\ \mathrm{H} & 3.30137600 & -1.54364200 & 1.17474900 \\ \mathrm{H} & 3.08001000 & -3.12103700 & 0.39203800 \\ \mathrm{H} & 3.75888400 & -1.76954500 & -0.52740900 \\ \mathrm{C} & 2.09252300 & 1.70714800 & 1.62525900 \\ \mathrm{H} & 3.06313100 & 1.67896400 & 2.12069600 \\ \mathrm{H} & 2.18606400 & 2.27487600 & 0.69656400 \\ \mathrm{H} & 1.37850000 & 2.21202400 & 2.27565200 \\ \mathrm{C} & 2.60163000 & 0.62660000 & -1.93562200 \\ \mathrm{H} & 2.74287900 & -0.35959400 & -2.38277900 \\ \mathrm{H} & 2.62318800 & 1.37715600 & -2.72315300 \\ \mathrm{H} & 3.43079700 & 0.82073000 & -1.24742300\end{array}$

$\begin{array}{lrrr}2-\mathrm{Cl} & & & \\ \mathrm{C} & 0.45203900 & 0.73941800 & -0.01630900 \\ \mathrm{C} & -0.09565500 & -0.54397500 & 0.00337400 \\ \mathrm{C} & 0.77153900 & -1.63672600 & 0.03462700 \\ \mathrm{C} & 2.14184300 & -1.46766000 & 0.07854800 \\ \mathrm{C} & 2.67014400 & -0.18409900 & 0.07335000 \\ \mathrm{C} & 1.82952700 & 0.91379300 & 0.01669200 \\ \mathrm{H} & 2.79328300 & -2.32891800 & 0.11332300 \\ \mathrm{C} & -1.54862200 & -0.89032500 & -0.01539700\end{array}$




$\begin{array}{lrrr}\mathrm{O} & -1.96616800 & -1.93509700 & -0.43791200 \\ \mathrm{H} & 3.74031500 & -0.03257500 & 0.10433000 \\ \mathrm{H} & 0.33171500 & -2.62391700 & 0.02632600 \\ \mathrm{O} & -2.34921000 & 0.03403700 & 0.52848500 \\ \mathrm{H} & -3.25146500 & -0.31037500 & 0.47226100 \\ \mathrm{H} & 2.22844600 & 1.91717300 & -0.00986600 \\ \mathrm{Cl} & -0.50848000 & 2.17673800 & -0.14589800\end{array}$

$\begin{array}{lrrr}\text { 2-Br } & & & \\ \mathrm{C} & 0.04103700 & -0.64122500 & -0.02445000 \\ \mathrm{C} & 0.86601300 & 0.48336900 & -0.01168200 \\ \mathrm{C} & 2.24877700 & 0.29657100 & -0.01053300 \\ \mathrm{C} & 2.80336700 & -0.96802800 & -0.05802200 \\ \mathrm{C} & 1.96941900 & -2.07655400 & -0.08651600 \\ \mathrm{C} & 0.59440400 & -1.91469000 & -0.05843000 \\ \mathrm{H} & 3.87707500 & -1.08862500 & -0.06881600 \\ \mathrm{C} & 0.42488500 & 1.91069000 & 0.01162100 \\ \mathrm{O} & 1.06455700 & 2.78570700 & 0.52975200 \\ \mathrm{H} & 2.38549900 & -3.07405300 & -0.12006700 \\ \mathrm{H} & 2.87358400 & 1.17783000 & 0.02555600 \\ \mathrm{O} & -0.71453500 & 2.14692000 & -0.64934900 \\ \mathrm{H} & -0.89123200 & 3.09526200 & -0.57623700 \\ \mathrm{H} & -0.05954700 & -2.77401000 & -0.05486300 \\ \mathrm{Br} & -1.84779900 & -0.55252000 & 0.09083600\end{array}$

$\begin{array}{crrr}\mathbf{2 - F} \mathbf{F}_{\mathbf{F}} \mathbf{P h} & & & \\ \mathrm{C} & 1.30000000 & 0.59850000 & -0.24560000 \\ \mathrm{C} & 2.27140000 & -0.29720000 & 0.21950000 \\ \mathrm{C} & 3.62070000 & -0.01550000 & 0.02680000 \\ \mathrm{C} & 4.02130000 & 1.15460000 & -0.59240000 \\ \mathrm{C} & 3.06420000 & 2.05400000 & -1.03720000 \\ \mathrm{C} & 1.71890000 & 1.77110000 & -0.87100000 \\ \mathrm{H} & 5.07330000 & 1.36330000 & -0.72640000 \\ \mathrm{C} & 1.98910000 & -1.56540000 & 0.95590000 \\ \mathrm{O} & 2.75930000 & -2.48320000 & 1.02130000 \\ \mathrm{H} & 3.36140000 & 2.97260000 & -1.52370000 \\ \mathrm{H} & 4.34490000 & -0.73420000 & 0.38300000 \\ \mathrm{O} & 0.80730000 & -1.57740000 & 1.59790000 \\ \mathrm{H} & 0.74320000 & -2.43330000 & 2.04440000 \\ \mathrm{H} & 0.97360000 & 2.46130000 & -1.24150000 \\ \mathrm{C} & -0.16050000 & 0.34760000 & -0.17180000 \\ \mathrm{C} & -1.02940000 & 1.28440000 & 0.36890000 \\ \mathrm{C} & -0.72960000 & -0.81050000 & -0.68370000 \\ \mathrm{C} & -2.39840000 & 1.08250000 & 0.40920000 \\ \mathrm{C} & -2.09140000 & -1.03640000 & -0.66030000 \\ \mathrm{C} & -2.93130000 & -0.08270000 & -0.10950000 \\ \mathrm{~F} & 0.05020000 & -1.75200000 & -1.21010000 \\ \mathrm{~F} & -2.60040000 & -2.15580000 & -1.15810000 \\ \mathrm{~F} & -4.23890000 & -0.28720000 & -0.08080000\end{array}$




$\begin{array}{llll}F & -3.19820000 & 1.99660000 & 0.94260000 \\ F & -0.55700000 & 2.41450000 & 0.89070000\end{array}$

$\begin{array}{lrrr}2-O A C & & & \\ \text { C } & 0.20453200 & 0.65104000 & -0.42335400 \\ \text { C } & 0.86978700 & -0.52849900 & -0.08174800 \\ \text { C } & 2.22037800 & -0.46204600 & 0.25792300 \\ \text { C } & 2.89328500 & 0.74509200 & 0.27736800 \\ \text { C } & 2.21946200 & 1.91032300 & -0.06620600 \\ \text { C } & 0.88266100 & 1.86114900 & -0.42319500 \\ \text { H } & 3.93827700 & 0.77837700 & 0.54972200 \\ \text { C } & 0.25219800 & -1.88492300 & -0.06934800 \\ \text { O } & 0.88656500 & -2.90390500 & -0.09714600 \\ \text { H } & 2.73762400 & 2.85939000 & -0.06586200 \\ \text { H } & 2.72306200 & -1.38698100 & 0.50344100 \\ \text { O } & -1.08778500 & -1.87509100 & 0.01489300 \\ \text { H } & -1.38381400 & -2.79597300 & 0.00287700 \\ \text { O } & -1.09591000 & 0.65016800 & -0.86868300 \\ \text { C } & -2.15816900 & 0.68924400 & -0.00083000 \\ \text { C } & -1.83226400 & 0.89187900 & 1.45169800 \\ H & -1.23072000 & 0.06362100 & 1.82205200 \\ \text { H } & -1.25708300 & 1.80739200 & 1.58707600 \\ \text { H } & -2.76434400 & 0.95008200 & 2.00232200 \\ \text { O } & -3.25515300 & 0.59560200 & -0.45093800 \\ \text { H } & 0.34404100 & 2.75034900 & -0.72048600\end{array}$

$\begin{array}{lrrr}2-\mathrm{CHO} & & & \\ \mathrm{C} & 0.58361300 & 0.55852800 & -0.06619600 \\ \mathrm{C} & -0.33616700 & -0.49703800 & -0.03590000 \\ \mathrm{C} & 0.11977400 & -1.80987200 & 0.02916700 \\ \mathrm{C} & 1.47624400 & -2.08092200 & 0.08675500 \\ \mathrm{C} & 2.39112100 & -1.03654100 & 0.07638400 \\ \mathrm{C} & 1.94421700 & 0.27095900 & -0.00542700 \\ \mathrm{H} & 1.81835400 & -3.10490700 & 0.14260500 \\ \mathrm{C} & -1.81996900 & -0.33604900 & -0.01493900 \\ \mathrm{O} & -2.59420300 & -1.18484900 & -0.36133700 \\ \mathrm{H} & 3.45158100 & -1.24252700 & 0.12195700 \\ \mathrm{H} & -0.60980500 & -2.60702600 & 0.04546400 \\ \mathrm{O} & -2.22747100 & 0.84578500 & 0.48374700 \\ \mathrm{H} & -3.19499700 & 0.83823800 & 0.47677100 \\ \mathrm{H} & 2.63867700 & 1.09901100 & -0.03707600 \\ \mathrm{C} & 0.21487800 & 1.99657000 & -0.25417000 \\ \mathrm{H} & -0.77107300 & 2.20645200 & -0.68685600 \\ \mathrm{O} & 0.97479900 & 2.89118200 & 0.00797600\end{array}$

\section{2-COPh}

$\begin{array}{lrrr}\text { C } & -1.16826800 & -0.74941100 & 0.15066000 \\ \text { C } & -1.58061100 & 0.58558400 & 0.16293100 \\ \text { C } & -2.82301700 & 0.93693100 & -0.35244400\end{array}$




\begin{tabular}{|c|c|c|c|}
\hline C & -3.66873900 & -0.03348200 & -0.86389400 \\
\hline C & -3.27351200 & -1.36344400 & -0.85676500 \\
\hline C & -2.03102200 & -1.71645000 & -0.35223300 \\
\hline C & -0.76777100 & 1.68743900 & 0.75154200 \\
\hline $\mathrm{O}$ & -0.82922300 & 2.83538200 & 0.40532800 \\
\hline $\mathrm{H}$ & -3.11261900 & 1.97836200 & -0.33240100 \\
\hline $\mathrm{O}$ & 0.02195300 & 1.27004200 & 1.75435800 \\
\hline $\mathrm{H}$ & 0.53803900 & 2.03077300 & 2.05500500 \\
\hline $\mathrm{H}$ & -4.63537100 & 0.24633800 & -1.25854400 \\
\hline $\mathrm{H}$ & -3.93134300 & -2.12790900 & -1.24677000 \\
\hline $\mathrm{H}$ & -1.71072100 & -2.74929900 & -0.34663200 \\
\hline C & 0.18321700 & -1.23567200 & 0.60602600 \\
\hline $\mathrm{O}$ & 0.25290900 & -2.15616900 & 1.38438700 \\
\hline C & 1.41150600 & -0.63313300 & 0.00288600 \\
\hline C & 1.36653800 & 0.16055400 & -1.13937700 \\
\hline C & 2.63719700 & -0.90860900 & 0.60553100 \\
\hline C & 2.53896000 & 0.68120700 & -1.67033900 \\
\hline $\mathrm{H}$ & 0.42195800 & 0.36668800 & -1.62475800 \\
\hline C & 3.80326600 & -0.38201800 & 0.07997600 \\
\hline $\mathrm{H}$ & 2.64981100 & -1.53546600 & 1.48637800 \\
\hline C & 3.75479100 & 0.41502200 & -1.05925900 \\
\hline $\mathrm{H}$ & 2.50042300 & 1.29441500 & -2.55979300 \\
\hline $\mathrm{H}$ & 4.75250200 & -0.59060600 & 0.55403100 \\
\hline $\mathrm{H}$ & 4.66700300 & 0.82554700 & -1.47055700 \\
\hline
\end{tabular}

$\begin{array}{lrrr}2-\mathrm{CO}_{2} \mathrm{Ph} & & & \\ \mathrm{C} & -1.61080000 & -0.71460000 & 0.13770000 \\ \mathrm{C} & -2.12190000 & 0.57110000 & -0.04500000 \\ \mathrm{C} & -3.46620000 & 0.74000000 & -0.34860000 \\ \mathrm{C} & -4.30490000 & -0.35990000 & -0.44410000 \\ \mathrm{C} & -3.80270000 & -1.63510000 & -0.22960000 \\ \mathrm{C} & -2.45800000 & -1.81100000 & 0.05990000 \\ \mathrm{C} & -1.31490000 & 1.81240000 & 0.14140000 \\ \mathrm{O} & -1.47340000 & 2.82280000 & -0.48430000 \\ \mathrm{H} & -3.84160000 & 1.74330000 & -0.49410000 \\ \mathrm{O} & -0.43150000 & 1.71000000 & 1.15110000 \\ \mathrm{H} & 0.05640000 & 2.54440000 & 1.19160000 \\ \mathrm{H} & -5.35120000 & -0.21970000 & -0.67660000 \\ \mathrm{H} & -4.45540000 & -2.49440000 & -0.29470000 \\ \mathrm{C} & -0.16120000 & -0.99110000 & 0.36070000 \\ \mathrm{O} & 0.60010000 & -0.24960000 & -0.47610000 \\ \mathrm{O} & 0.26270000 & -1.80750000 & 1.12340000 \\ \mathrm{H} & -2.04560000 & -2.79820000 & 0.21590000 \\ \mathrm{C} & 1.97810000 & -0.21590000 & -0.30560000 \\ \mathrm{C} & 2.75070000 & -0.33030000 & -1.44680000 \\ \mathrm{C} & 2.54700000 & 0.00750000 & 0.93770000 \\ \mathrm{C} & 4.12950000 & -0.22110000 & -1.34170000 \\ \mathrm{H} & 2.26510000 & -0.49610000 & -2.39800000 \\ \mathrm{C} & 3.92680000 & 0.11220000 & 1.02810000\end{array}$




$\begin{array}{lrrr}\mathrm{H} & 1.91920000 & 0.08570000 & 1.81290000 \\ \mathrm{C} & 4.72030000 & -0.00170000 & -0.10570000 \\ \mathrm{H} & 4.74070000 & -0.30810000 & -2.22930000 \\ \mathrm{H} & 4.38310000 & 0.28210000 & 1.99350000 \\ \mathrm{H} & 5.79500000 & 0.08060000 & -0.02510000\end{array}$

$\begin{array}{lrrr}2-\mathrm{CO}_{2} \mathrm{Me} & & & \\ \mathrm{C} & -0.33211600 & -0.71447000 & -0.08044400 \\ \mathrm{C} & -0.83879000 & 0.58306800 & 0.00188800 \\ \mathrm{C} & -2.20389100 & 0.78063300 & 0.16311500 \\ \mathrm{C} & -3.06639800 & -0.30364300 & 0.21358900 \\ \mathrm{C} & -2.56612600 & -1.59200500 & 0.09674500 \\ \mathrm{C} & -1.20200700 & -1.79539000 & -0.04813200 \\ \mathrm{C} & -0.00071400 & 1.80749300 & -0.15431600 \\ \mathrm{O} & -0.19600100 & 2.84063400 & 0.42288600 \\ \mathrm{H} & -2.57557400 & 1.79343300 & 0.23315300 \\ \mathrm{O} & 0.95897500 & 1.66408700 & -1.08580600 \\ \mathrm{H} & 1.43858600 & 2.50248500 & -1.13096900 \\ \mathrm{H} & -4.12828300 & -0.14108000 & 0.33426800 \\ \mathrm{H} & -3.23599300 & -2.44006700 & 0.12601200 \\ \mathrm{H} & -0.79239700 & -2.79298700 & -0.12559500 \\ \mathrm{C} & 1.12898700 & -1.02098400 & -0.13290700 \\ \mathrm{O} & 1.61488600 & -1.88166300 & -0.81421500 \\ \mathrm{O} & 1.82306600 & -0.26826400 & 0.72641300 \\ \mathrm{C} & 3.23691600 & -0.45678300 & 0.69134900 \\ \mathrm{H} & 3.48694900 & -1.48894300 & 0.92714200 \\ \mathrm{H} & 3.61843600 & -0.21615500 & -0.29968800 \\ \mathrm{H} & 3.64570100 & 0.21745600 & 1.43614400\end{array}$

$\begin{array}{lrrr}2-\mathrm{CO}_{2} \mathrm{H} & & & \\ \mathrm{C} & -0.28550900 & -0.69793300 & 0.00701200 \\ \mathrm{C} & -0.28662300 & 0.69789900 & -0.00641700 \\ \mathrm{C} & -1.49304600 & 1.38419700 & -0.03687700 \\ \mathrm{C} & -2.69392300 & 0.69107500 & -0.02451500 \\ \mathrm{C} & -2.69284900 & -0.69477900 & 0.02423500 \\ \mathrm{C} & -1.49090600 & -1.38606900 & 0.03698900 \\ \mathrm{C} & 0.94930800 & 1.52856100 & 0.09498700 \\ \mathrm{O} & 1.11009400 & 2.57498200 & -0.46799600 \\ \mathrm{H} & -1.47302100 & 2.46495600 & -0.05585100 \\ \mathrm{O} & 1.84006300 & 1.01902400 & 0.96211700 \\ \mathrm{H} & 2.60395500 & 1.61192500 & 0.97030100 \\ \mathrm{H} & -3.62836400 & 1.23424300 & -0.04375600 \\ \mathrm{H} & -3.62644700 & -1.23940900 & 0.04317400 \\ \mathrm{H} & -1.46929900 & -2.46680100 & 0.05598200 \\ \mathrm{C} & 0.95150700 & -1.52709000 & -0.09470300 \\ \mathrm{O} & 1.11436900 & -2.57248500 & 0.46953000 \\ \mathrm{O} & 1.84051300 & -1.01780300 & -0.96393000 \\ \mathrm{H} & 2.60511000 & -1.60982800 & -0.97188900\end{array}$




$\begin{array}{lrrr}2-\mathrm{CCl}_{3} & & & \\ \mathrm{C} & -0.26527800 & 0.67771800 & 0.00497700 \\ \mathrm{C} & -1.40436100 & -0.13895000 & -0.03035800 \\ \mathrm{C} & -2.66850200 & 0.43978900 & -0.04349000 \\ \mathrm{C} & -2.82986800 & 1.81198500 & 0.03826200 \\ \mathrm{C} & -1.70925900 & 2.61791300 & 0.12354700 \\ \mathrm{C} & -0.44331100 & 2.05413500 & 0.09399900 \\ \mathrm{H} & -3.82112400 & 2.24261400 & 0.03522600 \\ \mathrm{C} & -1.43458100 & -1.63838500 & -0.02909600 \\ \mathrm{O} & -2.07381900 & -2.29256800 & -0.80356900 \\ \mathrm{H} & -1.80921500 & 3.69184600 & 0.19462600 \\ \mathrm{H} & -3.52732300 & -0.21309800 & -0.11347900 \\ \mathrm{O} & -0.76339300 & -2.17075000 & 1.00120500 \\ \mathrm{H} & -0.83975200 & -3.13281400 & 0.92919200 \\ \mathrm{H} & 0.41825200 & 2.70117900 & 0.12886700 \\ \mathrm{C} & 1.15409500 & 0.12001400 & -0.04367900 \\ \mathrm{Cl} & 1.68534100 & -0.33958500 & 1.59561100 \\ \mathrm{Cl} & 1.27377000 & -1.28577600 & -1.13345400 \\ \mathrm{Cl} & 2.32813900 & 1.31662700 & -0.66453900\end{array}$

$\begin{array}{lrrr}\text { 2-COMe } & & & \\ \text { C } & -0.16189300 & 0.71570000 & -0.04254700 \\ \text { C } & -0.42519900 & -0.65658900 & -0.00364300 \\ \text { C } & -1.73684200 & -1.11366100 & 0.06631800 \\ \text { C } & -2.79138400 & -0.21668000 & 0.08681200 \\ \text { C } & -2.53591000 & 1.14561800 & 0.02210100 \\ \text { C } & -1.22948200 & 1.60610000 & -0.04474300 \\ \text { C } & 0.63193600 & -1.69832500 & -0.11968600 \\ \text { O } & 0.50088900 & -2.84560500 & 0.20856400 \\ \text { H } & -1.90792100 & -2.18088100 & 0.08780700 \\ \text { O } & 1.76446200 & -1.23303000 & -0.67928900 \\ \text { H } & 2.38761400 & -1.97111400 & -0.72602300 \\ \text { H } & -3.80822300 & -0.57929700 & 0.13979500 \\ \text { H } & -3.35445400 & 1.85229300 & 0.02417300 \\ \text { H } & -1.02022400 & 2.66599800 & -0.09484900 \\ \text { C } & 1.21569700 & 1.32925200 & -0.01599600 \\ \text { O } & 1.52555100 & 2.15141900 & -0.83918000 \\ \text { C } & 2.10382600 & 1.01103400 & 1.16219700 \\ \text { H } & 1.88806400 & 0.04439100 & 1.60862800 \\ \text { H } & 3.14546400 & 1.07240700 & 0.85852000 \\ \text { H } & 1.91797000 & 1.77923500 & 1.91630000\end{array}$

\section{2- $-\mathrm{CF}_{3}$}

$\begin{array}{lrrr}C & -0.08116700 & -0.65604300 & 0.01688400 \\ C & -0.79068900 & 0.54786800 & 0.00599300 \\ C & -2.17986000 & 0.52038100 & 0.01363200 \\ C & -2.86471800 & -0.68223700 & 0.07688300 \\ C & -2.15848600 & -1.87287500 & 0.11143500 \\ C & -0.77219100 & -1.85786000 & 0.06606300\end{array}$




$\begin{array}{lrrr}H & -3.94545700 & -0.68677400 & 0.09444100 \\ \mathrm{C} & -0.17908600 & 1.91383200 & -0.01220900 \\ \mathrm{O} & -0.64460300 & 2.83883800 & -0.61767300 \\ \mathrm{H} & -2.68164000 & -2.81763900 & 0.15672000 \\ \mathrm{H} & -2.71018400 & 1.46129100 & -0.02720500 \\ \mathrm{O} & 0.89854400 & 2.01972600 & 0.77378000 \\ \mathrm{H} & 1.23258800 & 2.92369000 & 0.69017800 \\ \mathrm{H} & -0.22154500 & -2.78642800 & 0.05368400 \\ \mathrm{C} & 1.42640400 & -0.71338900 & -0.08289000 \\ \mathrm{~F} & 2.03020900 & -0.49654300 & 1.08753000 \\ \mathrm{~F} & 1.83416000 & -1.91964700 & -0.50245000 \\ \mathrm{~F} & 1.90157200 & 0.17611100 & -0.96190500\end{array}$

\begin{tabular}{|c|c|c|c|}
\hline \multicolumn{4}{|c|}{$2-\mathrm{C}\left(\mathrm{CF}_{3}\right)_{3}$} \\
\hline C & 1.98900700 & 0.08618200 & 0.03514500 \\
\hline C & 0.74864600 & 0.74919800 & -0.08748900 \\
\hline C & 0.80802500 & 2.13352800 & -0.27193600 \\
\hline C & 1.99901600 & 2.84151300 & -0.27497800 \\
\hline C & 3.19543600 & 2.18899300 & -0.06222100 \\
\hline C & 3.17480300 & 0.81614400 & 0.08577200 \\
\hline $\mathrm{H}$ & -0.08313000 & 2.71369400 & -0.41726200 \\
\hline $\mathrm{H}$ & 1.97043700 & 3.91117100 & -0.42723500 \\
\hline $\mathrm{H}$ & 4.13060000 & 2.72944300 & -0.02868100 \\
\hline $\mathrm{H}$ & 4.09430900 & 0.26593300 & 0.22583600 \\
\hline C & 2.28405000 & -1.38509900 & 0.01940300 \\
\hline $\mathrm{O}$ & 3.02728500 & -1.93223400 & 0.78262500 \\
\hline $\mathrm{O}$ & 1.72980800 & -1.99708100 & -1.03239800 \\
\hline C & -0.65130000 & 0.07262400 & 0.00977600 \\
\hline C & -1.02278300 & -0.75207800 & -1.28193000 \\
\hline C & -0.76270400 & -0.85506300 & 1.25820500 \\
\hline C & -1.77864900 & 1.15127300 & 0.19459300 \\
\hline $\mathrm{H}$ & 1.96251000 & -2.93535100 & -0.98379700 \\
\hline $\mathrm{F}$ & -0.29005200 & -0.34877500 & -2.31090300 \\
\hline $\mathrm{F}$ & -0.84575400 & -2.05767000 & -1.11441700 \\
\hline $\mathrm{F}$ & -2.30341300 & -0.59522000 & -1.62002200 \\
\hline $\mathrm{F}$ & -2.94478500 & 0.60563600 & 0.51521000 \\
\hline $\mathrm{F}$ & -1.48942900 & 2.01836700 & 1.16151600 \\
\hline $\mathrm{F}$ & -0.72089700 & -0.12859000 & 2.37146800 \\
\hline $\mathrm{F}$ & -1.89941000 & -1.54536000 & 1.25779800 \\
\hline $\mathrm{F}$ & 0.22607800 & -1.73951300 & 1.32735800 \\
\hline $\mathrm{F}$ & -1.95820100 & 1.84294200 & -0.93431000 \\
\hline
\end{tabular}

$\begin{array}{lrrr}\text { 2-CN } & & & \\ \text { C } & 0.34723000 & 0.82881600 & 0.00000100 \\ \text { C } & -0.04324000 & -0.51687000 & 0.00000200 \\ \text { C } & 0.93390500 & -1.50515400 & 0.00000200 \\ \text { C } & 2.27869800 & -1.17750200 & 0.00000000 \\ \text { C } & 2.66409300 & 0.15527500 & -0.00000100 \\ \text { C } & 1.70460400 & 1.15243000 & -0.00000100\end{array}$




$\begin{array}{lrrr}\mathrm{C} & -1.45883100 & -0.99638600 & 0.00000000 \\ \mathrm{O} & -1.76258400 & -2.15818700 & -0.00000200 \\ \mathrm{H} & 0.60838000 & -2.53544600 & 0.00000200 \\ \mathrm{O} & -2.35805200 & -0.00896700 & 0.00000000 \\ \mathrm{H} & -3.23750400 & -0.41128500 & -0.00000100 \\ \mathrm{H} & 3.02394900 & -1.96025800 & 0.00000000 \\ \mathrm{H} & 3.71173700 & 0.42147500 & -0.00000300 \\ \mathrm{H} & 1.99120700 & 2.19409200 & -0.00000200 \\ \mathrm{C} & -0.56105900 & 1.94361600 & 0.00000100 \\ \mathrm{~N} & -1.18929800 & 2.90332900 & 0.00000000\end{array}$

$\begin{array}{lrrr}2-\mathrm{SO}_{2} \mathrm{Ph} & & & \\ \mathrm{C} & -1.17759600 & -0.57754500 & 0.19895600 \\ \mathrm{C} & -1.86853100 & 0.50902900 & -0.33757100 \\ \mathrm{C} & -3.05892100 & 0.27913600 & -1.01465100 \\ \mathrm{C} & -3.53387300 & -1.01171100 & -1.19221400 \\ \mathrm{C} & -2.83385900 & -2.08565800 & -0.66741200 \\ \mathrm{C} & -1.43698900 & 1.93901700 & -0.24656200 \\ \mathrm{O} & -2.17912600 & 2.84187900 & 0.01279800 \\ \mathrm{H} & -3.60537000 & 1.12956600 & -1.39804500 \\ \mathrm{O} & -0.15255500 & 2.11175200 & -0.58716100 \\ \mathrm{H} & 0.05132800 & 3.05105300 & -0.47557900 \\ \mathrm{H} & -4.45687300 & -1.17397200 & -1.73102700 \\ \mathrm{H} & -3.20661600 & -3.09298800 & -0.78874600 \\ \mathrm{C} & -1.66386600 & -1.86711000 & 0.04620700 \\ \mathrm{H} & -1.13824400 & -2.68347100 & 0.52052700 \\ \mathrm{~S} & 0.25185600 & -0.39431100 & 1.27461900 \\ \mathrm{O} & 0.37836800 & -1.65596900 & 1.97928700 \\ \mathrm{O} & 0.10063600 & 0.84359400 & 2.01294200 \\ \mathrm{C} & 1.66995500 & -0.25405600 & 0.20706400 \\ \mathrm{C} & 2.47126100 & 0.86986300 & 0.30059000 \\ \mathrm{C} & 1.97652100 & -1.30649500 & -0.64157800 \\ \mathrm{C} & 3.61313000 & 0.94111900 & -0.48403600 \\ \mathrm{H} & 2.19473500 & 1.66190100 & 0.98209600 \\ \mathrm{C} & 3.11129200 & -1.21843100 & -1.43182800 \\ \mathrm{H} & 1.34238800 & -2.18263100 & -0.67533700 \\ \mathrm{C} & 3.92727100 & -0.09665400 & -1.35086200 \\ \mathrm{H} & 4.25645800 & 1.80748700 & -0.41942000 \\ \mathrm{H} & 3.36372900 & -2.02690900 & -2.10333000 \\ \mathrm{H} & 4.81542400 & -0.03413200 & -1.96458400 \\ & & & \end{array}$

\section{2-SO ${ }_{2} \mathrm{Me}$}

$\begin{array}{lrrr}\text { C } & 0.17974800 & -0.64005100 & 0.00596200 \\ \text { C } & 1.08558000 & 0.42020700 & 0.01611600 \\ \text { C } & 2.44497400 & 0.13871300 & 0.06312500 \\ \text { C } & 2.89208100 & -1.17191900 & 0.13945700 \\ \text { C } & 1.98293000 & -2.21773600 & 0.14200200 \\ \text { C } & 0.71770800 & 1.87101200 & -0.03685700 \\ \text { O } & 1.23228900 & 2.65931600 & -0.77423200\end{array}$




$\begin{array}{rrrr}H & 3.14482100 & 0.96236700 & 0.03595700 \\ \mathrm{O} & -0.17527200 & 2.21861400 & 0.90556900 \\ \mathrm{H} & -0.39227800 & 3.15068700 & 0.76114600 \\ \mathrm{H} & 3.95313100 & -1.37376100 & 0.18470000 \\ \mathrm{H} & 2.32784400 & -3.24117200 & 0.18523200 \\ \mathrm{C} & 0.62371800 & -1.95222100 & 0.05679100 \\ \mathrm{H} & -0.10070200 & -2.75198600 & -0.00568300 \\ \mathrm{~S} & -1.58216800 & -0.40181800 & -0.24257000 \\ \mathrm{O} & -2.11401500 & -1.66536500 & -0.71699500 \\ \mathrm{O} & -1.76111800 & 0.79998400 & -1.03276600 \\ \mathrm{C} & -2.24641300 & -0.13730800 & 1.38640200 \\ \mathrm{H} & -1.78422100 & 0.74413400 & 1.81657500 \\ \mathrm{H} & -3.31512800 & 0.00520500 & 1.23840500 \\ \mathrm{H} & -2.05578400 & -1.03097400 & 1.97418600\end{array}$

\begin{tabular}{|c|c|c|c|}
\hline $2-\mathrm{NO}_{2}$ & & & \\
\hline C & -0.44651000 & -0.60691300 & 0.02047500 \\
\hline C & -0.06414500 & 0.72746600 & 0.00754200 \\
\hline C & -1.06177500 & 1.69172300 & -0.00524800 \\
\hline C & -2.39903100 & 1.32210400 & -0.02629800 \\
\hline C & -2.75362700 & -0.01850600 & -0.03802700 \\
\hline C & -1.77045000 & -0.99613800 & -0.01253400 \\
\hline $\mathrm{H}$ & -3.16470800 & 2.08486300 & -0.03713200 \\
\hline C & 1.35371400 & 1.20308800 & -0.05785600 \\
\hline $\mathrm{O}$ & 1.78863400 & 2.08743100 & 0.62091800 \\
\hline $\mathrm{H}$ & -3.79469800 & -0.30751500 & -0.05426300 \\
\hline $\mathrm{H}$ & -0.77199300 & 2.73320500 & -0.00618700 \\
\hline $\mathrm{O}$ & 2.04366600 & 0.59672400 & -1.03192400 \\
\hline $\mathrm{H}$ & 2.95192000 & 0.92847400 & -1.00085700 \\
\hline $\mathrm{H}$ & -2.01016100 & -2.04884200 & 0.00035800 \\
\hline $\mathrm{N}$ & 0.56691200 & -1.66820200 & 0.16914300 \\
\hline $\mathrm{O}$ & 0.32653100 & -2.74037600 & -0.33622200 \\
\hline $\mathrm{O}$ & 1.55019400 & -1.39999400 & 0.82044800 \\
\hline
\end{tabular}

\begin{tabular}{|c|c|c|c|}
\hline \multicolumn{4}{|c|}{$2-\mathrm{COCF}_{3}$} \\
\hline C & -0.58312100 & -0.67123400 & -0.30709200 \\
\hline C & -1.23975500 & 0.53855200 & -0.06700600 \\
\hline C & -2.58308600 & 0.53911700 & 0.28016000 \\
\hline C & -3.27888800 & -0.65533800 & 0.38349200 \\
\hline C & -2.63450900 & -1.85573800 & 0.12482000 \\
\hline C & -1.29126600 & -1.86388600 & -0.22215900 \\
\hline C & -0.57833800 & 1.86085300 & -0.23894400 \\
\hline $\mathrm{O}$ & -0.99941300 & 2.89947800 & 0.18289900 \\
\hline $\mathrm{H}$ & -3.06773200 & 1.48975000 & 0.45351500 \\
\hline $\mathrm{O}$ & 0.55075900 & 1.76809600 & -0.96763600 \\
\hline $\mathrm{H}$ & 0.95754500 & 2.64477700 & -1.00718400 \\
\hline $\mathrm{H}$ & -4.32507500 & -0.64765100 & 0.65491000 \\
\hline $\mathrm{H}$ & -3.17539600 & -2.78925000 & 0.19330300 \\
\hline C & 0.87272500 & -0.79772500 & -0.62392100 \\
\hline
\end{tabular}




$\begin{array}{lrrr}\text { O } & 1.29795900 & -1.40590100 & -1.56016000 \\ \text { H } & -0.77784200 & -2.79437200 & -0.42184600 \\ \text { C } & 1.90392200 & -0.29776600 & 0.42498600 \\ \text { F } & 1.37020000 & 0.50487400 & 1.34808900 \\ F & 2.91790100 & 0.33313500 & -0.14791400 \\ F & 2.38611700 & -1.36885900 & 1.06209900\end{array}$

\begin{tabular}{|c|c|c|c|}
\hline \multicolumn{4}{|c|}{$2-\mathrm{SO}_{2} \mathrm{~F}$} \\
\hline C & -0.12582900 & -0.60743400 & 0.05661400 \\
\hline C & -1.11168700 & 0.37767300 & -0.00250100 \\
\hline C & -2.44316600 & -0.01283000 & -0.03107100 \\
\hline C & -2.78829500 & -1.35325300 & 0.04178300 \\
\hline C & -1.79942400 & -2.31918100 & 0.13452000 \\
\hline C & -0.85023000 & 1.85180100 & -0.00063100 \\
\hline $\mathrm{O}$ & -1.49888100 & 2.64288900 & -0.62182600 \\
\hline $\mathrm{H}$ & -3.19877200 & 0.75687200 & -0.10308600 \\
\hline $\mathrm{O}$ & 0.13934000 & 2.18106100 & 0.83579400 \\
\hline $\mathrm{H}$ & 0.30951200 & 3.12973900 & 0.75060100 \\
\hline $\mathrm{H}$ & -3.82985800 & -1.64141100 & 0.02741600 \\
\hline $\mathrm{H}$ & -2.06129600 & -3.36595800 & 0.19422200 \\
\hline C & -0.46215300 & -1.95028500 & 0.12945100 \\
\hline $\mathrm{H}$ & 0.31065600 & -2.70271200 & 0.16484800 \\
\hline S & 1.59938200 & -0.17129900 & -0.03231000 \\
\hline $\mathrm{O}$ & 2.19999200 & -0.10468100 & 1.25907300 \\
\hline $\mathrm{O}$ & 1.81133500 & 0.82494600 & -1.03224200 \\
\hline & 2.12778300 & -1.52315600 & -0.66804700 \\
\hline
\end{tabular}

$\begin{array}{lrrr}2-\mathrm{SO}_{2} \mathrm{CF}_{3} & & & \\ \mathrm{C} & -0.73646400 & -0.63677000 & 0.17635800 \\ \mathrm{C} & -1.58930900 & 0.45003100 & -0.02216400 \\ \mathrm{C} & -2.91151200 & 0.20437000 & -0.36010500 \\ \mathrm{C} & -3.36500400 & -1.09555600 & -0.53612900 \\ \mathrm{C} & -2.50286400 & -2.16457500 & -0.35693300 \\ \mathrm{C} & -1.18176000 & 1.88545400 & 0.08408500 \\ \mathrm{O} & -1.86619700 & 2.73946900 & 0.56660400 \\ \mathrm{H} & -3.57458500 & 1.04879100 & -0.48651300 \\ \mathrm{O} & 0.00214900 & 2.11194800 & -0.49792100 \\ \mathrm{H} & 0.25349500 & 3.03122300 & -0.32952000 \\ \mathrm{H} & -4.39616600 & -1.27000100 & -0.80957900 \\ \mathrm{H} & -2.85417200 & -3.17827200 & -0.48663900 \\ \mathrm{C} & -1.18544400 & -1.93788400 & 0.01727700 \\ \mathrm{H} & -0.51034000 & -2.75784900 & 0.21429200 \\ \mathrm{~S} & 0.91133500 & -0.46267500 & 0.85103100 \\ \mathrm{O} & 1.35016600 & -1.77153900 & 1.26994000 \\ \mathrm{O} & 0.95578900 & 0.66556800 & 1.74589000 \\ \mathrm{C} & 2.01788800 & -0.05561200 & -0.63815300 \\ \mathrm{~F} & 1.31292600 & -0.08162800 & -1.75879700 \\ \mathrm{~F} & 2.57665400 & 1.12734900 & -0.48501400 \\ \mathrm{~F} & 2.96508200 & -0.97143300 & -0.70952900\end{array}$




$\begin{array}{lrrr}2-\mathrm{SO}_{2} \mathrm{Cl} & & & \\ \mathrm{C} & 0.13407800 & 0.55743500 & -0.16545300 \\ \mathrm{C} & 1.38393100 & -0.00778400 & 0.07464700 \\ \mathrm{C} & 2.48090100 & 0.83688900 & 0.18716300 \\ \mathrm{C} & 2.33951300 & 2.20429900 & 0.01376300 \\ \mathrm{C} & 1.09503800 & 2.74451900 & -0.27341300 \\ \mathrm{C} & 1.66059000 & -1.47903800 & 0.15630100 \\ \mathrm{O} & 2.32281300 & -1.98748700 & 1.01241300 \\ \mathrm{H} & 3.44590300 & 0.39880400 & 0.40115900 \\ \mathrm{O} & 1.16197300 & -2.12872300 & -0.90181800 \\ \mathrm{H} & 1.31494300 & -3.07568800 & -0.77375100 \\ \mathrm{H} & 3.20392300 & 2.84769500 & 0.09777000 \\ \mathrm{H} & 0.98136300 & 3.80910700 & -0.41952900 \\ \mathrm{C} & -0.01713900 & 1.92134500 & -0.35163800 \\ \mathrm{H} & -0.99606800 & 2.33843900 & -0.53734000 \\ \mathrm{~S} & -1.31825900 & -0.48965600 & -0.20903800 \\ \mathrm{O} & -1.11577200 & -1.62613900 & 0.64236600 \\ \mathrm{O} & -1.79450300 & -0.62471500 & -1.55386100 \\ \mathrm{Cl} & -2.70091000 & 0.69333200 & 0.77266300\end{array}$

\section{References}

1. (a) Dai, J. J., et al., Chem. Commun. 2011, 47, 677; (b) Werner, E. W.; Mei, T. S.; Burckle, A. J.; Sigman, M. S., Science 2012, 338, 1455.

2. Mei, T. S.; Werner, E. W.; Burckle, A. J.; Sigman, M. S., J. Am. Chem. Soc. 2013, 135, 6830.

3. Frisch, M. J. T., G. W.; Schlegel, H. B.; Scuseria, G. E.; Robb, M. A.; Cheeseman, J. R.; Scalmani, G.; Barone, V.; Mennucci, B.; Petersson, G. A.; Nakatsuji, H.; Caricato, M.; Li, X.; Hratchian, H. P.; Izmaylov, A. F.; Bloino, J.; Zheng, G.; Sonnenberg, J. L.; Hada, M.; Ehara, M.; Toyota, K.; Fukuda, R.; Hasegawa, J.; Ishida, M.; Nakajima, T.; Honda, Y.; Kitao, O.; Nakai, H.; Vreven, T.; Montgomery, J. A., Jr.; Peralta, J. E.; Ogliaro, F.; Bearpark, M.; Heyd, J. J.; Brothers, E.; Kudin, K. N.; Staroverov, V. N.; Kobayashi, R.; Normand, J.; Raghavachari, K.; Rendell, A.; Burant, J. C.; Iyengar, S. S.; Tomasi, J.; Cossi, M.; Rega, N.; Millam, M. J.; Klene, M.; Knox, J. E.; Cross, J. B.; Bakken, V.; Adamo, C.; Jaramillo, J.; Gomperts, R.; Stratmann, R. E.; Yazyev, O.; Austin, A. J.; Cammi, R.; Pomelli, C.; Ochterski, J. W.; Martin, R. L.; Morokuma, K.; Zakrzewski, V. G.; Voth, G. A.; Salvador, P.; Dannenberg, J. J.; Dapprich, S.; Daniels, A. D.; Farkas, Ö.; Foresman, J. B.; Ortiz, J. V.; Cioslowski, J.; Fox, D. J. Gaussian 09, Gaussian, Inc.: Wallingford CT, 2009.

4. $\quad$ Schäfer, A.; Huber, C.; Ahlrichs, R., J. Chem. Phys. 1994, 100, 5829.

5. $\quad$ Quinn, J. A. Molecular Modeling Pro, ChemSW, Inc.: Fairfield, CA.

6. Hansch, C.; Leo, A.; Taft, R. W., Chem. Rev. 1991, 91, 165.

7. MATLAB Student Version, The MathWorks, Inc.: Natick, MA, 2014.

8. Milo, A.; Bess, E. N.; Sigman, M. S., Nature 2014, 507, 210.

9. Seeman, J. I., J. Chem. Edu. 1986, 63, 42. 


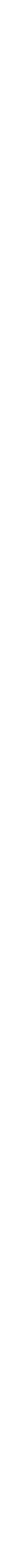


<smiles>CCC(CCC=O)c1ccccc1C(F)(F)F</smiles>

\begin{tabular}{|c|c|c|c|c|c|}
\hline 1 & $T$ & $T$ & $T$ & $T$ & \\
\hline 40 & 20 & 10 & 0 & -10 & -2 \\
\hline
\end{tabular}


CBS-01-060A-c2-R8-9-1H
CBS-01-060A-c2-R8-9

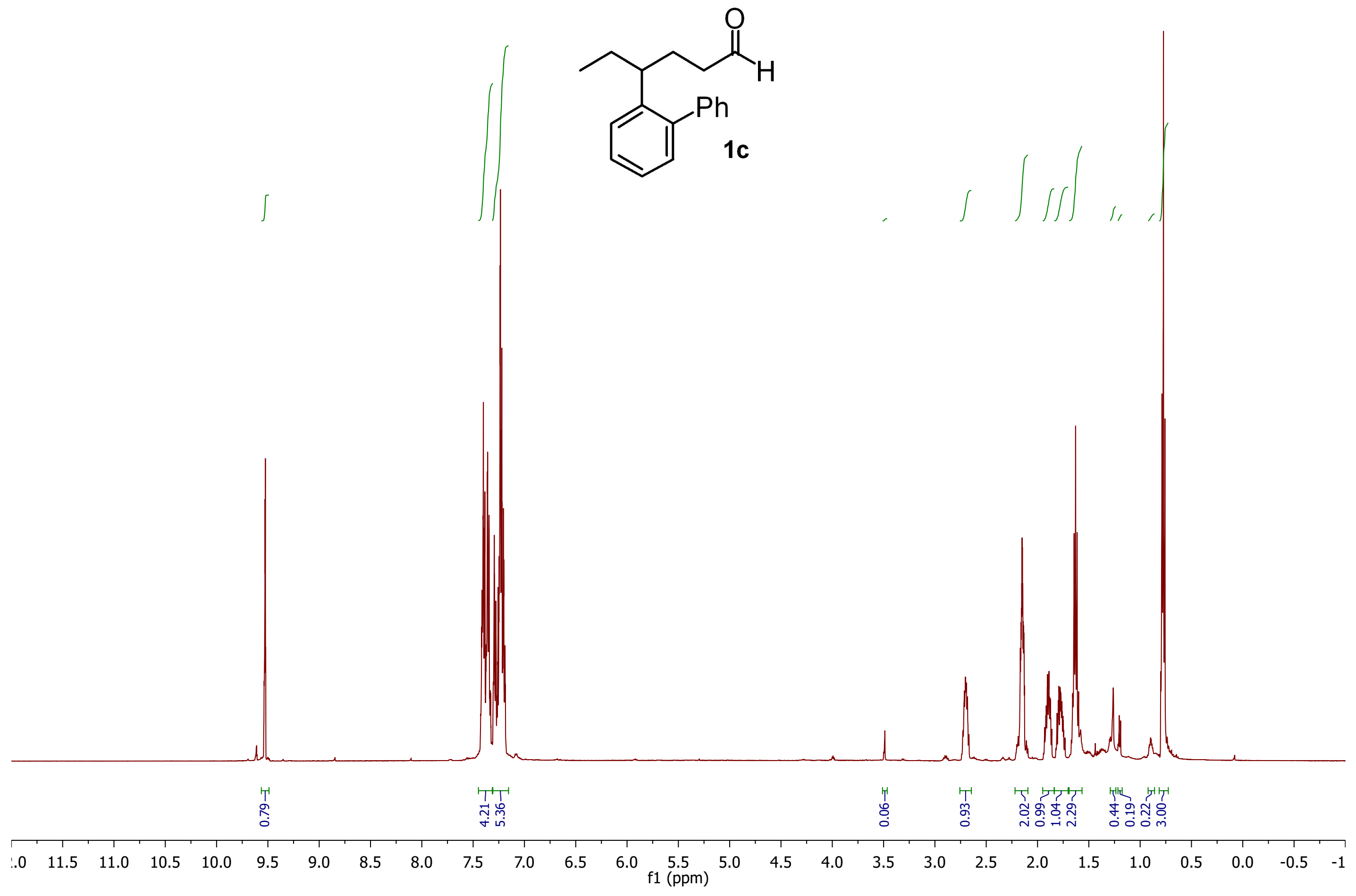




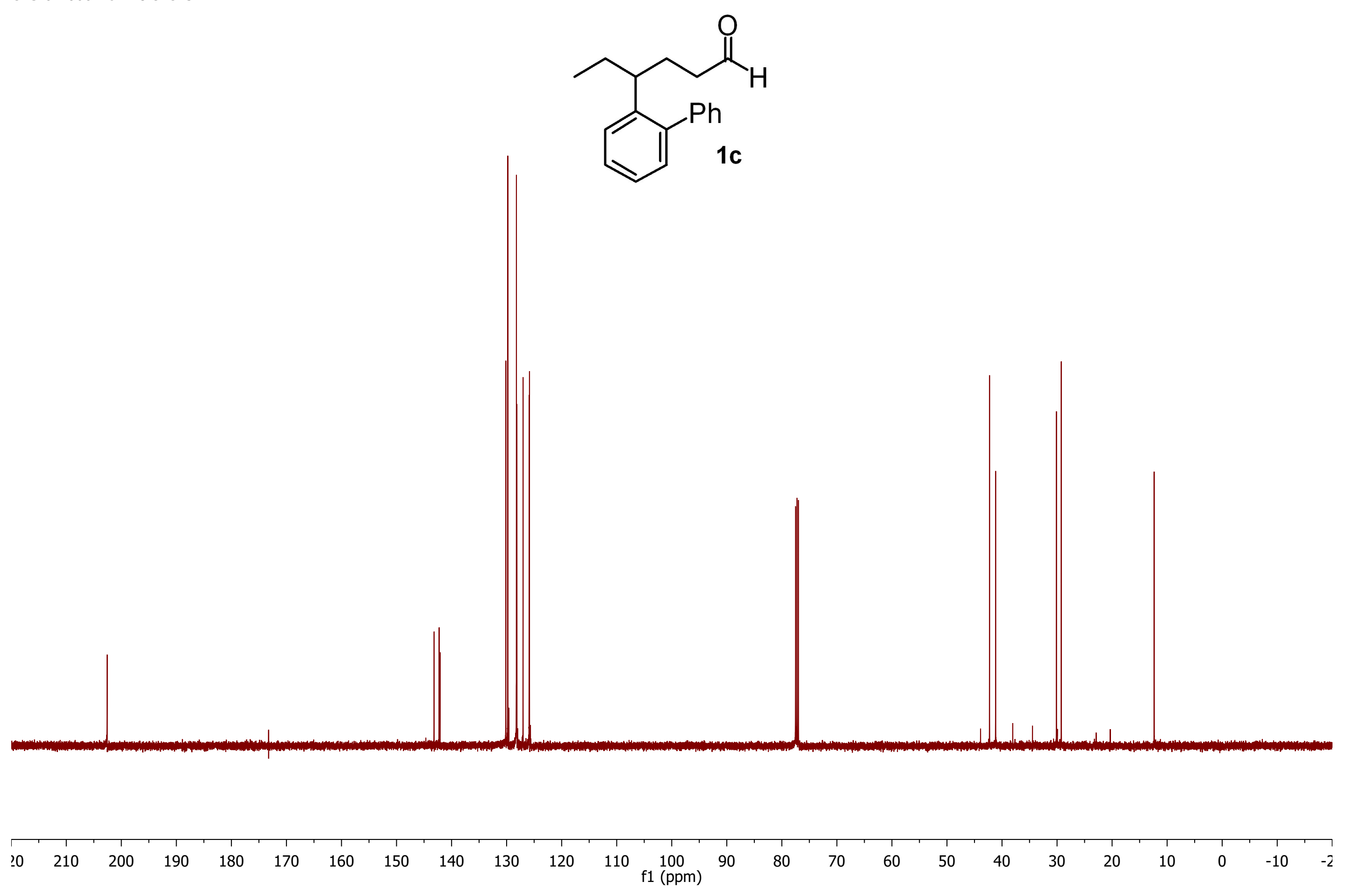




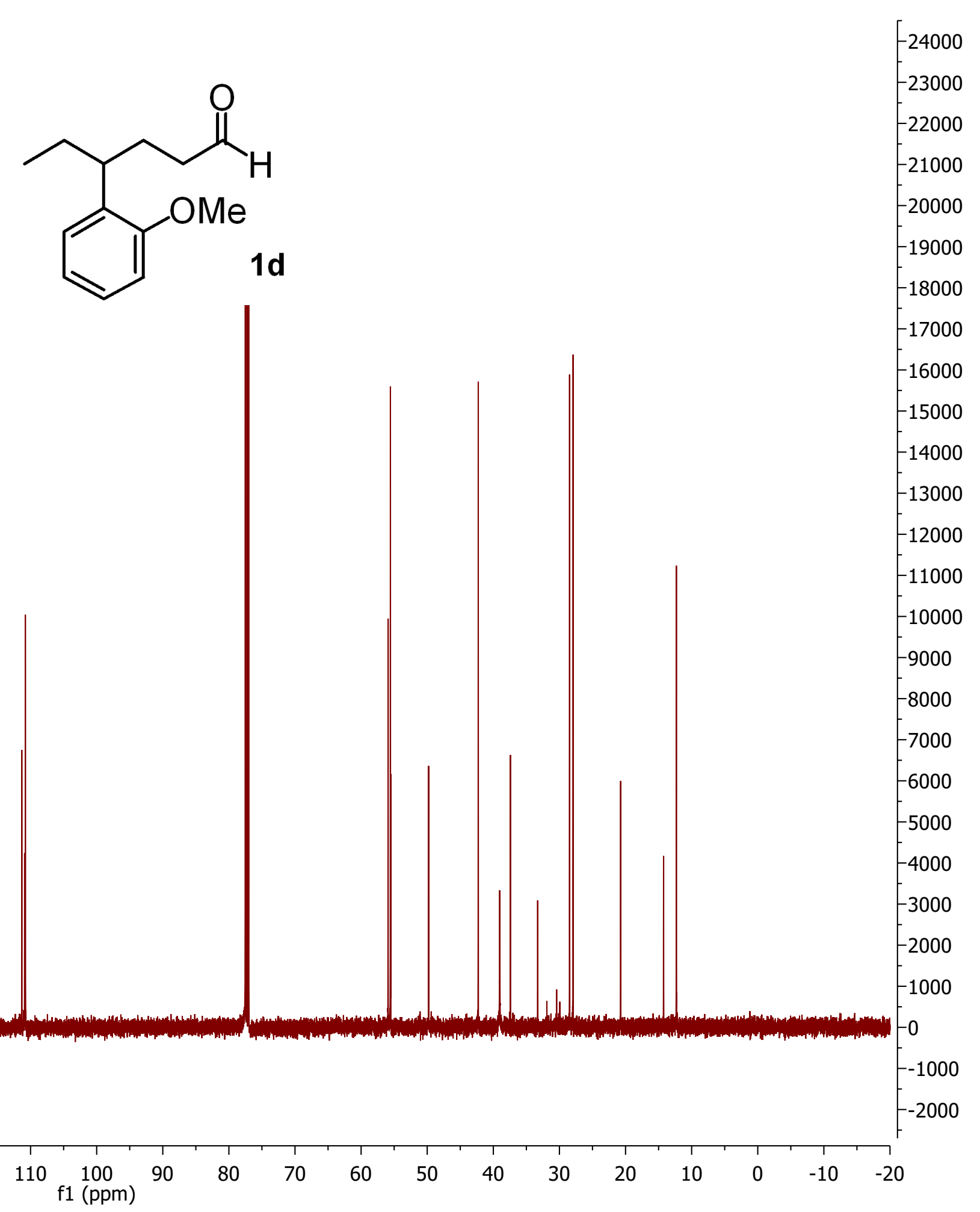



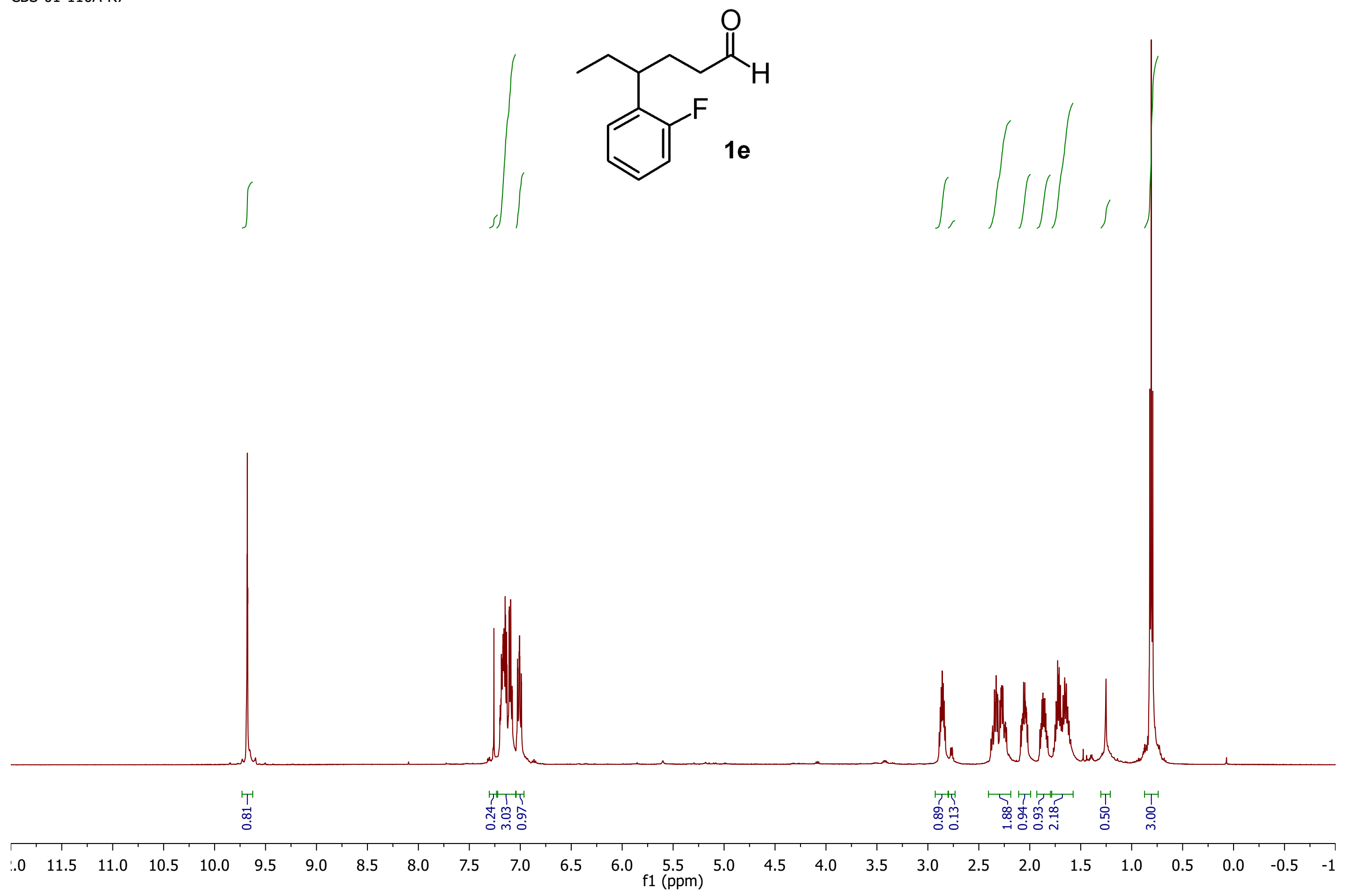
<smiles>CCC(CCC=O)c1ccccc1F</smiles>

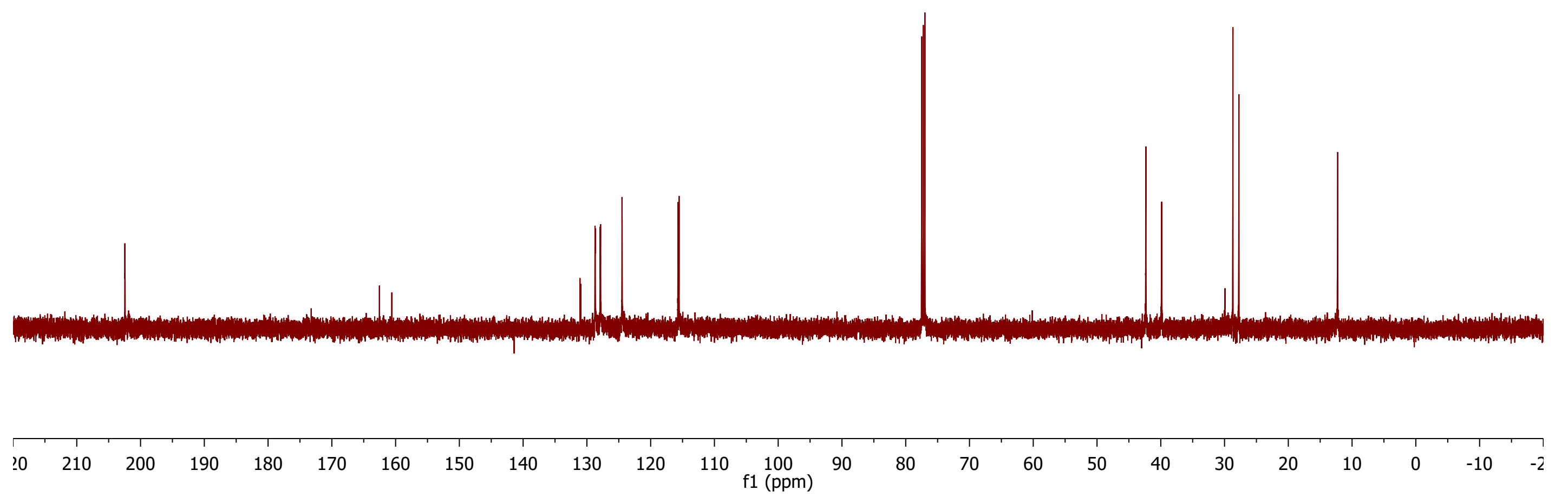



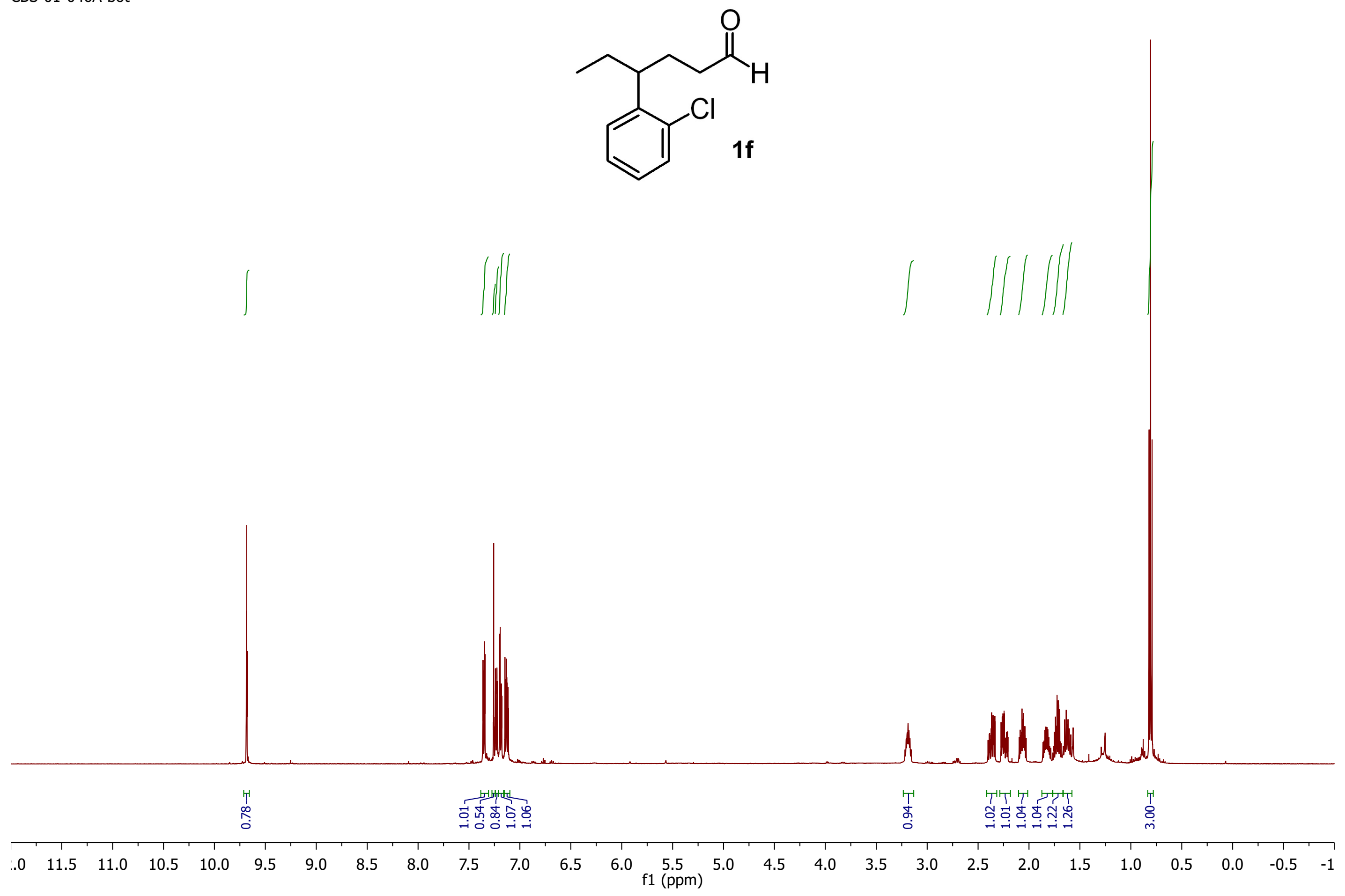


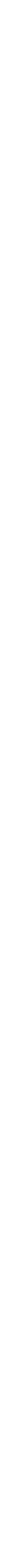

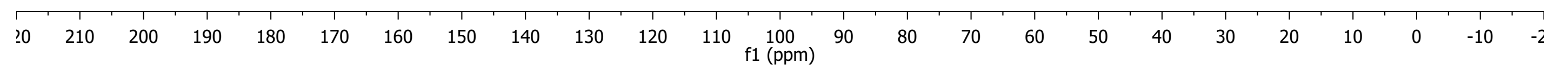



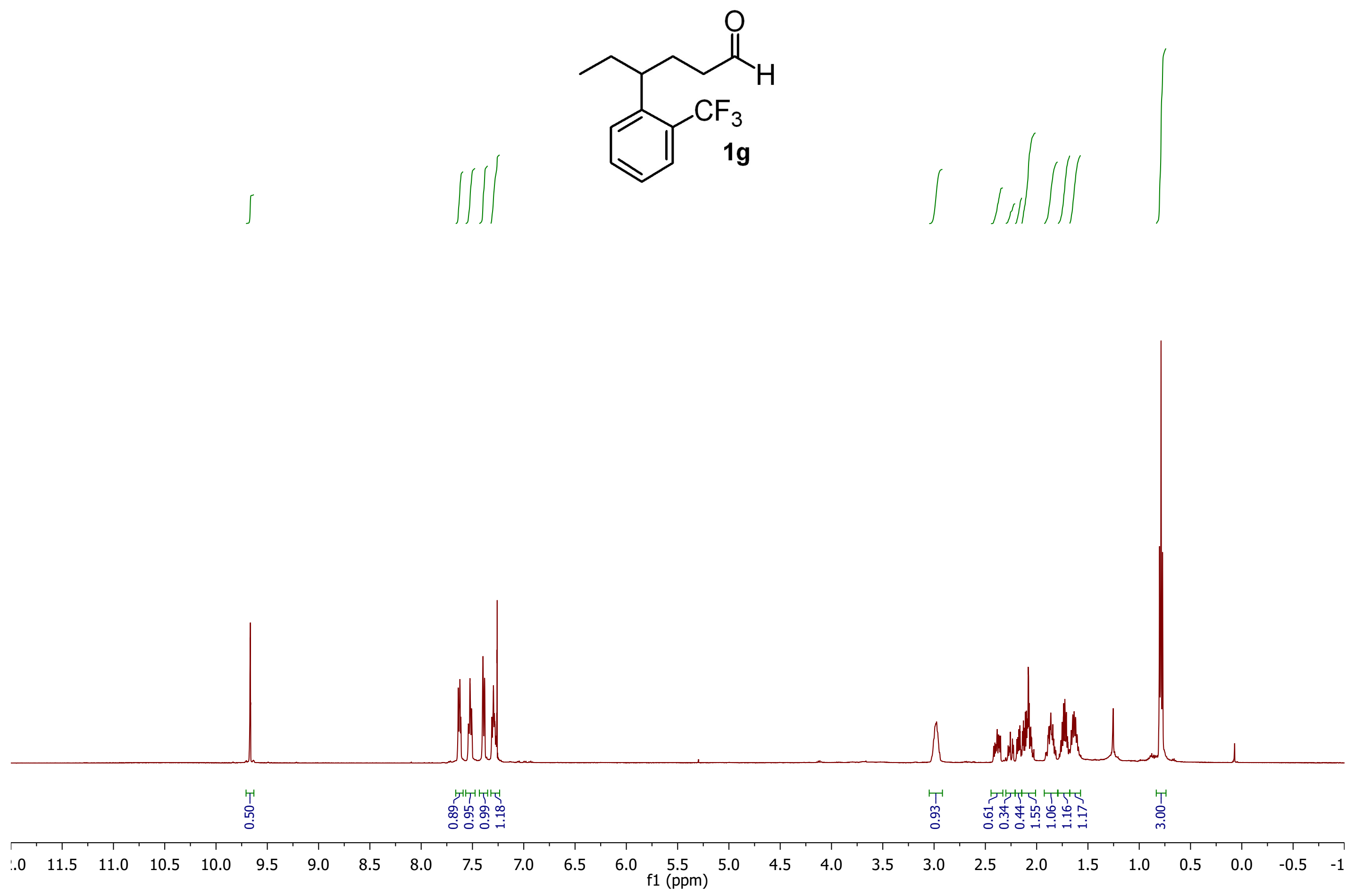


$$
\mathrm{H}
$$

